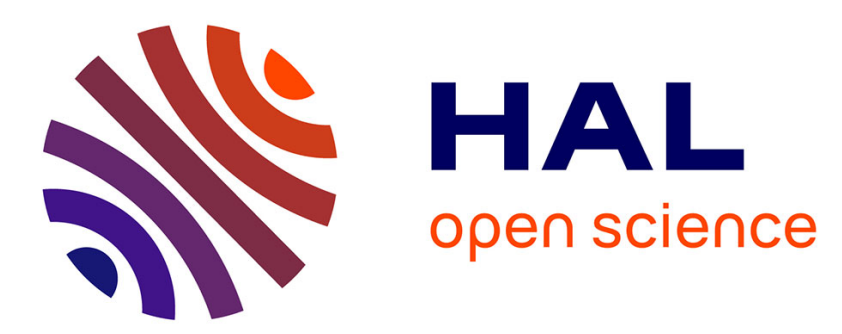

\title{
Review of Extractive Distillation. Process design, operation optimization and control
}

Vincent Gerbaud, Ivonne Rodríguez-Donis, Laszlo Hegely, Péter Láng, Ferenc Dénes, Xinqiang You

\section{- To cite this version:}

Vincent Gerbaud, Ivonne Rodríguez-Donis, Laszlo Hegely, Péter Láng, Ferenc Dénes, et al.. Review of Extractive Distillation. Process design, operation optimization and control. Chemical Engineering Research and Design, 2019, 141, pp.229-271. 10.1016/j.cherd.2018.09.020 . hal-02161920

\section{HAL Id: hal-02161920 \\ https://hal.science/hal-02161920}

Submitted on 21 Jun 2019

HAL is a multi-disciplinary open access archive for the deposit and dissemination of scientific research documents, whether they are published or not. The documents may come from teaching and research institutions in France or abroad, or from public or private research centers.
L'archive ouverte pluridisciplinaire HAL, est destinée au dépôt et à la diffusion de documents scientifiques de niveau recherche, publiés ou non, émanant des établissements d'enseignement et de recherche français ou étrangers, des laboratoires publics ou privés. 


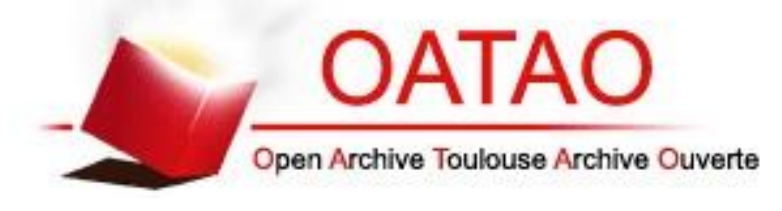

Open Archive Toulouse Archive Ouverte

OATAO is an open access repository that collects the work of Toulouse researchers and makes it freely available over the web where possible

This is an author's version published in: http://oatao.univ-toulouse.fr/239894

Official URL: https://doi.org/10.1016/j.cherd.2018.09.020

\section{To cite this version:}

Gerbaud, Vincent" and Rodríguez-Donis, Ivonne $\leftrightarrows$ and Hegely, Laszlo and Láng, Péter and Dénes, Ferenc and You, Xinqiang Review of Extractive Distillation. Process design, operation optimization and control. (2018) Chemical Engineering Research and Design, 141. 1-43. ISSN 0263-8762

Any correspondence concerning this service should be sent to the repository administrator: tech-oatao@listes-diff.inp-toulouse.fr 


\title{
Review of extractive distillation. Process design, operation, optimization and control
}

\author{
Vincent Gerbaud ${ }^{a, *}$, Ivonne Rodriguez-Donis ${ }^{b}$, Laszlo Hegely ${ }^{c}$, Peter Lang ${ }^{c}$, \\ Ferenc Denes ${ }^{d}$, XinQiang You ${ }^{e}$ \\ a Laboratoire de Génie Chimique, Université de Toulouse, CNRS, Toulouse, France \\ ${ }^{\mathrm{b}}$ Laboratoire de Chimie Agro-industrielle, LCA, Université de Toulouse, INRA, Toulouse, France \\ c Department of Building Services and Process Engineering, Budapest University of Technology and Economics, \\ Muegyetem rkp. 3-9, H-1111 Budapest, Hungary \\ d Department of Energy Engineering, Budapest University of Technology and Economics, Muegyetem rkp. 3-9, \\ H-1111 Budapest, Hungary \\ e Fujian Universities Engineering Research Center of Reactive Distillation Technology, College of Chemical \\ Engineering, Fuzhou University, Fuzhou 350116, Fujian, China
}

Keywords:

Batch extractive distillation

Continuous extractive distillation

Entrainer

Extractive column configuration

Heterogeneous extractive

distillation

Operation and control

\begin{abstract}
Extractive distillation processes enable the separation of non-ideal mixtures, including minimum or maximum boiling azeotropes and low relative volatility mixtures. Unlike azeotropic distillation, the entrainer fed at another location than the main mixture induces an extractive section within the column. A general feasibility criterion shows that intermediate and light entrainers and heterogeneous entrainers are suitable along common heavy entrainers. Entrainer selection rules rely upon selectivity ratios and residue curve map $(\mathrm{rcm})$ topology including univolatility curves. For each type of entrainer, we define extractive separation classes that summarize feasibility regions, achievable products and entrainer - feed flow rate ratio limits. Case studies are listed as Supplementary materials. Depending on the separation class, a direct or an indirect split column configuration will allow to obtain a distillate product or a bottom product, which is usually a saddle point of $\mathrm{rcm}$. Batch and continuous process operations differ mainly by the feasible ranges for the entrainer - feed flow rate ratio and reflux ratio. The batch process is feasible under total reflux and can orient the still path by changing the reflux policy. Optimisation of the extractive process must systematically consider the extractive column along with the entrainer regeneration column that requires energy and may limit the product purity in the extractive column through recycle. For the sake of reducing the energy cost and the total cost, pressure change can be beneficial as it affects volatility, or new process structures can be devised, namely heat integrated extractive distillation, extractive divided wall column or processes with preconcentrator.
\end{abstract}

\section{Contents}

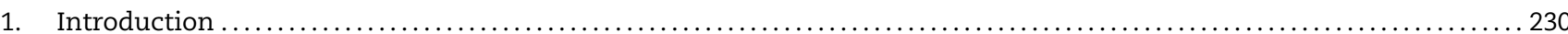

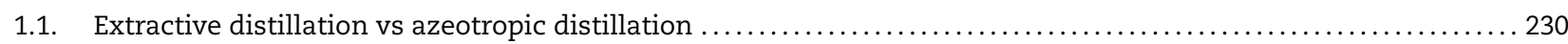

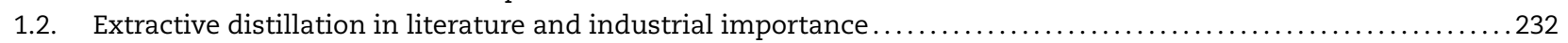

\footnotetext{
* Corresponding author.

E-mail address: Vincent.Gerbaud@ensiacet.fr (V. Gerbaud). https://doi.org/10.1016/j.cherd.2018.09.020
} 


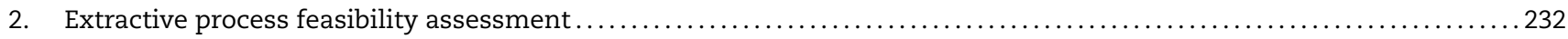

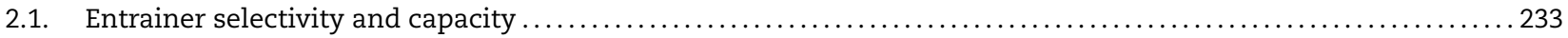

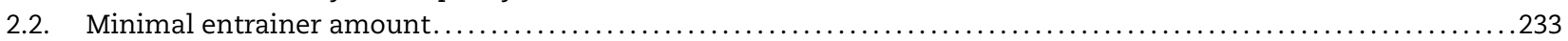

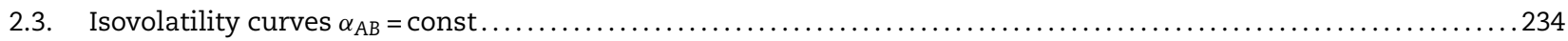

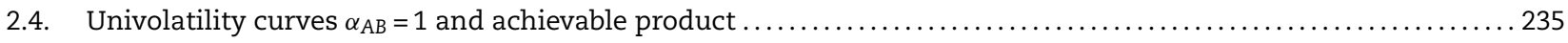

2.5. General feasibility criterion for extractive distillation with an intermediate feed location $\ldots \ldots \ldots \ldots \ldots \ldots \ldots 237$

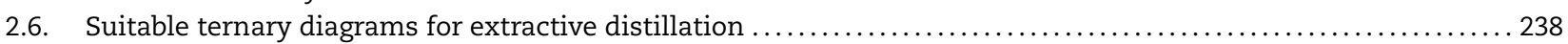

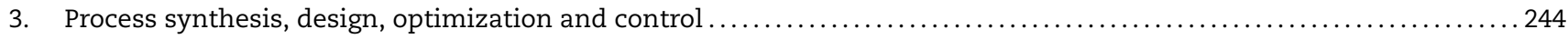

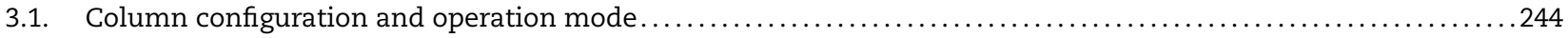

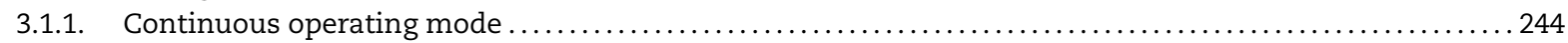

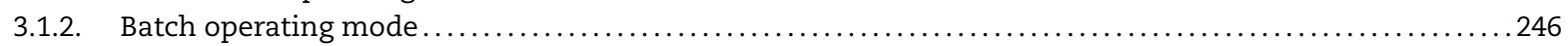

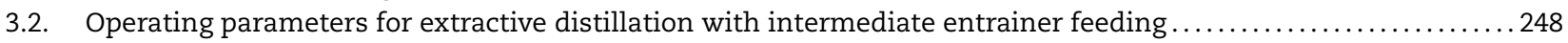

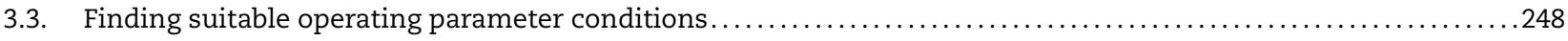

3.3.1. Defining suitable product recovery yields and recycle entrainer purity $\ldots \ldots \ldots \ldots \ldots \ldots \ldots \ldots \ldots \ldots 248$

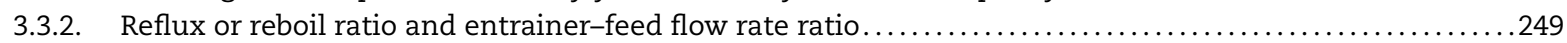

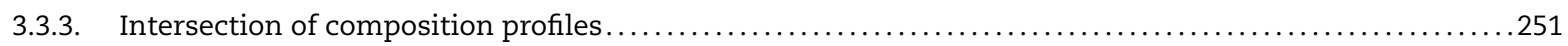

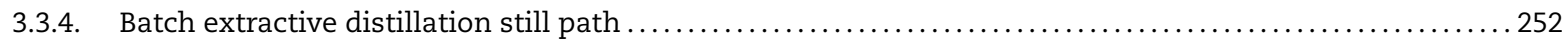

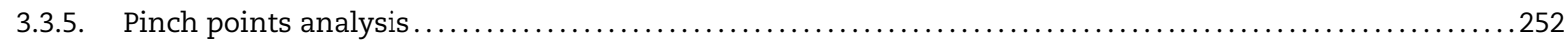

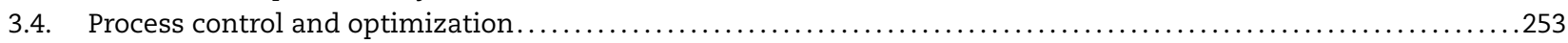

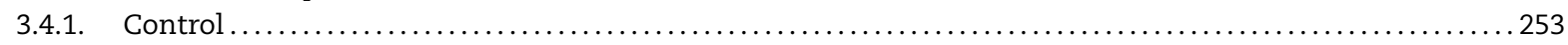

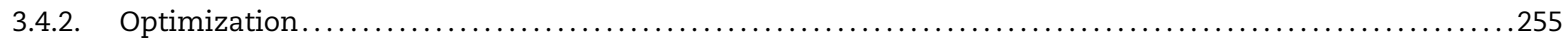

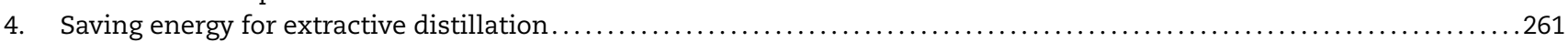

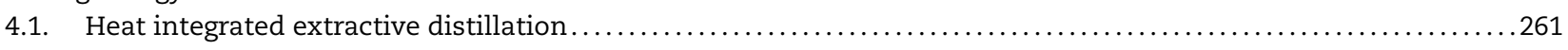

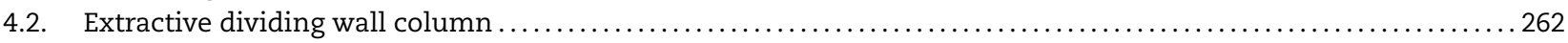

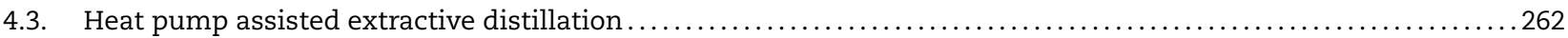

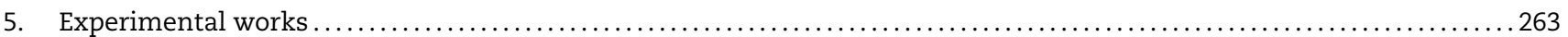

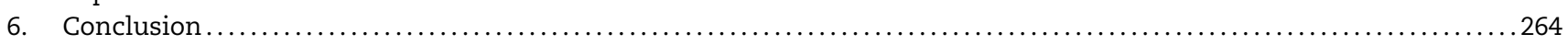

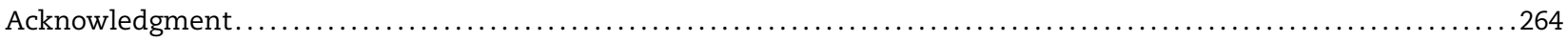

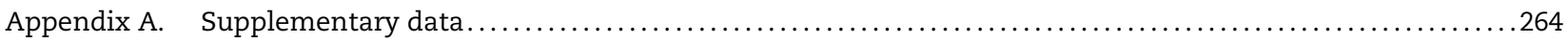

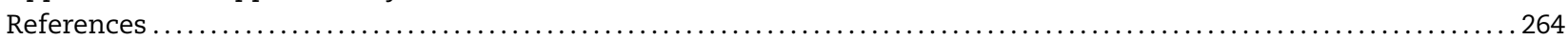

\section{Introduction}

Azeotropes are mixtures of specific composition with a boiling temperature at which the liquid and the vapour compositions are equal under a fixed pressure. The boiling temperature can be lower or greater than the boiling temperature of the original mixture compounds. This impedes the recovery of each mixture components by simple distillation, since for example a minimum boiling azeotrope will be distilled out first. To overcome that, many distillation processes have been devised. Some require the addition of a third body compound, so-called solvent or entrainer, like azeotropic distillation extractive distillation or reactive distillation (Segovia-Hernández et al., 2015), some exploit the change of azeotrope composition and boiling temperature with the pressure, like pressure-swing distillation (Liang et al., 2017), and some like pervaporation combine distillation with partial vaporization into a selective membrane (Liu et al., 2017).

After Section 1, giving an overview of the literature over the years and of the essential differences between extractive and azeotropic distillation, we have organized this review paper to address the questions following an engineer's thinking: Section 2. Will extractive distillation be suitable for separating mixture components and how can I select an entrainer? Section 3. How can I carry out the process synthesis and design? Should I expect limiting values for reflux ratio or for entrainer feed ratio? Are there differences between batch and continuous mode? What can I optimize and control, and how? Section 4. Knowing that distillation requires a lot of energy, what are the options for saving energy?

\subsection{Extractive distillation us azeotropic distillation}

Both requiring the addition of an entrainer, azeotropic and extractive distillations are among the leading processes for the separation of minimum (minT) or maximum (maxT) boiling azeotropic mixtures or low relative volatility mixtures (low $\alpha$ ). For many years, extractive distillation was considered as a special case of azeotropic distillation in a double-feed column, deemed suitable for the separation of minimum boiling azeotropes by using a heavy entrainer that would not form any new azeotrope (Benedict and Rubbin, 1945). But, since the 90's (Laroche et al., 1991) the two processes are considered as distinct since they obey different feasibility rules and operate in different column configurations. Below we consider the separation of a binary mixture A-B, A being more volatile than B, with an entrainer $E$ that is either a heavy ( $E$ boiling temperature greater than $\mathrm{A}$ and $\mathrm{B})$, low, or intermediate boiler. For multicomponent mixtures, it is customary to identify the key binary mixture and then run a preliminary process analysis, design and synthesis with methods and tools suitable for ternary mixtures. The whole mixture is then only considered at the simulation step. Only a few works deal with the extractive distillation of more than three and up to seven component mixtures with multiple azeotropes, either in batch (Lang et al., 2006, 2010, 2012; Hegely et al., 2013; Hegely and Lang, 2014, 2015), or in continuous operation mode (Berg, 1983; Lei et al., 2002; Modla and Lang, 2012; Raeva and Sazonova, 2015; Timoshenko et al., 2015; Luyben, 2016c; Wang et al., 2016, 2018a,b; Moraru and Bildea, 2017; Gao et al., 2017; Zhao et al., 2017a; Gu et al., 2018b; Yang et al., 2018). They often deal with mixtures displaying several azeotropes, like methyl acetate-methanol-water, tetrahydrofuran-methanol-water, tetrahydrofuran-ethanol-water, or with an aromatic mixture benzene-cyclohexane-toluene.

In azeotropic distillation, an entrainer feed $F_{E}$ enters the azeotropic column along with the main feed $F_{A B}$, defining two sections, rectifying and stripping ones. The feasibility of the azeotropic distillation process relies on the analysis of ternary residue curve maps A-B-E as the liquid composition profile in a packed column with an infinite number of trays and operating under total reflux ratio is a residue curve (Widagdo and Seider, 1996; Laroche et al., 1992a; Wahnschafft and Westerberg, 1993; Gerbaud et al., 2006). Residue curves properties are summarized best in Kiva et al. (2003). They are computed by the following differen- 


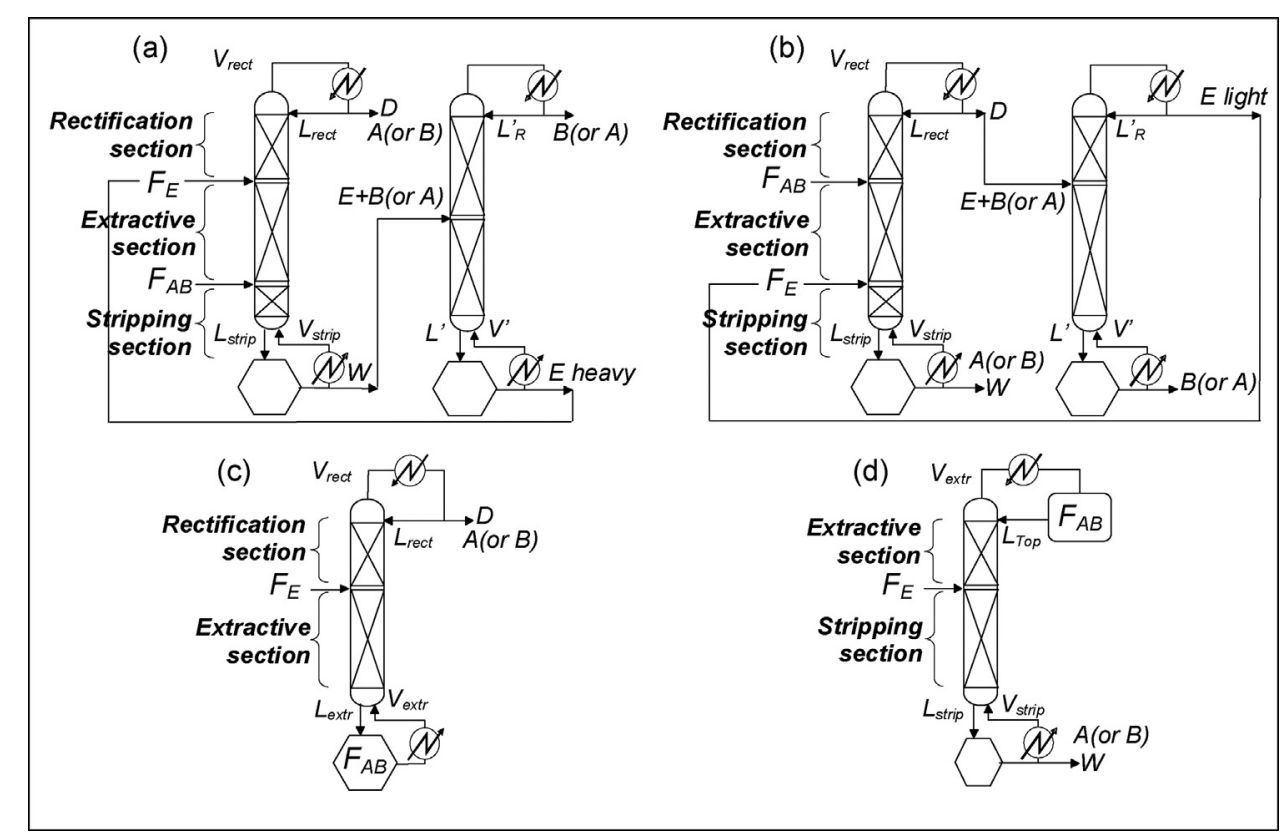

Fig. 1 - Typical flowsheets for the extractive distillation process. (a) Continuous direct split with a heavy entrainer (b) continuous indirect split with a light entrainer. (c) Batch direct split with a heavy entrainer (d) batch indirect split with a light entrainer.

tial equation, highlighting the composition change with the distillation driving force $\left(x_{i}-y_{i}\right)$ over an infinite packing height $h$ :

$\frac{d x_{i}}{d h}=x_{i}-y_{i}^{*} \quad i=1, n c$

where $y_{i}^{*}$ is assumed to be the vapour composition in equilibrium with the liquid composition $x_{i}$. nc is the number of components. The stationary points of the set of differential equations are pure components and azeotropes (Kiva et al., 2003). Since it is proven that residue curves are solutions to the gradient system with respect to the boiling temperature (Shcherbakova et al., 2015), the stable node is the highest boiling point of the distillation region, the unstable node is the lowest boiling point and saddle points are intermediate boiling points. Supplanting less compact classifications (Matsuyama and Nishimura, 1977; Doherty and Caldarola, 1985; Peterson and Partin, 1997), Serafimov's topological classification of 26 ternary diagrams is now used (Serafimov, 1970, 1996) and is completed for zeotropic ternary diagrams by Reshetov and Kravchenko (2007) with 33 zeotropic classes, which are distinguished by different volatility order regions. Statistical occurrences among real mixtures exist for 16 azeotropic classes (Hilmen et al., 2002; Kiva et al., 2003) and 16 zeotropic diagrams (Reshetov and Kravchenko, 2007). They are useful to identify generic distillation processes and configurations to separate ternary mixtures A-B-E and will be later used in this work to establish separation classes for extractive distillation.

For azeotropic distillation of a ternary mixture A-B-E, the possible products depend on the distillation region on the residue curve map where the composite feed $F_{T}\left(F_{E}+F_{A B}\right)$ is located (Doherty and Malone, 2001). Then, a continuous azeotropic column allows the withdrawal of the unstable node of the composite feed distillation region in the distillate (direct split) or of the stable node in the column bottom (indirect split). The entrainer should be chosen so that those possible node products are either A or B pure components. The other azeotropic column output stream is a mixed entrainer with B or A and is separated in the so-called entrainer regeneration second column from which the entrainer is recycled to the azeotropic column. A batch azeotropic column may enable recovering products in two different distillation regions if the distillation boundary is curved (Bernot et al., 1990, 1991).

In extractive distillation, the entrainer feed $F_{E}$ enters the column at a different tray than the main feed $F_{A B}$, defining an extractive section between the rectifying and the stripping sections. Fig. 1 highlights the column sections for the most typical flowsheet for the continuous process ( $\mathrm{a}$ and $\mathrm{b}$ ) combining the extractive distillation column and the entrainer regeneration column, and the batch process (c and d) where the extractive distillation and the entrainer regeneration steps are performed sequentially in the same column. Set by the univolatility curve location in the residue curve map diagram, the volatility order between the components in the column establishes the achievable product list and enables the removal of a product either in the distillate through an extractive direct split (Fig. 1a and c) or in the bottom through an extractive indirect split (Fig. $1 \mathrm{~b}$ or d). Real flowsheets include an entrainer make-up stream to compensate entrainer losses in the product streams and also heat exchangers, to perform heat integration or simply to set the entrainer recycle at the optimal temperature before entering the column, usually the temperature of the entrainer feeding tray, which could be defined in order to reduce the total entropy production (Benyounes et al., 2014).

The extractive section is an essential feature of the extractive distillation process. Its liquid composition profile behaves as a bridge connecting the rectifying and stripping section liquid composition profiles. It describes the conditions for which the entrainer concentration is large enough to alter significantly the A-B relative volatility and allow their separation. Unlike stripping and rectifying section composition profiles, it cannot be approximated by a residue curve at infinite reflux because it also depends on the entrainer-feed flow rate ratio, as described by the extractive profile equation in a later section. Thanks to the extractive section, some ternary diagrams unfeasible for azeotropic distillation are suitable for extractive distillation. Typically, an extractive distillation column allows recovering a saddle intermediate boiling point component as product, which would not be possible in azeotropic distillation, for the second most frequent 1.0-1a class ternary diagram (occurrence 21.6\%). It corresponds to the separation of a minimum boiling azeotropic mixture with a heavy entrainer, or a maximum boiling azeotropic mixture with a light entrainer. Possible products for other ternary diagram classes are discussed later and summarized in extractive separation classes' tables. A second entrainer regeneration column (or final step in batch mode) is still needed to recycle the entrainer to the extractive distillation column. The regeneration column plays a key role: it enables recycling the entrainer, but it consumes energy to do so, esp. when the entrainer is a heavy boiler. Furthermore, it sets the recycled entrainer purity, which may affect the extractive column product recovery and total energy consumption (You et al., 2016a) as described in Section 3.3.1. 
A quick literature survey on the "extractive distillation" or "azeotropic distillation" occurrence in topics (article, title, abstract, keywords) from Scopus database (https://www.elsevier.com/solutions/scopus) from 2000 to August 2016 was run. It brought out 1125 documents for extractive distillation vs 1222 for azeotropic distillation. Within the extractive distillation documents; 683 documents also refer to entrainer or solvent; 78 to the selection of entrainer; 224 to experimental data (equilibrium or process data); 314 to simulation; 271 to design; 180 to optimization and 107 to control. The topic trend goes towards optimal design with control scheme: with 25 papers over five years between 2000-2004; 77 between 2005-2010 and 158 between 2011 and August 2016. The most frequent chemicals listed in keywords refer to water (52/1125); to ethanol (156); methanol (110); and to ionic liquids (105) that we do not consider in the present review as there exists several surveys (Pereiro et al., 2012; Lei et al., 2014). Overall; more than fifty binary mixtures; either minimum or maximum boiling azeotropes or low relative volatility mixtures; have been considered by the various authors. The reader can find these mixtures and related references in Supplementary material file; ordered by extractive separation classes based on Serafimov's classification that will be presented in Section 2.6.

Only a few works consider light (32/683) or intermediate $(25 / 683)$ entrainers. By subtraction, the overwhelming number of the remaining works concern the use of a heavy entrainer for the extractive distillation process. The mixtures to be separated are rarely maximum boiling azeotropes (26/683) and low relative volatility or close boiling (67/683) mixtures. By subtraction, the studies concern overwhelmingly the separation of minimum boiling azeotropic mixtures. Entrainers are sometimes compared before selection (74/683).

The batch operation process $(113 / 1125)$ remains much less studied than the continuous process. However, its ability to drive the composition path during the operation time raised some interest in the scientific community in the 90's that marked the renewal of studies on the extractive distillation process. It started with Laroche's paper showing that not only heavy entrainers could be used, but light and intermediate boiling entrainers as well (Hunek et al., 1989; Laroche et al., 1991). Then, a systematic investigation of operating parameters and column configurations of the batch extractive process for the separation of minimum or maximum boiling azeotrope with all kinds of entrainer was conducted. It was led by several Hungarian teams (Lang et al., 1994, 1995, 1999, 2000a,b; Lelkes et al., 1998a,b, 1998c, 2002, 2003a,b; Rev et al., 2003; Modla et al., 2003; Stéger et al., 2005, 2006; Varga et al., 2006a,b) and others as well (Mujtaba, 1999; Low and Sorensen, 2002). In the mid-2000, Hungarian and French teams conducted several PhD works together (Stéger, 2006; Varga, 2006) that led to the proposition in 2009 by Cuban and French authors of a general feasibility criterion based on thermodynamic insight (Rodríguez-Donis et al., 2009a,b, 2010, 2012a,b; Shen et al., 2013; Shen and Gerbaud, 2013). Their proposal rationalized results about the extractive process feasibility from pioneering works in the 90's in continuous (Laroche et al., 1991, 1992b; Wahnschafft and Westerberg, 1993; Knapp and Doherty, 1994) and in batch (Lelkes et al., 1998a; Frits et al., 2006) that were later confirmed by Russo-German-Danish works (Kossack et al., 2008; Petlyuk et al., 1999, 2015).

Regarding design, optimization and control, the community is much larger. It was also investigated by the aforementioned authors (Lang, 1992; Lang et al., 1994, 1995; Lelkes et al., 1998a, 2002, 2003a,b; You et al., 2015a,b, 2016a,b) and several teams all over the world: American (Luyben, 2008a,b, 2013, 2015, 2016a,b,c, 2018a,b), Chinese from Taiwan region (Arifin and Chien, 2008; Hsu et al., 2010; Wu et al., 2013; Chen et al. 2015, 2016), Brasilian (De Figueirêdo et al., 2011, 2015a,b; Brito, 2015; Brito et al., 2016), Colombian (Gil et al., 2009, 2012, 2014), Italian (Errico and Rong, 2012; Errico et al., 2013, 2015) and Mexican (GutiérrezGuerra et al., 2009; Torres-Ortega et al., 2014; Segovia-Hernández et al., 2014) teams and recently many teams from mainland China (Lei et al., 2002, 2014; Li et al., 2016, 2017; Han et al. 2015; Luo et al., 2014, 2015; Wang et al., 2012, 2015a, 2016, 2018a,b; Zhang et al., 2014, 2017, 2018a,b; You et al. 2017a,b, 2018; Huang et al., 2016; Bai et al., 2017; Zhao et al., 2017a,b, 2018; Gu et al., 2018a). Together with other teams, they consolidate the active community of extractive distillation. Other teams have specialized in separations with ionic liquids, out of our scope (see the review articles by Pereiro et al. (2012) and Lei et al. (2014), with low transition temperature mixtures/deep eutectic solvents (Rodríguez et al., 2015; Rodriguez and Kroon, 2015; Peng et al., 2017; Bai et al., 2017; Zhang et al., 2017; Dongmin and Yanhong, 2018) or intensified with reaction (Espinosa, 2002; Chin et al., 2006).

Extractive distillation is mentioned in all books relative to distillation (Stichlmair and Fair, 1998; Doherty and Malone, 2001; Petlyuk, 2004; Gerbaud and Rodriguez-donis, 2014). It is given a significant place in Lang's book chapter (Lang, 1992), Mujtaba's book (2004), Diwekar's 2nd edition book (2012) and in Luyben and Chien's monograph (2010) where they analyse feasibility, process operation, optimization and control of the separation of several minimum or maximum boiling azeotropes with heavy or light entrainers (1.0-1a class diagrams). Some issues are not discussed in this article, as they have been surveyed in Lei et al. (2003), like tray configurations, processes using solid salts with entrainer or ionic liquid entrainers (Pereiro et al., 2012; Lei et al., 2014), reactive extractive distillation, entrainer selectivity, experimental measurement techniques and computer aided molecular design of extractive entrainers (Pretel et al., 1994; Wu et al., 2007; Kossack et al., 2008). Some new topics, like the use of low transition temperature mixtures/deep eutectic solvents as entrainers (Rodríguez et al., 2015; Rodriguez and Kroon, 2015; Peng et al., 2017; Bai et al., 2017; Zhang et al., 2017; Dongmin and Yanhong, 2018) can be handled with the material in this review, as these entrainers behave like other heavy entrainers.

Other configurations have been proposed in order to reduce the energy consumption and increase the thermodynamic efficiency of the extractive distillation, like batch extractive middle vessel column (Safrit and Westerberg, 1997a,b; Safrit et al. 1995; Hilmen et al., 1997; Warter and Stichlmair, 1999; Low and Sorensen, 2002; Cui et al., 2002), column with partial condenser (Taylor and Wankat, 2004), thermal integration secondary reflux and vaporization (Batista and Meirelles, 1997), thermally coupled extractive and regeneration columns (Errico and Rong, 2012; You et al., 2016b), or double boiler batch column configurations (Hua et al., 2007), Petlyuk column with complex arrangements (Timoshenko et al., 2005; Gutiérrez-Guerra et al., 2009), or extractive dividing-wall column configuration (Midori et al., 2000; Bravo-Bravo et al., 2010; Yildirim et al., 2011; Kiss and Ignat, 2012; Kiss and Suszwalak, 2012; Xia et al., 2012; Wu et al., 2013; Modla, 2013; Sun et al., 2014; Zhang et al., 2014; Luo et al., 2015; Huang et al., 2016; Patrascu et al., 2017, and more).

The industrial scale production of extractive distillation columns can reach hundreds of kilotons per year in $2.5 \mathrm{~m}$ diameter columns (Lei et al., 2003). Several studies hint that extractive distillation is more profitable than heterogeneous azeotropic distillation for many systems, in particular for the dehydration of aliphatic alcohols. As an example, the separation of the 2-propanol-water mixture with DMSO gives a $32.7 \%$ gain in total annual cost (TAC) (Arifin and Chien, 2008). Due to the growing interest in biofuel production, the dehydration of ethanol, of isopropyl alcohol (syn. 2-propanol); of other alcohols and ethers, and the recovery of isopropyl ether, of acetone from various binary mixtures have been studied. Tables in Supplementary materials collect more than fifty mixtures with nearly 250 references, ordered by the extractive separation classes described later in this review.

\section{Extractive process feasibility assessment}

Owing to the importance of the entrainer E selection upon the performance of the extractive distillation process for the separation of a binary mixture A-B (Benedict and Rubbin, 1945; Momoh, 1991), we now discuss it in the context of building a classification of suitable extractive distillation classes (Section 2.6). Each class describes a ternary diagram A-B-E that match the general feasibility criterion (Section 2.5), hinting at the attainable products and relevant process configuration. Heavy, intermediate, light boiling or heterogeneous entrain- 
ers are all found to be suitable under specific conditions for performing extractive distillation. Topics about the process synthesis, design, optimization and control are discussed in Section 3.

We first consider thermodynamics related properties, but we leave out of our discussions several important features such as being available on the market, inexpensive, stable, non-toxic, non-flammable or non-corrosive. Then, the most awaited feature of the entrainer $E$ is its selectivity (Section 2.1), through preferential interaction with either A or B. Recycling of $\mathrm{E}$ is most recommended with the help of the regeneration column in continuous or regeneration step in batch. It will be eased if $\mathrm{E}$ has a high relative volatility with the nonpreferentially interacting compound B or A. Other properties may influence the process efficiency: a low molar volume is sought especially in batch extractive distillation as the entrainer accumulates in the boiler; whereas low heat capacity and vaporization enthalpy will attenuate the energy demand increase. Often, but not exclusively (Rodríguez-Donis and Gerbaud, 2010), the entrainer should not form any new azeotrope with $A$ or $B$, which is usually the case when its boiling temperature is more than $30-50^{\circ} \mathrm{C}$ different from that of $\mathrm{A}$ or $\mathrm{B}$ (Seider et al., 1997). This criterion favoured strongly the choice of a heavy entrainer, and explained why this type of entrainer was so much studied.

Considering process operating parameters, the most important ones are the entrainer flow rate, often taken as the entrainer-feed flow rate ratio, and the reflux ratio for a rectifier configuration or the reboil ratio for a stripper configuration. Those are discussed in Sections 2.2-2.4 before enouncing the general feasibility criterion (Section 2.5 ) that allows identifying suitable extractive separation classes.

Below we consider only pure compounds as entrainers, but several works have studied the use of mixed entrainers, with approaches similar as those for pure entrainers (Benyahia et al., 2014; Dai et al., 2014; Gil et al., 2014; Sazonova et al., 2016; Zhao et al., 2017b, 2018; Zhang et al., 2018b).

\subsection{Entrainer selectivity and capacity}

The entrainer $E$ should either enhance or reduce the relative volatility $\alpha_{A B}$. Assuming that the pure liquid fugacity in a reference state is equal to the vapour pressure $P_{i}^{0}, \alpha_{A B}$ is calculated from the following equation:

$\alpha_{A, B}=\frac{K_{A}}{K_{B}}=\frac{y_{A} / x_{A}}{y_{B} / x_{B}}=\frac{\gamma_{A} \cdot P_{A}^{o}}{\gamma_{B} \cdot P_{B}^{o}}$

where $K_{i}, x_{i}, y_{i}$ and $\gamma_{i}$ are the component I equilibrium constant, liquid molar fraction, vapour molar fraction and activity coefficient respectively.

For small temperature changes, the ratio $P_{A}^{0} / P_{B}^{0}$ remains almost constant and the activity coefficients ratio $\gamma_{A} / \gamma_{B}$ dominates the changes in $\alpha_{A B}$. In the presence of entrainer, we can define the selectivity $S_{A, B}$ :

$S_{A, B}=\frac{\gamma_{A, E}}{\gamma_{B, E}}$

which under infinite dilution becomes:

$S_{A, B}^{\infty}=\frac{\gamma_{A}^{\infty}}{\gamma^{\infty}}$

$S_{A, B}^{\infty}$ should depart as much as possible from unity (Momoh, 1991) and can be used to compare entrainers leading to the same process flowsheet configuration (Kossack et al., 2008). Alternatively, the relative volatility value can be used, either computed under infinite dilution $\alpha_{A B}^{\infty}$ (Gmehling and Möllmann, 1998) or for different entrainer concentrations or as an average value over some entrainer composition ranges like $\mathrm{x}_{\mathrm{E}} \cong 0, \mathrm{x}_{\mathrm{E}}=0.5$ and $\mathrm{x}_{\mathrm{E}} \cong 1$ entrainer content (RodríguezDonis et al., 2012b). The recommendation of Kossack et al. (2008) about the process structure comes from the observation that some entrainers enhance the volatility, while others reduce it; leading to different products and process configurations for the same mixture (Laroche et al., 1992a; Gmehling and Möllmann, 1998; Van Kaam et al., 2008; Kossack et al., 2008; Luyben and Chien, 2010). The choice of the process configuration and direct or indirect separation type will be evidenced straightforwardly when discussing volatility order regions, the general feasibility criterion and the extractive separation classes.

Belonging to the most studied (1.0-1a) extractive separation class, the separation of the minimum boiling azeotrope acetone (A)-methanol (B) enables recovering either A or B depending on the entrainer and whether they enhance or reduce $\alpha_{\mathrm{A}}$. Kossack et al. (2008) studied 14 such entrainers they recommended to rank the entrainers not on the basis of the selectivity alone but through the product of the entrainer selectivity with the entrainer capacity. That property called by Pretel et al. (1994) the Solvent Power (be careful that they designate $B$ as more volatile than A in their paper) can be evaluated from the following equation, written here for the less volatile component B:

$C_{B, \text { entrainer }}^{\infty}=\frac{1}{\gamma^{\infty}}$

The smaller the value of the activity coefficient $\gamma_{B}^{\infty}$, the stronger are the interactions between component $\mathrm{B}$ and the entrainer, which results in a larger capacity. Jork et al. (2005) noted that highly selective entrainers often possess a low capacity. $S_{A, B}^{\infty}$ alone does not correlate well with the process total cost (Momoh, 1991). Kossack et al. (2008) found a reasonable agreement between their ranking combining selectivity and capacity and the TAC of optimized process flowsheets. Eqs. (4) and (5) are sometimes corrected with the ratio of the molecular weight of A and B, or B and E respectively (Pretel et al., 1994; Kossack et al., 2008).

\subsection{Minimal entrainer amount}

For the separation of a minimum boiling azeotropic mixture A-B, like ethanol-water, with a heavy entrainer like ethylene glycol (1.0-1a extractive separation class), a minimal entrainer amount exists that is said to 'break' the azeotrope. It is readily computed as the amount above which the azeotrope or univolatility condition $\alpha_{A B}=1$ no longer exists when computed on an entrainer-free basis (Lee and Pahl, 1985). Fig. 2 displays such an example where the UNIFAC Dortmund modified 1993 thermodynamic model (Gmehling et al., 1993) was used to compute the vapour liquid equilibrium.

The addition of $11 \mathrm{~mol} \%$ ethylene glycol makes the minimum boiling azeotrope disappear on an entrainer-free basis on the ethanol-water vapour-liquid equilibrium curve. The entrainer-free molar fractions $x^{+}$and $y^{+}$are molar fractions calculated without the entrainer. For example, in the A-B-E liquid mixture $\{0.3,0.59,0.11\}, x^{+} A=0.3 /(0.3+0.59)=0.337$. The reader is warned that the choice of the thermodynamic model 


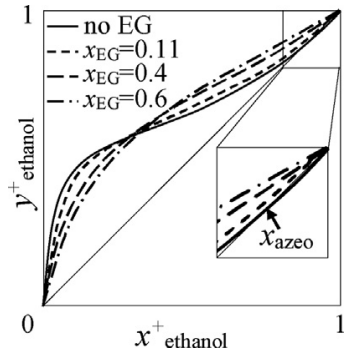

Fig. 2 - Entrainer-free basis ethanol-water equilibrium curve with ethylene glycol (EG) (adapted with permission from Gerbaud and Rodriguez-Donis (2014). Copyright 2014 Elsevier).

may influence the quantitative results in the above equations and should always be validated with experimental data.

\subsection{Isovolatility curves $\alpha_{A B}=$ const}

Isovolatility curves $\alpha_{A B}=$ const are very useful complements to selectivity to rank entrainers as illustrated for the $\operatorname{minT}$ azeotrope ethanol-water separation with either ethylene glycol (EG) or glycerol (Fig. 3). The higher the isovolatility value near the pure entrainer vertex, the better the enhancement of the relative volatility between A and B and likely the easier the separation.

Curves in Fig. 3 were calculated with the UNIFAC Dortmund modified 1993 thermodynamic model. In Fig. 3a, the intersection point $x_{P}$ of the isovolatility curve $\alpha_{A B}=1$ with the triangle edge is $x_{P}=0.11$ for EG. It corresponds to the minimum entrainer amount in Fig. 2 to get rid of the azeotrope on an entrainer free basis. Other comparative examples are found in the literature, like the minT azeotrope 2-propanol-water separation for which several entrainers like DMSO or EG have been proposed for extractive distillation (Gmehling and Möllmann, 1998; Arifin and Chien, 2008; Luyben and Chien, 2010).

Using the results in Fig. 3, the entrainer concentration in $x_{P}$ is lower in the case of EG. Besides, near the pure entrainer vertex, selectivity and relative volatility is higher for EG $\left(S_{A, B}^{\infty}=1.62, \alpha_{A, B}^{\infty} \cong 2.85\right)$ than for glycerol $\left(S_{A, B}^{\infty}=0.81\right.$, $\left.\alpha_{A, B}^{\infty} \cong 1.17\right)$. These figures hint at a possibly easier separation in the extractive distillation column with EG. However, notice that a high selectivity and a smaller entrainer concentration in $x_{P}$ are not always the rule: Luyben and Chien (2010) showed that for the separation of methanol-dimethyl carbonate with aniline or ethylene glycol as heavy entrainers, although ethylene glycol gave the most favorable position of $x_{P \text {,methanol }}$ but it had the least increase of the isovolatility curves near pure entrainer, which resulted in a poor selectivity at infinite dilution.

Using the definition given by Eq. (2), computing univolatility curves $\alpha_{A B}=1$ is routinely done in several simulator packages. Computing isovolatility curves $\alpha_{A B}=$ cst like shown in Fig. 3, is also important but less frequent. When an azeotrope exists, the univolatility curve is evident and can be found. However, as recalled in Reshetov's statistics (Reshetov and Kravchenko, 2007), some zeotropic mixtures may also display univolatility curves that will impact the feasibility (see Table 3 in Section 2.6). Based on our experience, simulator tools can fail to compute all uni- and isovolatility curves, especially those not connected to azeotropes. Hence, recently Shcherbakova et al. (2017) established a novel method for detecting and computing univolatility curves in ternary mixtures. The algorithm is built upon the definition of generalized univolatility surfaces and detects all univolatility curves. These authors illustrated with peculiar ternary diagrams with binary bi-azeotropes and showed that multiple ternary azeotropes, tangential and saddle-node azeotropes could also exist.

Other ternary diagrams, displaying isolines of excess Gibbs free energy and isoselectivity lines, have been used to compare and select entrainers as well (Raeva et al., 2013; Sazonova et al., 2016). These authors used them to study synergetic effects in two-component entrainer mixtures (Raeva et al., 2013). According to them, an entrainer is efficient enough when the maximum difference $\Delta\left(\Delta g^{\text {Excess }}\right)$ between A-E and B-E is approximately equal to or higher than $1000 \mathrm{~J} / \mathrm{mol}$. Considering the same minT azeotrope ethanol-water separation with ethylene glycol as shown in Fig. 3 but with another thermodynamic model and set of parameters, Fig. 4a shows that the threshold of $1000 \mathrm{~J} / \mathrm{mol}$ is almost reached (read horizontally on an iso EG content basis: $+855-(\approx-74)=\approx+929 \mathrm{~J} / \mathrm{mol})$. Fig. $4 \mathrm{~b}$ shows also the isoselectivity $\mathrm{S}=$ cstant diagram. In the composition's areas where $S>1$, separating agent consumptions and relative volatility change in the same way, whilst for fields with $S<1$ an increase in entrainer consumptions leads to a decrease of selectivity, although relative volatility values can stay quite high (Raeva and Sazonova, 2015).
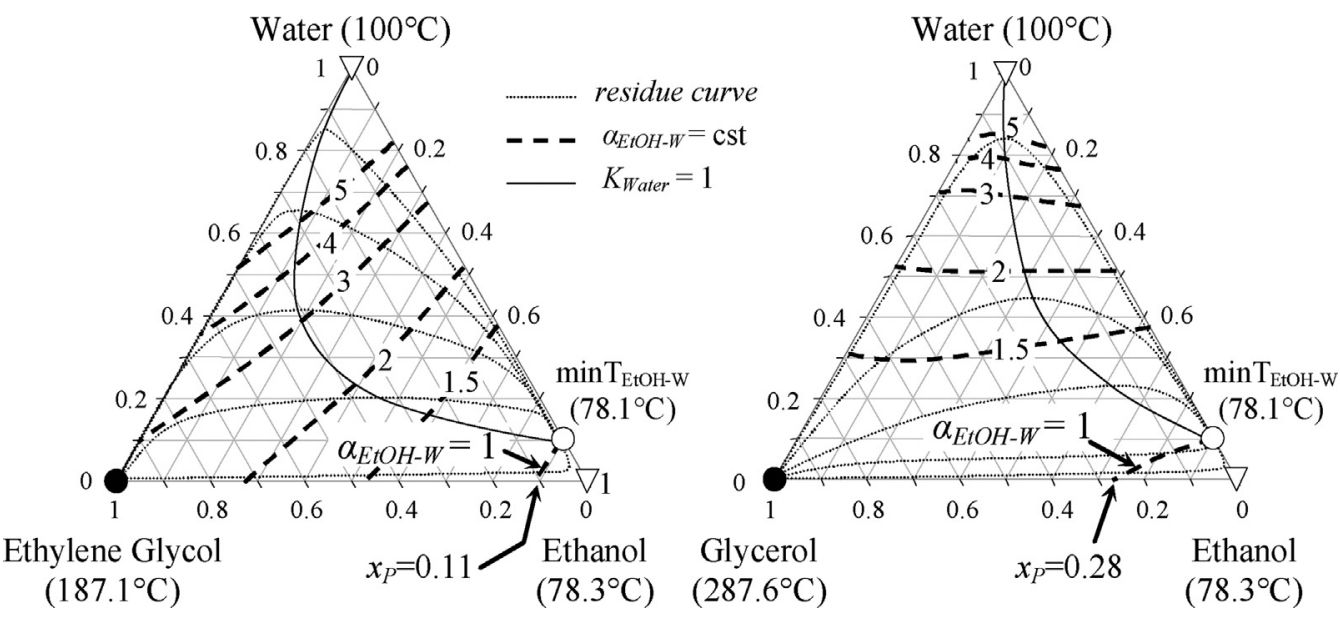

Fig. 3 - (1.0-1a) Residue curve map, isovolatility lines and water unidistribution curve for the ethanol (A)-water (B) with heavy entrainer $(E)$ ethylene glycol or glycerol. (adapted with permission from Gerbaud and Rodriguez-Donis (2014). Copyright 2014 Elsevier). 
(a)

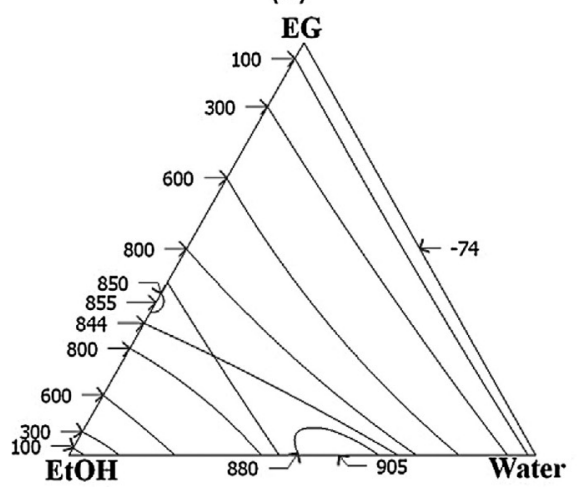

(b)

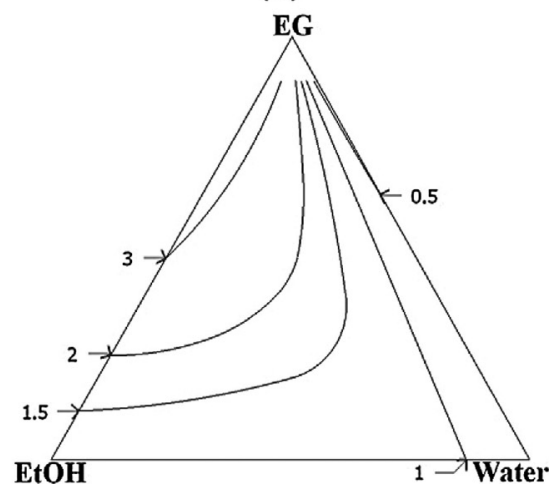

Fig. 4 - Diagrams of excess Gibbs energy isolines $(\mathrm{J} / \mathrm{mol})$ (a) and isoselectivity plots (b) at $101.3 \mathrm{kPa}$ for ethanol-water-ethylene glycol mixture. (Raeva and Sazonova, 2015. Copyright 2015 Waset).

Therefore, one should select the entrainer with the highest selectivity (Sazonova et al. 2014; Sazonova and Raeva, 2015). These results suggest that entrainer selection criteria based on thermodynamic issues as intersection of the isovolatility curve at $\mathrm{x}_{\mathrm{P}}$ and the evaluation of the selectivity at infinite dilution in the entrainer are not conclusive and exclusive of other approaches because the high diversity of the topology of isovolatility curves map (see Fig. 3).

Furthermore, ternary diagrams can give more information about the process. First, they can hint at the easiness of the entrainer regeneration step. When using a heavy entrainer as in Fig. 3, entrainer regeneration is fairly easy as EG and glycerol entrainers have a much higher boiling point than water. This is consistent with the suggestion that a boiling temperature difference between the entrainer and the non-distillate product compound should be higher than $40^{\circ} \mathrm{C}$ (Gmehling and Möllmann, 1998). Second, for intermediate entrainers which inherently have boiling points close to both $\mathrm{A}$ and $\mathrm{B}$, the location of the unidistribution line $K_{E}=1$ should be considered and it should not be too close to the triangle side (Rodríguez-Donis et al., 2012b). As unidistribution curves $K_{I}=1$ describe the locus of residue curves extrema in compound $i$ molar fraction (Kiva et al., 2003), this situation implies residue curves with sharp turns, and more trays and energy would likely be needed to achieve the separation. This behaviour was shown by Rodríguez-Donis et al. (2012b) who compared four entrainers for the batch extractive separation of the maxT azeotrope chloroform-ethyl acetate. They observed that bromochloromethane achieved the highest recovery of chloroform. It was correlated with a small $x_{P}$ intersection value but also with a larger feasible region because of the position of the univolatility line $\alpha_{\mathrm{AE}}=1$ and a more favourable location of unidistribution line $K_{E}=1$ than other entrainers.

\subsection{Univolatility curves $\alpha_{A B}=1$ and achievable product}

The feasibility assessment of the extractive distillation process requires the knowledge of the $\mathrm{A}$ and $\mathrm{B}$ volatility order that is governed by the univolatility curve $\alpha_{A B}=1$. Defined by Eq. (2), univolatility curves $\alpha_{A B}, \alpha_{A E}$ and $\alpha_{B E}$ split the ternary diagrams into volatility order regions (Serafimov, 1970; Laroche et al., 1991; Kiva et al., 2003; Serafimov et al., 2008) and can be used to sketch the topology of residue curve maps and the shape and curvature of residue curves (Wahnschafft and Westerberg, 1993; Kiva et al., 2003). An azeotropic composition belongs to a univolatility curve. But univolatility curves which are not con- nected to azeotropic points may also exist and they occur in both azeotropic and zeotropic ternary diagrams (Kiva et al., 2003; Reshetov and Kravchenko, 2007).

For the most popular extractive separation in industry, namely that of a minT azeotropic mixture A-B with E heavy (ternary Diagram 1.0-1a, Fig. 5a), Laroche et al. (1991) explained that depending on the $\alpha_{\mathrm{AB}}=1$ curve location on the ternary diagram, A was the product when $\alpha_{A B}=1$ intersected the A-E edge and $\mathrm{B}$ was the product when $\alpha_{A B}=1$ intersected the $\mathrm{B}-\mathrm{E}$ edge. Volatility order regions (e.g. $A B E=A$ more volatile than $B$ than $E$ ) are displayed in each diagram with the possible achievable product being underlined, like product ' $A$ ' in $A B E$ region. Later, several authors showed that the achievable product and the minimum entrainer amount could be inferred from the co-analysis of the residue curve map and univolatility curve location for the continuous extractive distillation process (Wahnschafft and Westerberg, 1993; Knapp and Doherty, 1994; Gmehling and Möllmann, 1998; Brüggemann and Marquardt, 2004; Petlyuk et al., 1999, 2015) and the batch extractive distillation process (Lelkes et al., 1998a,c; Frits et al., 2006; Rodríguez-Donis et al., 2009a; Zhan et al., 2018).

Additional information is brought by sketching on ternary diagrams the composition profile in the extractive section (Lelkes et al., 1998a,c; Frits et al., 2006; Rodríguez-Donis et al., 2009a; Petlyuk et al., 2015). They are computed with the help of equations derived later in Section 3.3.3. For example, Fig. 5 displays the influence of the reflux $\mathrm{R}$ and entrainer-feed flow rate $F_{E} / V$ ratios on the extractive section composition profile map for the class 1.0-1a diagrams. The $\mathrm{F}_{\mathrm{E}} / \mathrm{V}$ ratio used here refers to the batch mode of the extractive distillation process.

The residue curve map displays an unstable node minimum boiling azeotropic mixture (Fig. 5a). The heavy boiling entrainer is a stable node. A and B are saddle points, which incidentally prevent their recovery by azeotropic distillation. Considering a direct split in a batch extractive rectifier, one sketches the extractive profile map in Fig. 5b. For an infinitesimal entrainer-feed flow rate ratio $F_{E} / V$ value (Fig. $5 b$ ), the stationary points of the extractive composition profile map coincide with the residue curve stationary points (Fig. 5a). The univolatility curve $\alpha_{A B}=1$ intersects the A-E edge and characterizes a change in volatility order from one side to the other. Volatility order regions (e.g. $A B E=A$ more volatile than $B$ than $E$ ) are displayed, $A B E$ on the left, $B A E$ on the right. As $F_{E} / V$ increases, stationary points $S_{\text {extr }}, S_{B, e x t}$ and $S_{A \text {,ext }}$ move toward $E$. Below the minimal entrainer amount $\left(F_{E} / V\right)_{\min }$ (Fig. $5 \mathrm{c}$ ), the terminal point of the extractive section profiles $\mathrm{SN}_{\text {extr }}$ stays on the univolatility curve. Above $\left(F_{E} / V\right)_{\min }$ (Fig. $\left.5 \mathrm{~d}\right)$, 


\section{0-1a Residue curve map}
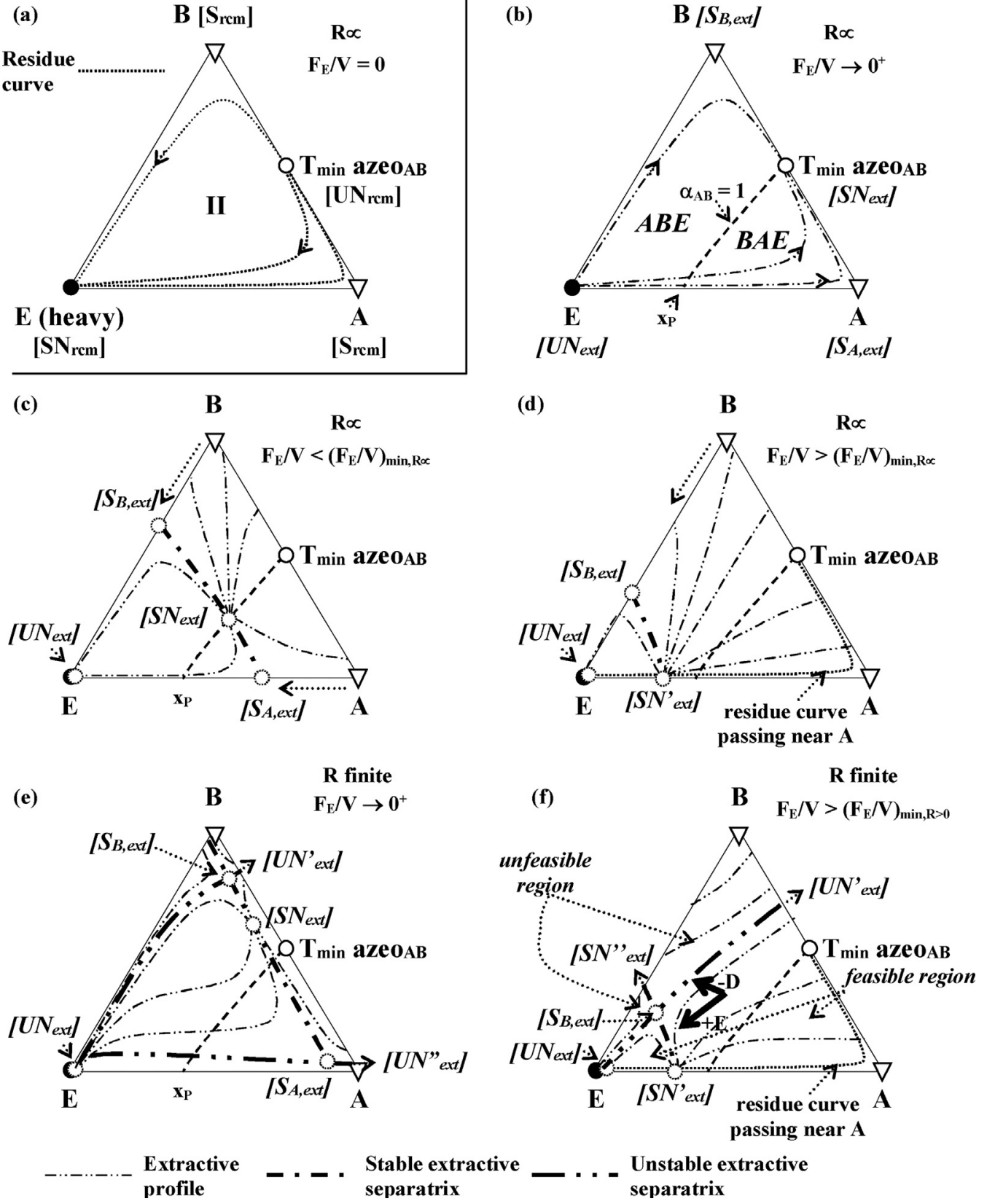

Fig. 5 - Influence of reflux and entrainer-feed flow rate ratios on the extractive section composition profile map for the class 1.0-1a diagrams (adapted with permission from Rodríguez-Donis et al. (2009a). Copyright 2009 American Chemical Society).

$\mathrm{SN}_{\text {extr }}$ leaves the univolatility curve to lie near the $\left[\mathrm{x}_{\mathrm{P}} ; \mathrm{E}\right] \mathrm{seg}-$ ment (Knapp and Doherty, 1994; Frits et al., 2006; Petlyuk et al., 2015). Then all extractive profiles can cross a rectifying profile which reaches the vicinity of the product, A. Under finite reflux ratio, as stationary points move inside and outside the triangle, extractive separatrices appear (Fig. 5e). Above a minimum value $\left(F_{E} / V\right)_{\min , R}>0$, A can be obtained as distillate but the unstable extractive separatrix reduces the feasible region (Fig. 5f). This prevents total recovery in the batch operation mode (Lelkes et al., 1998a; Frits et al., 2006; Rodríguez-Donis et al., 2009a). To conclude, Fig. 5b contains already most of the necessary information: the possible achievable product for class 1.0-1a with $\alpha_{A B}=1$ intersecting the A-E edge is A since the extractive profile stable node $\mathrm{SN}_{\text {extr }}$ must lie in the volatility order region $\mathrm{ABE}$, where $\mathrm{A}$ is the most volatile component. $\mathrm{SN}_{\text {extr }}$ lying in this region happens when $\left(F_{E} / \mathrm{V}\right)>\left(F_{E} / \mathrm{V}\right)_{\min }$.

\section{Extractive profile maps}

(b)

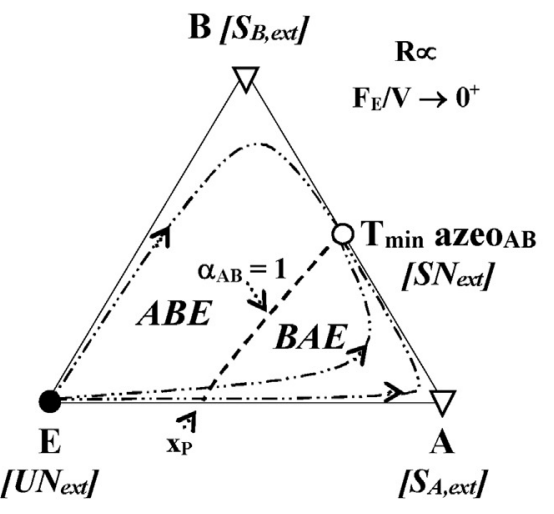

(d)

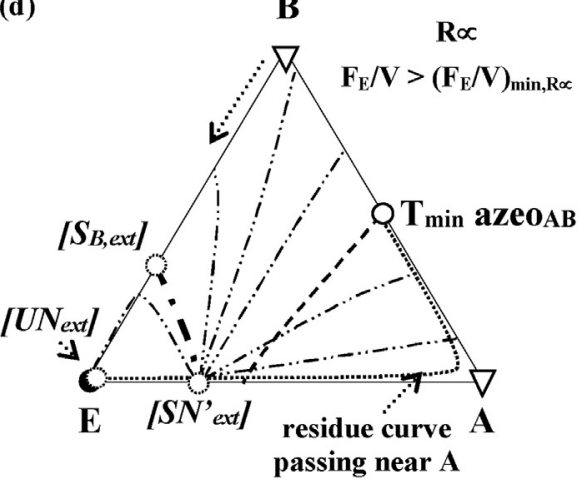
in batch mode by the entrainer-feed flow rate ratio $F_{E} / F$ used in the continuous process, the same analysis holds for the continuous process with a direct split (Knapp and Doherty, 1994; Shen et al., 2013). As the reflux ratio and the entrainer flow rate vary, the locus of the stationary/pinch points describes the so-called pinch branches displayed in Fig. 6 taken from Brüggemann and Marquardt (2004) and Petlyuk et al. (2015) and based on a continuous extractive model.

Petlyuk et al. $(1999,2015)$ used the acetone (1) methanol (2) water (3) 1.0-1a class mixture as illustration and notations $\mathrm{N}_{\mathrm{e}}^{+}$, $\mathrm{N}_{\mathrm{e}}{ }^{-} \mathrm{S}$ e, E/D and L/V in place of ours $\mathrm{SN}_{\text {ext }}, \mathrm{UN}_{\text {ext }}, \mathrm{S}_{\text {ext }}, \mathrm{F}_{\mathrm{E}} / \mathrm{V}$ and $\mathrm{R}$, respectively (Fig. 6a). Brüggemann and Marquardt (2004) displayed another 1.0-1a class mixture turned counter clockwise (Fig. 6b). The stability they refer to is based on the eigenvalues and eigenvectors at the pinch and is the opposite of Petlyuk's 

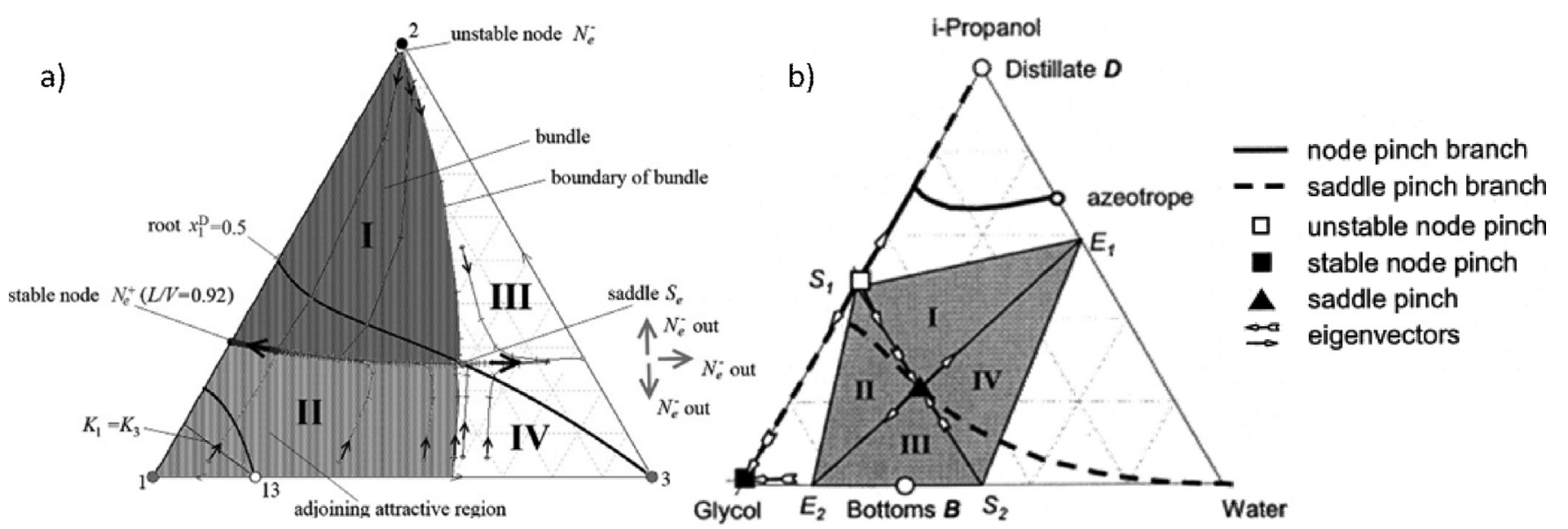

Fig. 6 - Pinch branches and bundle of extractive section trajectories for two class 1.0-1a diagrams. (a) ISS method (Petlyuk et al., 2015, Copyright 2015 Elsevier) (b) RBM method (Brüggemann and Marquardt, 2004, Copyright 2004, Wiley).

work that uses Knapp and Doherty's (1994) reasoning as we did in Fig. 5. Indeed, the stability of the extractive profile map stationary points at the limit $F_{E} \rightarrow 0^{+}$displayed in Fig. $5 b$ is the opposite of the residue curve map stability (Fig. 5a) for a direct split process, since the extractive section profile equations depending on the reflux ratio are computed upward in the column so as to intersect the rectifying section profile (Knapp and Doherty, 1994; Frits et al., 2006; Rodríguez-Donis et al., 2009a). On the other hand, the stability of stationary points remains the same at the limit $\mathrm{F}_{E} \rightarrow \mathrm{O}^{+}$for an indirect split process, where the inverse extractive section profile dependent on the reboil ratio is computed downward in the column so as to intersect the stripping section profile (Rodríguez-Donis et al., 2012a). Knowledge of the stability of stationary points enables the quick estimation of the movement of the extractive profile map stationary points as the entrainer content increases (Fig. $5 \mathrm{c}-\mathrm{f}$ ) and the determination of the location of the extractive stable node enabling a feasible extractive distillation process. Similar figures are found in the literature for the (0.0-1) extractive separation class (low $\alpha$ mixture separation with a heavy entrainer) (Rodríguez-Donis et al., 2009b) and for the (1.0-2) extractive separation class ( $\operatorname{minT}$ azeotrope separation with a light entrainer) (Gerbaud and Rodriguez-donis, 2014; Shen et al., 2015b, 2016).

Following these opening works, a systematic exploration of the feasible separation of minimum or maximum boiling azeotropes with either heavy, intermediate or light entrainers was carried out for the batch extractive distillation process. Rectifier configuration variants (Lang et al., 1994, 1995, 1999, 2000a,b; Lelkes et al., 1998a,b,c, 2002, 2003a,b; Rev et al., 2003) and stripper configuration variants (Varga et al., 2006a,b) were investigated and compared as well as feed location variants (Modla et al., 2003; Kotai and Lang, 2005; Stéger et al., 2005, 2006) and middle vessel column (Safrit et al. 1995; Safrit and Westerberg, 1997a,b; Low and Sorensen, 2002; Cui et al., 2002; Kim et al., 2004; Warter and Stichlmair, 1999, 2004; Kotai and Lang, 2005; Li et al., 2006).

\subsection{General feasibility criterion for extractive distillation with an intermediate feed location}

Based on those works and exploiting results from pioneering works of the 90's in continuous (Laroche et al., 1991, 1992b; Wahnschafft and Westerberg, 1993; Knapp and Doherty, 1994) and in batch (Lelkes et al., 1998a; Frits et al., 2006) that were later confirmed (Kossack et al., 2008; Petlyuk et al., 1999, 2015), a pattern emerged for the rectifier and stripper configuration variants with the entrainer fed at an intermediate location (Fig. 1). A general feasibility criterion was published for the separation by homogeneous extractive distillation of a minT or maxT azeotropic binary mixture A-B with either a heavy (Rodríguez-Donis et al., 2009a), light (Rodríguez-Donis et al., 2012a) or intermediate entrainer E (Rodríguez-Donis et al., $2012 b$ ) or for the separation of low $\alpha$ mixtures (RodríguezDonis et al., 2009b). It states that: "homogeneous extractive distillation of a $A-B$ mixture with entrainer $E$ feeding is feasible if: (1) A or B is the most volatile (respectively the least volatile) component of the mixture $A B E$ in a region where (2) there exists a residue curve connecting $E$ to $A$ or $E$ to $B$ following a decreasing (respectively increasing) temperature direction towards the A or B apex".

The application of this general feasibility criterion tells which component(s) match(es) the criterion and is a possible product; the type of separation and the column configuration and the existence of limiting values for the entrainer flow rate under infinite reflux or reboil ratio operation.

The type of separation is a direct split if the product is the most volatile within the volatility order region, and is withdrawn in the distillate (Fig. 1a and c); or an indirect split if the product is the least volatile and is withdrawn in the column bottom (Fig. 1b and d). Notice that in azeotropic distillation the direct or indirect split terminology refers to the recovery of a product in the distillate or in the bottoms that is the distillation region unstable or stable nodes, respectively. In extractive distillation, the stability information for finding the achievable product is now substituted by the volatility information: for a direct split the distillate product is the most volatile component. For an indirect split the bottom product is the least volatile component. In many extractive distillation processes, like the 1.0-1a separation class (minT separation with heavy $\mathrm{E}$ or maxT with a light $\mathrm{E}$ ), the product is a saddle point of the distillation region; whatever the split. But, for the 1.0-2 extractive distillation case the product is an unstable node (direct split) or a stable node (indirect split). Incidentally, azeotropic distillation is feasible for the 1.0-2 case as well, but in batch mode the feasible region is larger for the extractive distillation process (Lang et al., 1999).

The general criterion was initially enounced for a batch operation process and was also shown to hold for continuous operation as well, but with new limitations: due to an additional section in the continuous process column and 


\begin{tabular}{|c|c|c|c|}
\hline \multirow[b]{3}{*}{ (Heav } & class $(1.0-1 \mathrm{a})-\mathrm{m} 1$ & \multirow{3}{*}{$x_{D, B}=\stackrel{B}{\nabla}$} & class $(1.0-1 \mathrm{a})-\mathrm{m} 2$ \\
\hline & \begin{tabular}{|l|}
$\min T_{A B}+E$ Heavy \\
41 mixtures \\
\end{tabular} & & $\begin{array}{l}\operatorname{minT}_{\mathrm{AB}}+\mathrm{E} \text { Heavy } \\
16 \text { mixtures }\end{array}$ \\
\hline & $\begin{array}{l}\text { Direct split to get } x_{D, A} \\
\text { in } A B E \text { region for } \mathrm{F}_{E} \geq \\
\mathrm{F}_{E} \text { min * }\end{array}$ & & $\begin{array}{l}\text { Direct split to get } x_{D, B} \\
\text { in } B A E \text { region for } \mathrm{F}_{\mathrm{E}} \geq \\
\mathrm{F}_{\mathrm{E}} \mathrm{min} \text { * }\end{array}$ \\
\hline \multirow[b]{3}{*}{$\underbrace{\operatorname{mint}_{\mathrm{AB}}}_{\substack{\left[S N_{\text {extr, },}\right]_{\text {range }} \\
\text { (Light) }}}$} & class (1.0-2)-m1 & \multirow[b]{3}{*}{ } & class $(1.0-2)-\mathrm{m} 2$ \\
\hline & $\begin{array}{l}\min T_{A B}+E \text { Light } \\
5 \text { mixtures }\end{array}$ & & $\begin{array}{l}\min T_{A B}+E \text { Light } \\
4 \text { mixtures }\end{array}$ \\
\hline & $\begin{array}{l}\text { Indirect split to get } \\
X_{W, A} \text { in } E B A \text { region for } \\
\mathrm{F}_{\mathrm{E}} \leq \mathrm{F}_{\mathrm{E}} \mathrm{max} * \\
\text { Indirect split to get } \\
X_{W, B} \text { in } E A \underline{B} \text { region, no } \\
\text { limit for } \mathrm{F}_{\mathrm{E}}\end{array}$ & & $\begin{array}{l}\text { Indirect split to get } \\
X_{W, A} \text { in } E B \underline{A} \text { region, no } \\
\text { limit for } \mathrm{F}_{E} \\
\text { Indirect split to get } \\
X_{W, B} \text { in } E A \underline{B} \text { region, for } \\
F_{E} \leq \mathrm{F}_{E} \max ^{*}\end{array}$ \\
\hline \multirow{3}{*}{ 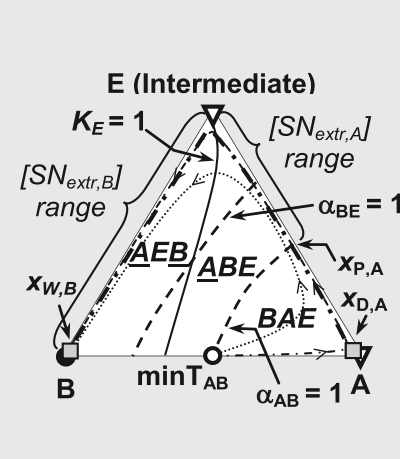 } & class (1.0-1b)-m & \multirow{3}{*}{\multicolumn{2}{|c|}{ 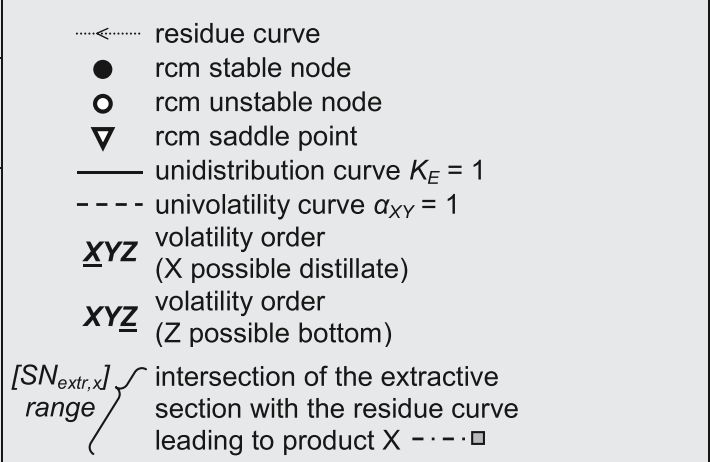 }} \\
\hline & \begin{tabular}{|l|}
$\min T_{A B}+$ \\
Intermediate \\
6 mixtures
\end{tabular} & & \\
\hline & $\begin{array}{l}\text { Direct split to get } x_{D, A} \\
\text { in } A E B \text { and } A B E \\
\text { regions } \\
F_{E} \geq F_{E} \text { min * } \\
\text { Indirect split to get } \\
X_{W, B} \text { in } A E B \text { region, no } \\
\text { limit for } \mathrm{F}_{E}\end{array}$ & & \\
\hline
\end{tabular}

${ }^{*}$ Substitute $\mathrm{F}_{\mathrm{E}}$ by: in batch mode, $\mathrm{F}_{\mathrm{E}} / \mathrm{V}$ (direct split) or $\mathrm{F}_{\mathrm{E}} / \mathrm{L}_{\mathrm{T}}$ (indirect split); in continuous mode, $\mathrm{F}_{\mathrm{E}} / \mathrm{F}_{\mathrm{AB}}$.

the additional requirement that composition profiles in the extractive section intersect with the stripping section composition profile (direct split) or with the rectifying section composition profile (indirect split), the feasible parameter value range in the reflux ratio vs entrainer-feed flow rate ratios diagram is smaller for the continuous process than for the batch process (Shen et al., 2013; Shen and Gerbaud, 2013). Finally, we remark that the feasibility criterion holds for any kind of entrainer, classical organic ones or ionic liquids or deep eutectic solvents.

\subsection{Suitable ternary diagrams for extractive distillation}

It was falsely assumed for decades that the unique feasibility rule for the extractive distillation process was the (1.0-1a) class separation, corresponding to the homogeneous extractive distillation of a minT azeotropic mixture with a heavy entrainer or of a maxT azeotropic mixture with a light entrainer (1.0-1a occurrence $21.6 \%$ among azeotropic diagrams). But some twenty works published between 1990 and 2013 explored which ternary diagrams would be suited for extractive distillation (Laroche et al., 1991, 1992a,b; Wahnschafft and Westerberg, 1993; Knapp and Doherty, 1994; Petlyuk et al.,
1999, 2015; Serafimov et al., 2008; Kotai and Lang, 2005; Kotai et al., 2007; Lang, 1992; Lang et al., 1994, 1995, 1999, 2000a,b; Lelkes et al., 1998a,b,c, 2002, 2003a,b; Modla et al., 2001, 2003; Rev et al., 2003; Stéger et al., 2005, 2006; Varga et al., 2006a,b) until the general feasibility rules discussed in the previous section were enounced (Rodríguez-Donis et al., 2009a,b, 2010, 2012a,b; Gerbaud and Rodriguez-donis, 2014; Shen et al., 2013, 2015b, 2016; Shen and Gerbaud, 2013). They have shown that up to $53 \%$ of azeotropic ternary mixtures are suitable for extractive distillation, with light, intermediate or heavy entrainers, homogeneous or heterogeneous, forming or not new azeotropes.

For homogeneous extractive distillation with an entrainer forming no new azeotrope, the feasible diagrams belong to the following classes: (1.0-1a) (separation of minT with $E$ heavy or maxT with E light, occurrence $21.6 \%$ among azeotropic diagrams), (1.0-2) (separation of maxT with E heavy or $\operatorname{minT}$ with E light, occurrence 8.5\%), (1.0-1b) (minT with E light or maxT with E heavy, occurrence $0.4 \%$ ), and (0.0-1) (low $\alpha$ mixture with all type of $\mathrm{E}$, see occurrences in Reshetov and Kravchenko (2007). Besides, when the entrainer forms a new azeotrope, ternary diagrams classes (2.0-1) (occurrence $0.6 \%),(2.0-2 a)$ (occurrence 0.4\%), (2.0-2b) (occurrence 21.0\%) and (2.0-2c) (occurrence $0.9 \%$ ) are suitable as well (Rodríguez- 
Donis et al., 2010), but only for batch distillation, since the new azeotrope prevents a recycle of high purity entrainer in the regeneration column of the continuous process.

For heterogeneous extractive distillation, the (1.0-1b) (occurrence 0.4\%), (2.0-2b) (occurrence 21.0\%), (2.0-2c) (occurrence $0.9 \%$ ) and $2.1-2 \mathrm{~b}$ (occurrence $4.0 \%$ ) classes have also been found suitable for a feasible direct split extractive distillation process (Modla et al., 2003; Rodríguez-Donis et al., 2003, 2007; Van Kaam et al., 2008), since they can exhibit either an unstable or a saddle heteroazeotrope between $\mathrm{E}$ and either $\mathrm{A}$ or $\mathrm{B}$, that is the most volatile product.

Tables 1-5 below describe what we call extractive separation classes. Based on Serafimov's ternary diagram classification, they summarize the achievable products, limits for the reflux (direct split) or the reboil (indirect split) ratio for each ternary diagram leading to a feasible process. Tables 1-3 refers to the separation of minimum boiling azeotrope $\min _{\mathrm{AB}}$, maximum boiling azeotrope $\operatorname{maxT}_{\mathrm{AB}}$ and low relative volatility mixtures low $\alpha_{\mathrm{AB}}$ respectively. Table 4 displays diagrams suitable for homogeneous batch extractive distillation when the entrainer forms a new azeotrope. Table 5 displays diagrams suitable for heterogeneous extractive distillation.

In addition to the distinction of the minT or maxT separation cases labelled with a small ' $m$ ' or capital ' $M$ ' respectively, we distinguish subcases ' 1 ' and ' 2 ' when there exist two dif- ferent univolatility curve locations, intersecting either the A-E edge or the B-E edge respectively, and leading to different volatility order regions and different products (Kiva et al., 2003). Volatility order regions (e.g. $A B E=A$ more volatile than $B$ than $E$ ) are displayed in each diagram with the possible achievable product being underlined, like product ' $A$ ' in $A B E$ region. If the product is the most (resp. least) volatile compound, it requires a direct (resp. indirect) split extractive column configuration (see Fig. 1). Each diagram also displays sketches of the residue curves with the stationary points (black and white circle for stable and unstable nodes respectively, reversed triangle for saddle point). For each subcase ' 1 ' or ' 2 ', the approximate location of the univolatility curve $\alpha_{A B}=1$ is displayed along with the ranges of the extractive stable node $\mathrm{SN}_{\text {extr,X }}$ to obtain product ' $\mathrm{X}$ '. Possible limiting values for the entrainer-feed flow rate ratio value are deduced from the extractive stable node location range. The approximate location of the residue curve assimilated to the section composition profile reaching the product either in the distillate $x_{D}$ (a rectification section for the direct split) or in the bottom $x_{B}$ (a stripping section for the indirect split) is also given (dotted-stripped bold residue curve).

For illustration of how to use these tables, consider the top left sketch in Table 1 class (1.0-1a)-m1. Like all (1.0-1a) classes, it refers to the separation of a minimum boiling azeotrope $A-B\left(\min _{A B}\right)$ with a heavy entrainer E. 40 mixtures have been

Table 2 - Extractive separation classes for the extractive distillation of maximum boiling azeotrope.

\begin{tabular}{|c|c|c|c|}
\hline \multirow{3}{*}{$\underset{B}{\alpha_{A B}=1}$} & class $(1.0-1 \mathrm{a})-\mathrm{M} 1$ & \multirow{3}{*}{ Pange } & \multirow{2}{*}{$\begin{array}{l}\text { class (1.0-1a)-M2 } \\
\operatorname{maxT}_{A B}+E \text { Light } \\
1 \text { mixture }\end{array}$} \\
\hline & $\begin{array}{l}\text { max } T_{A B}+E \text { Light } \\
2 \text { mixtures } \\
\end{array}$ & & \\
\hline & $\begin{array}{l}\text { Indirect split to get } \\
x_{W, A} \text { in } E B A \text { region for } \\
F_{E} \geq F_{E} \text { min * }\end{array}$ & & $\begin{array}{l}\text { Indirect split to get } \\
x_{W, B} \text { in } E A \underline{B} \text { region for } \\
F_{E} \geq F_{E} \text { min * }\end{array}$ \\
\hline \multirow{3}{*}{ (Heavy) } & class (1.0-2)-M1 & \multirow{3}{*}{ (Heavy) } & class (1.0-2)-M2 \\
\hline & $\begin{array}{l}\text { maxT } T_{A B}+E \text { Heavy } \\
3 \text { mixtures }\end{array}$ & & $\begin{array}{l}\text { max } T_{A B}+E \text { Heavy } \\
2 \text { mixtures }\end{array}$ \\
\hline & $\begin{array}{l}\text { Direct split to get } x_{D, A} \\
\text { in } \underline{A B E} \text { region for } \mathrm{F}_{\mathrm{E}} \leq \\
\mathrm{F}_{\mathrm{E}} \mathrm{max} \text { * } \\
\text { Direct split to get } x_{D, B} \\
\text { in } \underline{B A E \text { region, no }} \\
\text { limit for } \mathrm{F}_{\mathrm{E}}\end{array}$ & & $\begin{array}{l}\text { Direct split to get } x_{D, A} \\
\text { in } \underline{A B E} \text { region, no } \\
\text { limit for } \mathrm{F}_{E} \\
\text { Direct split to get } x_{D, B} \\
\text { in } \underline{B} A E \text { region for } \\
\mathrm{F}_{E} \leq \mathrm{F}_{E} \text { max * }\end{array}$ \\
\hline \multirow{3}{*}{ E (Intermediate) } & class $(1.0-1 b)-M$ & \multirow{3}{*}{\multicolumn{2}{|c|}{ 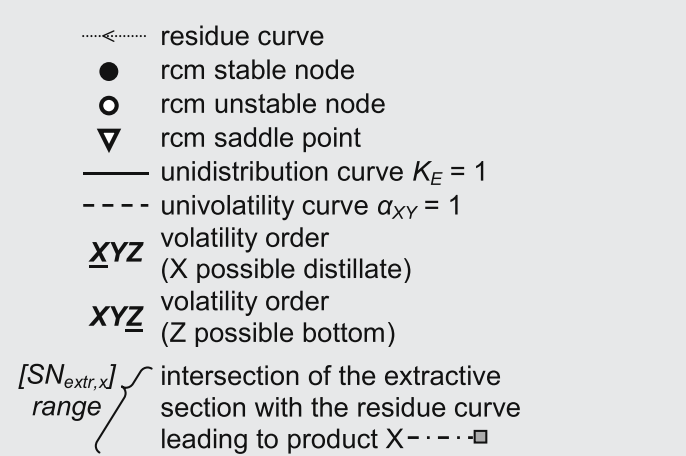 }} \\
\hline & $\begin{array}{l}\max _{\mathrm{AB}}+ \\
\text { Intermediate } \\
1 \text { mixture }\end{array}$ & & \\
\hline & $\begin{array}{l}\text { Direct split to get } \\
X_{D, A} \text { in } \underline{A} E \underline{B} \text { region, } \\
\text { no limit for } \mathrm{F}_{\mathrm{E}} \\
\text { Indirect split to get } \\
X_{W, B} \text { in } \underline{A} E \underline{B} \text { and } \\
E A \underline{B} \text { regions for } \\
\mathrm{F}_{\mathrm{E}} \geq \mathrm{F}_{\mathrm{E}} \mathrm{min}^{*}\end{array}$ & & \\
\hline
\end{tabular}




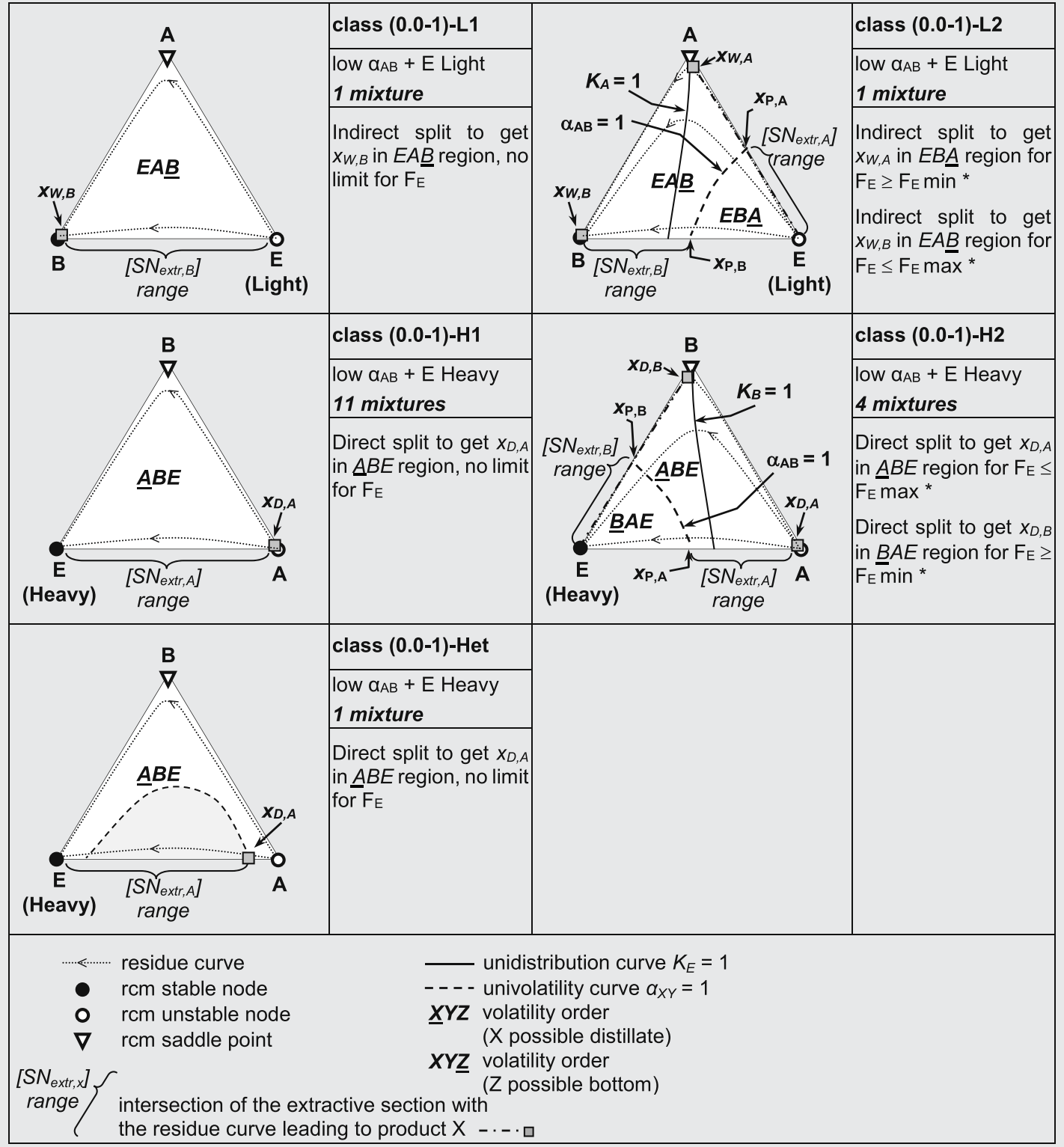

*Substitute $\mathrm{F}_{\mathrm{E}}$ by: in batch mode, $\mathrm{F}_{\mathrm{E}} / \mathrm{V}$ (direct split) or $\mathrm{F}_{\mathrm{E}} / \mathrm{L}_{\mathrm{T}}$ (indirect split); in continuous mode, $\mathrm{F}_{\mathrm{E}} / \mathrm{F}_{\mathrm{AB}}$.

found in the open literature and references are listed in the Supplementary material file. A is the lightest and drawn at the bottom right of the triangle. Going counter clockwise, B is the second in pure component boiling temperature and is at the triangle top and $\mathrm{E}$ is the heaviest boiling and is at the bottom left. The univolatility curve $\alpha_{\mathrm{AB}}=1$ starts at the azeotrope and reaches the A-E edge. As explained in Fig. 5, A is the distillate (a possible location $x_{D, A}$ is drawn) since the extractive profile stable node $\mathrm{SN}_{\text {extr }}$ must lie in the volatility order region $A B E$, where $A$ is the most volatile, in order to intersect a composition profile in the rectifying section (dotted-stripped bold curve) that will reach $x_{D, A}$. The $S_{\text {extr }}$ range is also displayed in accordance with the explanation related to Fig. 5: $\mathrm{SN}_{\text {extr }}$ lies in the $A B E$ region when $F_{E} \geq F_{E \text {,min }}$, so there always exists a minimum entrainer flow rate to achieve the separation. For other classes like class (1.0-2)- $\mathrm{m} 1$, related to $\min _{\mathrm{AB}}$ separation with a light entrainer $\mathrm{E}$, there can be different products, here $\mathrm{A}$ or $\mathrm{B}$, depending on the volatility order regions. Here both products are the less volatile and are thus recovered in the bottoms labelled $\mathrm{x}_{\mathrm{W}, \mathrm{A}}$ or $\mathrm{x}_{\mathrm{W}, \mathrm{B}}$ using and indirect split col- umn configuration (see Fig. 1). Once the $S_{\text {ext,B }}$ lies in the $E A B$ region, no limit exist to recover $\mathrm{x}_{\mathrm{W}, \mathrm{B}}$, even no limiting entrainer flow rate. This is expected since $B$ is the stable node of the residue curve map. In this case with no entrainer fed, we just have an azeotropic distillation column with only a rectifying and a stripping section. The entrainer is fed directly with the main feed $\mathrm{F}_{\mathrm{AB}}$. On the contrary, once the $\mathrm{SN}_{\text {ext, } \mathrm{A}}$ lies in the $\mathrm{EBA}$ region, getting $\mathrm{x}_{\mathrm{W}, \mathrm{A}}$ requires $\mathrm{F}_{\mathrm{E}}$ below a maximal limit $\mathrm{F}_{\mathrm{E}, \max }$. Again, azeotropic distillation can also be used since A is a stable node. However, the product regions are different between the ED and the azeotropic distillation, being governed by two different frontiers; respectively the extractive section separatrix and the distillation boundary (see Shen and Gerbaud, 2013; Shen et al., 2013 for details).

The Supplementary material file provides an extensive list of mixture examples taken from the literature for the extractive distillation cases displayed in Tables 1-5. More than 70 binary mixtures (minimum boiling and maximum boiling azeotropes, low relative volatility mixtures) with light, intermediate or heavy boiling entrainers are listed with nearly 


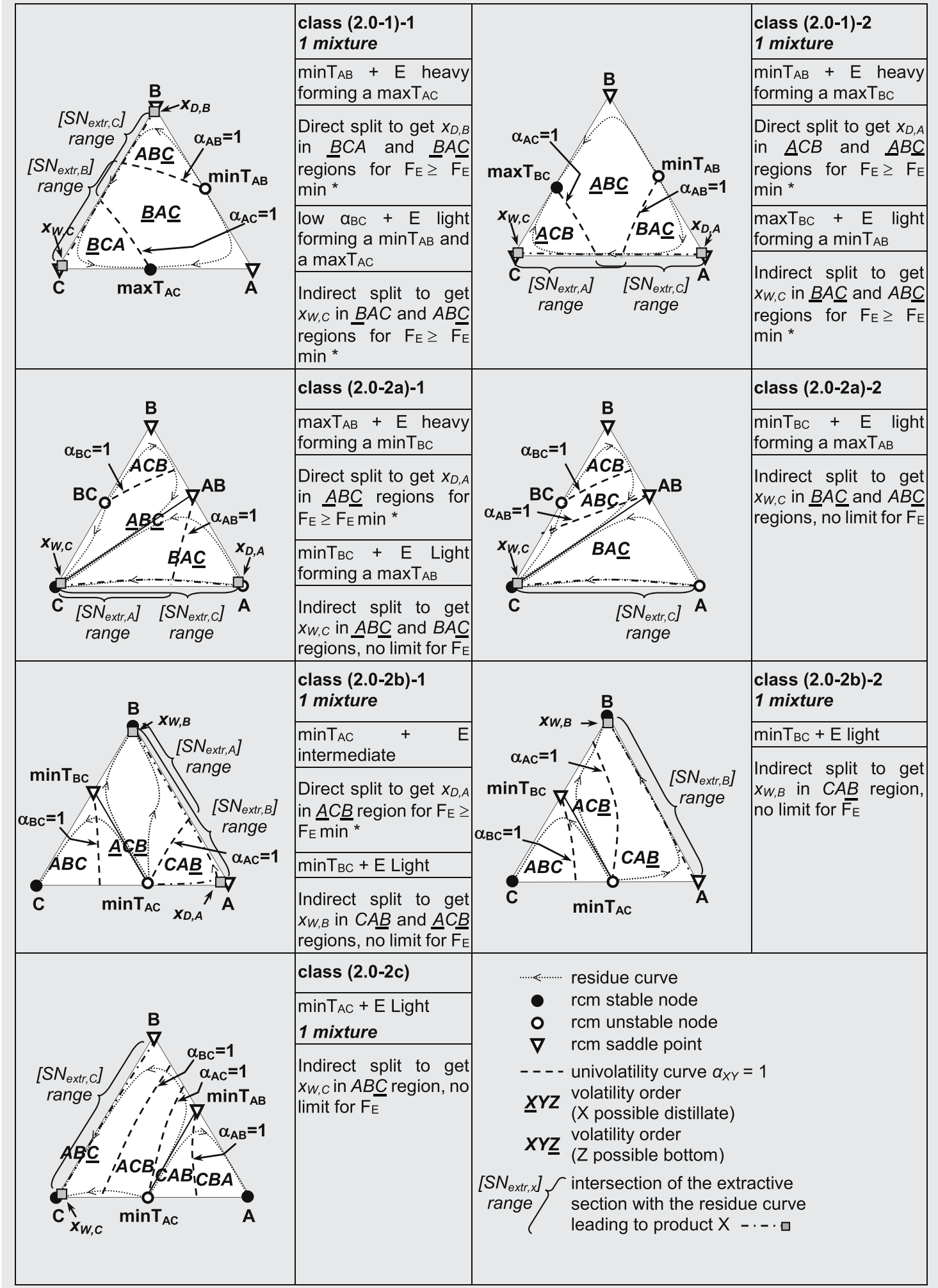

*Substitute $\mathrm{F}_{\mathrm{E}}$ by: in batch mode, $\mathrm{F}_{\mathrm{E}} / \mathrm{V}$ (direct split) or $\mathrm{F}_{\mathrm{E}} / \mathrm{L}_{\mathrm{T}}$ (indirect split); in continuous mode, $\mathrm{F}_{\mathrm{E}} / \mathrm{F}_{\mathrm{AB}}$.

225 references. Tables 1-5 display also the number of binary mixtures relevant for each extractive distillation class and presented in Supplementary material.

For the (1.0-2) and the (1.0-1b) diagrams, other univolatility curves $\alpha_{\mathrm{AE}}=1$ or $\alpha_{\mathrm{BE}}=1$ may exist (see Kiva et al., 2003) but they are not displayed since they do not affect the application of the feasibility criterion.

Here are some additional comments about these tables.
- For the (1.0-1a) diagram, the general behaviour with the reflux ratio and the entrainer-feed flow rate ratio has been given in Figs. 5 and 6. Because the azeotrope is a residue curve map node, the residue curve that enables reaching the product may come near the product vertex but then turn towards the azeotrope. Besides, it is necessary to obtain the extractive stable node as near as possible to the E-product edge, so as to intersect a residue curve reaching near the 
pure product vertex. Otherwise, because the azeotrope is a residue curve map node, the residue curve may approach the product vertex but achieve purity lower than expected. Those aspects are later discussed in the section concerning the operation conditions.

- For the (1.0-2) diagram, the general behaviour with the reflux ratio and the entrainer-feed flow rate ratio similar to Fig. 5, has also been published (Shen, 2012; Gerbaud and Rodriguez-donis, 2014; Shen et al., 2015a). Two other comments are worthy. First, two products are achievable, one with a maximum limit for the entrainer-feed flow rate ratio. However, considering the separation class (1.0-2)-M1 as an example, the maximum limit for separating $\mathrm{A}$ also sets a minimum limit to be sure to obtain B exclusively, especially under finite reflux ratio (Rodríguez-Donis et al., 2009a, 2012a). Second, Laroche et al. (1992b) noticed that conventional azeotropic distillation can also be used to recover one of the unstable nodes A or B. For example, azeotropic batch distillation was feasible for the ternary system maxT acetone (A)-chloroform (B)-benzene (E) (class (1.0-2)-M2), thanks to the curvature of the stable separatrix (Van Dongen and Doherty, 1985b; Bernot et al., 1991). Lang et al. (2000a, 2000b) also showed for this mixture, that batch extractive distillation performed better than azeotropic distillation,

Table 5 - Extractive separation classes for the extractive distillation with heterogeneous entrainers.

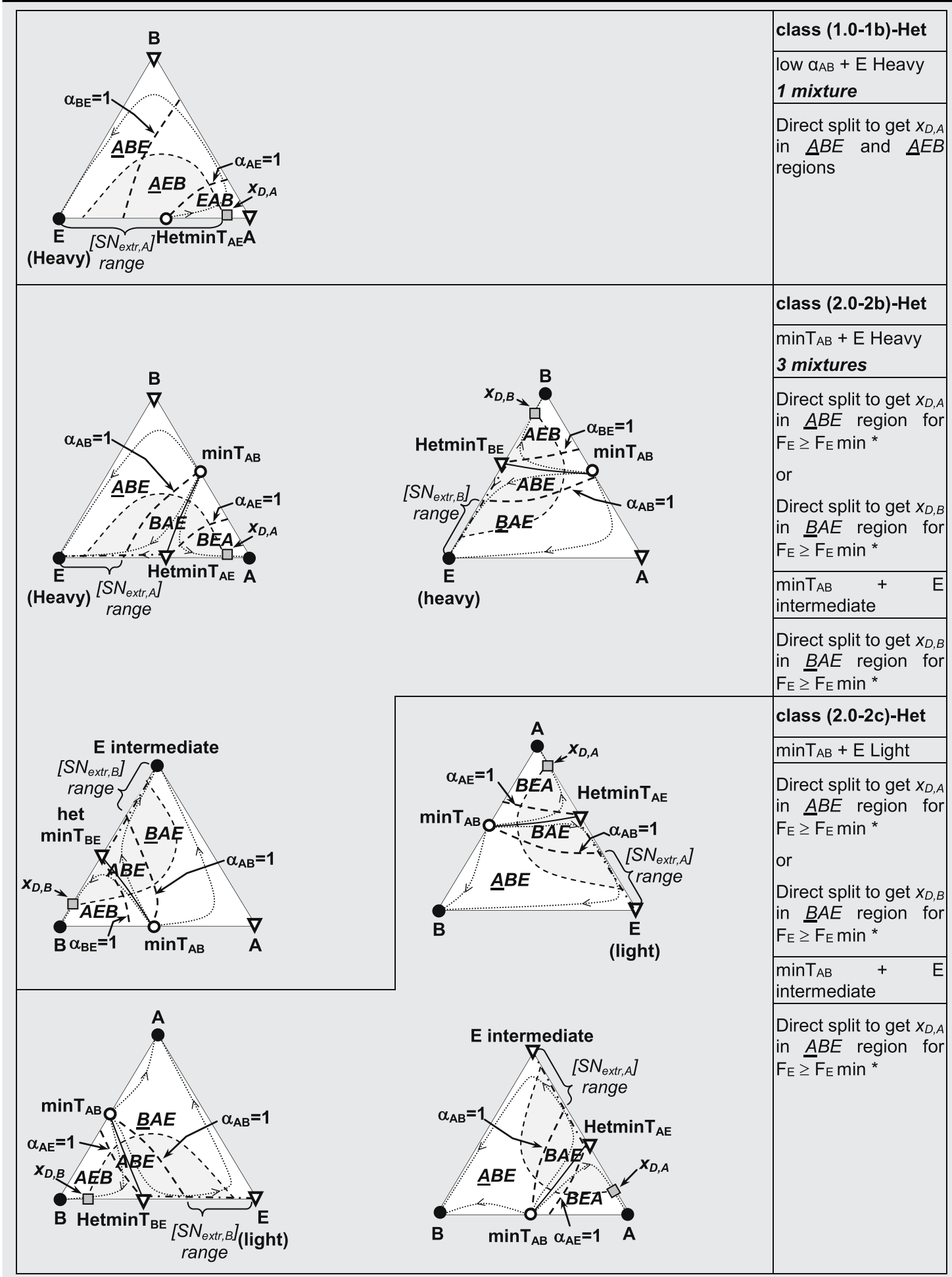




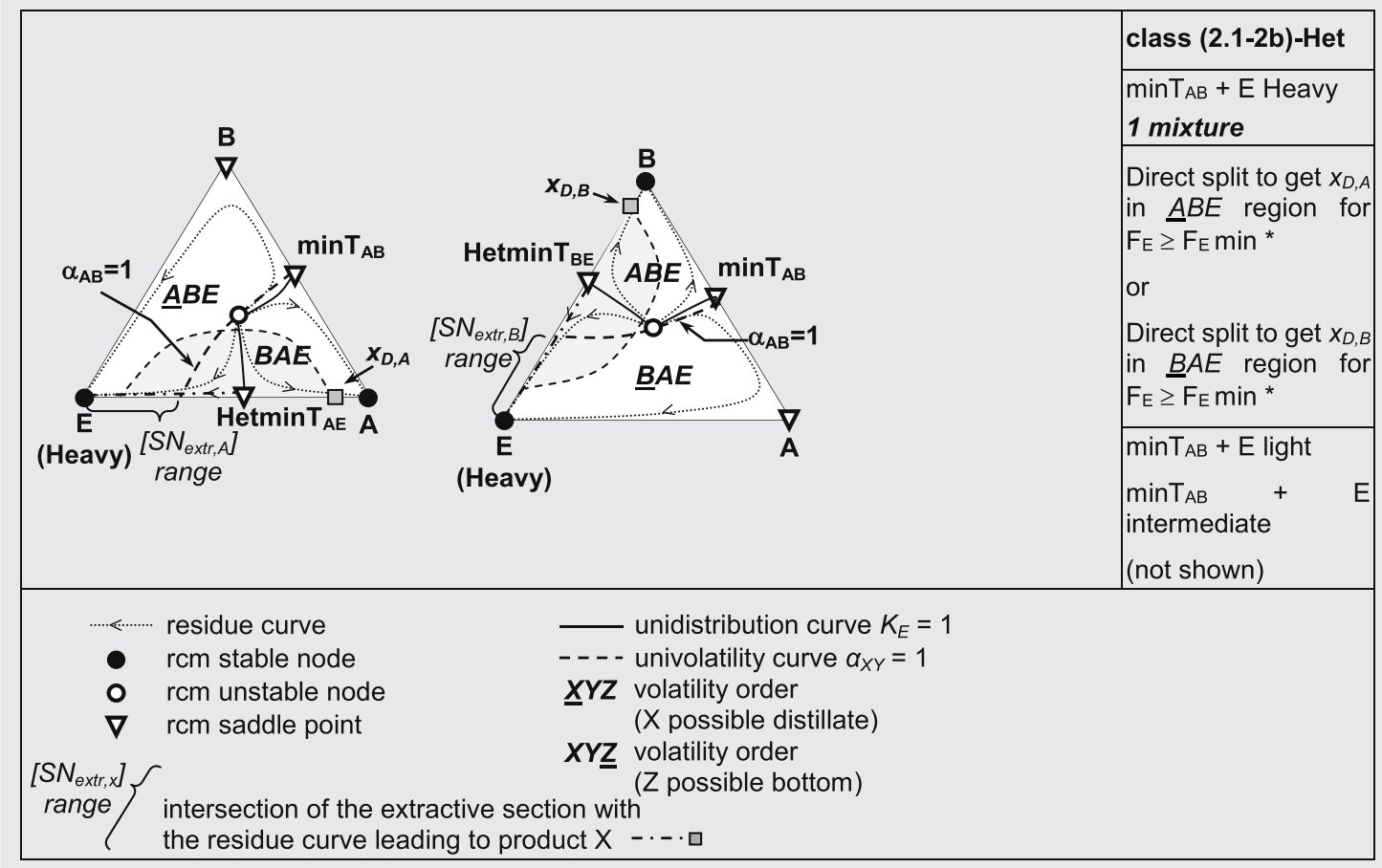

*Substitute $\mathrm{F}_{\mathrm{E}}$ by: in batch mode, $\mathrm{F}_{\mathrm{E}} / \mathrm{V}$ (direct split) or $\mathrm{F}_{\mathrm{E}} / \mathrm{L}_{\mathrm{T}}$ (indirect split); in continuous mode, $\mathrm{F}_{\mathrm{E}} / \mathrm{F}_{\mathrm{AB}}$.

because the feeding of the entrainer generates an extractive separatrix that is also curved and is closer to the B-E side than the rcm stable separatrix, thus increasing the feasible region in which $\mathrm{A}$ is the unstable node. Hence, the extractive distillation alternative improves the recovery yield of component A as the first distillate cut (Lang et al., 2000a, 2000b; Rodríguez-Donis et al., 2009a).

- For the (1.0-1b) diagram, the location of the unidistribution line $K_{E}=1$ and the value of the relative volatility ratio $\alpha_{\mathrm{AE}} / \alpha_{\mathrm{BE}}$ should be looked at when selecting an entrainer, as discussed in Section 2.2, since it hints at the easiness of the entrainer regeneration and helps to decide which component is the easiest to be recovered as product (Rodríguez-Donis et al., 2012b). Laroche et al. (1992b) also noticed that conventional azeotropic distillation can be used to recover the stable node B by using an indirect split.

Note that the number of maximum azeotrope separation case studies investigated in the literature is limited to seven as far as we know, the last one in 2015 (Yu et al., 2015) (see Supplementary material).

- For the (0.0-1) diagram, the general behaviour with the reflux ratio and the entrainer-feed flow rate ratio has been published in Rodríguez-Donis et al. (2009a). Reshetov and Kravchenko (2007) have reported detailed statistics about the maximum number and types of possible univolatility curves in zeotropic diagrams: classes (0.0-1)-L1 and $(0.0-1)-\mathrm{H} 1$ amount for $71.6 \%$ of zeotropic ternary mixtures. Classes (0.0-1)-L2 and (0.0-1)-H2 amount for $11.3 \%$ of zeotropic ternary mixtures and show that even without any azeotrope, the univolatility curve $\alpha_{A B}=1$ may exist and affect the extractive distillation process feasibility, enabling to recover either A or B as a product, whereas without univolatility curve $\alpha_{\mathrm{AB}}=1$ as in classes (0.0-1)-L1 and (0.0-1)-H1, only one product can be recovered. The occurrence of other univolatility curves $\alpha_{\mathrm{AE}}=1$ or $\alpha_{\mathrm{BE}}=1$ concerns about $18 \%$ of zeotropic ternary mixtures and behave like the (0.0-1)-L1 and (0.0-1)-H1 classes. The (0.0-1) Het class has been illustrated with the separation of cyclohexane (A)-cyclohexane (B) with ethylene glycol (Yi et al., 2018). EG forms no azeotrope with either A or B but induces LLV regions with each component. The LLV region with cyclohexane exhibits the lowest boiling point and enables recovering $99 \mathrm{~mol} \%$ cyclohexane after decantation at the top of the heterogeneous extractive column.

Diagrams in Table 4 concern the separation with entrainers forming new azeotropes (Rodríguez-Donis et al., 2010). The extractive process is feasible for batch distillation only, since in continuous distillation, the new azeotrope prevents a recycle of high purity entrainer from the regeneration column. Examples are listed in the Supplementary material file.

- Diagrams 2.0-1: there is only one location for the univolatility lines $\alpha_{\mathrm{AB}}$ and $\alpha_{\mathrm{AC}}$, dividing the ternary composition space in three volatility order regions. In this case, the general feasibility criterion is fulfilled for the saddle components B and C. $B$ can be recovered as a distillate product using a direct split if $S N_{\text {ext, } B}$ lies in the BCA or in the BAC volatility order regions, which requires $F_{E}>F_{E, \text { min, } B}$. $C$ can be recovered in the bottoms using an indirect split if $\mathrm{SN}_{\text {ext, } \mathrm{C}}$ lies in the $\mathrm{BAC}$ or the $A B C$ volatility order regions, which requires $F_{E}>F_{E, \text { min, } C}$. Rodríguez-Donis et al. (2010) presented the separation of minT azeotropic mixture methanol (A)-THF (B) by continuous feeding of heavy entrainer $n$-butylamine $\left(\mathrm{F}_{\mathrm{E}} / \mathrm{V}\right.$ around 0.2 ) at tray 12 in a batch extractive rectifier of 35 trays. 95 mol\% THF was obtained. A batch extractive stripper can also be used to separate the low volatility mixture THF (B)- $n$ butylamine (C) when feeding light entrainer methanol (A). Of course that later process is only relevant if the original mixture is already polluted with methanol, which unfor- 
tunately sets a $\mathrm{F}_{\mathrm{E} \text {,min }}$ limit because of the $\alpha_{\mathrm{AC}}$ occurrence. Otherwise, a classical batch azeotropic stripper is sufficient to recover $n$-butylamine.

- Diagrams 2.0-2a: two positions for the univolatility curve $\alpha_{A B}=1$ can occur. The extractive distillation is always feasible for $C$ with an indirect split. The direct split to recover A as distillate requires that the univolatility curve $\alpha_{\mathrm{AB}}=1$ intersects the AC edge.

- Diagrams 2.0-2b: two positions for the univolatility curve $\alpha_{\mathrm{AC}}=1$ can occur. The extractive distillation is always feasible for B with an indirect split and a light entrainer A. On the other hand, the direct split to recover BA as distillate requires that the univolatility curve $\alpha_{\mathrm{AC}}=1$ intersects the AB edge (class (2.0-2b)-1). Rodríguez-Donis et al. (2010) give the example of the separation of benzene (B)-cyclohexane (C) with light entrainer (A). When the light entrainer is methyl acetate one finds class $(2.0-2 b)-1$. When one uses ethyl acetate instead, class $(2.0-2 b)-2$ is obtained.

- Diagram 2.0-2c: The existence of the univolatility line $\alpha_{B C}$ sets obligatory location of $\alpha_{\mathrm{AB}}$ and $\alpha_{\mathrm{AC}}$. The feasibility criterion is only accomplished for $C$ in a region near to the zeotropic binary side $B C$.

Antipodal diagrams exist for the classes (2.0-2a), (2.0-2b) and $(2.0 .2 \mathrm{c})$ but no examples of extractive distillation have been shown so far.

- Diagram 1.0-1b heterogeneous: the heteroazeotrope is an unstable node. Thereby, heteroazeotropic distillation is feasible. Continuous entrainer feeding in a heterogeneous extractive distillation process improves product recovery (Modla et al., 2003).

- Diagram 2.0-2b heterogeneous: two positions for the univolatility curve $\alpha_{A B}=1$ can occur. The one intersecting the homogeneous zeotropic binary edge is handled by the homogeneous separation class (2.0-2b)-1 described before. If $\alpha_{A B}=1$ intersects the edge with a saddle binary heteroazeotrope (Table 5), a direct split process is feasible to obtain as distillate product the component-rich phase after liquid-liquid splitting of the heteroazeotrope. Further purification of that phase may be required.

- Diagram 2.0-2c heterogeneous: same remarks as Diagram 2.0-2b heterogeneous. No example was published so far.

- Diagram 2.1-2b heterogeneous: heavy, light and intermediate entrainers are suitable. Only the heavy case is shown in Table 5. The ternary heteroazeotrope can be either homogeneous or heterogeneous.

According to literature, the heterogeneous extractive distillation requires no rectification section to be feasible if the extractive stable node lies in the VLLE region (Modla et al., 2003; Rodríguez-Donis et al., 2007). Otherwise a rectification section is needed to reach the VLLE region in the column overhead composition.

\section{Process synthesis, design, optimization and control}

Once the process feasibility is assessed (see Section 2), the next step consists in finding a suitable column configuration and suitable operating conditions values. These are usually limited in range and concern a finite reflux or reboil ratio, and an entrainer-feed flow rate ratio that enables getting the desired product purity and recovery yield.
The models presented in this review are equilibrium ones. Only a few papers have considered nonequilibrium ratebased or efficiency-based models (Pradhan and Kannan, 2005; Junqueira et al., 2012; Gómez-Castro et al., 2015).

\subsection{Column configuration and operation mode}

Extractive distillation can be operated either in batch or in continuous mode. We can distinguish extractive distillation processes into two splits. With a direct split, one recovers a low boiling temperature product in the distillate at the extractive distillation column top and with an indirect split, one recovers a heavy boiling product at the column bottom. To be consistent with Section 2, what matters is not the product boiling point but the volatility order. Fig. 1 (see Section 1) displays typical flowsheets for the extractive distillation process for the separation of a binary mixture $A-B$ with entrainer $E$ in continuous or batch, with a heavy or a light entrainer fed at an intermediate location of the column for a direct and an indirect split. The external feeding of the entrainer at a different location than the main feed gives rise to an extractive section between the rectifying and the stripping sections for the continuous process. For the batch process, there exists no stripping section for the direct split configuration (Fig. 1c) and no rectifying section for the indirect split configuration (Fig. 1d).

For the direct split, if the entrainer is fed at the top, there is no rectifying section but still an extractive section. In that case the continuous homogeneous process is not feasible since as explained in Section 2.5, the rectifying section is needed to reach the most volatile product near a rcm saddle node. In the batch homogeneous process top entrainer feeding can be indeed optimal (e.g. Hegely and Lang, 2016) but it is usually not recommended (see Table 6). On the other hand, for the heterogeneous process, top entrainer feeding is possible and discussed in Sections 3.1.1 and 3.1.2.

The continuous process for a direct split was studied early in the twentieth century (Schneibel, 1923) and later suggested for the indirect split (Hunek et al., 1989) while batch extractive distillation was studied since the 90's (Lang et al., 1999 for the indirect split; Yatim et al., 1993 for the direct split). For the batch process many different column configurations, depending on the entrainer feed location, were investigated extensively, which results were summarized in Stéger et al. (2005) for the direct split configuration and Varga (2006) for the indirect split configuration (see Table 6 later).

\subsubsection{Continuous operating mode}

The extractive distillation process in continuous mode is assumed to operate under steady state conditions. The separation of a binary mixture $A-B$ with an entrainer $E$ requires a sequence of two connected columns, the extractive distillation column and the entrainer regeneration column (Fig. 1a direct split and $b$ indirect split). Depending on the residue curve map class and the univolatility curve location as detailed in Section 2, either A or B can be a product of the extractive distillation column. Then, the entrainer-rich stream is sent with the other non-product initial compound to the regeneration column to be recycled. In practice an entrainer make-up stream (not shown in Fig. 1) is added to compensate entrainer trace losses in the A and B-rich products. Sometimes, a more efficient process is obtained with a pre-concentrator column added to the sequence (Liang et al., 2014; An et al., 2015; Zheng et al., 2017; You et al., 2018) and alternative flowsheets can be derived when designing thermally coupled configurations 
Table 6 - Recommended configurations when using batch extractive distillation process for the separation of minimum or maximum boiling azeotrope or low relative volatility mixture with a heavy, light or intermediate boiling entrainer (Varga, 2006).

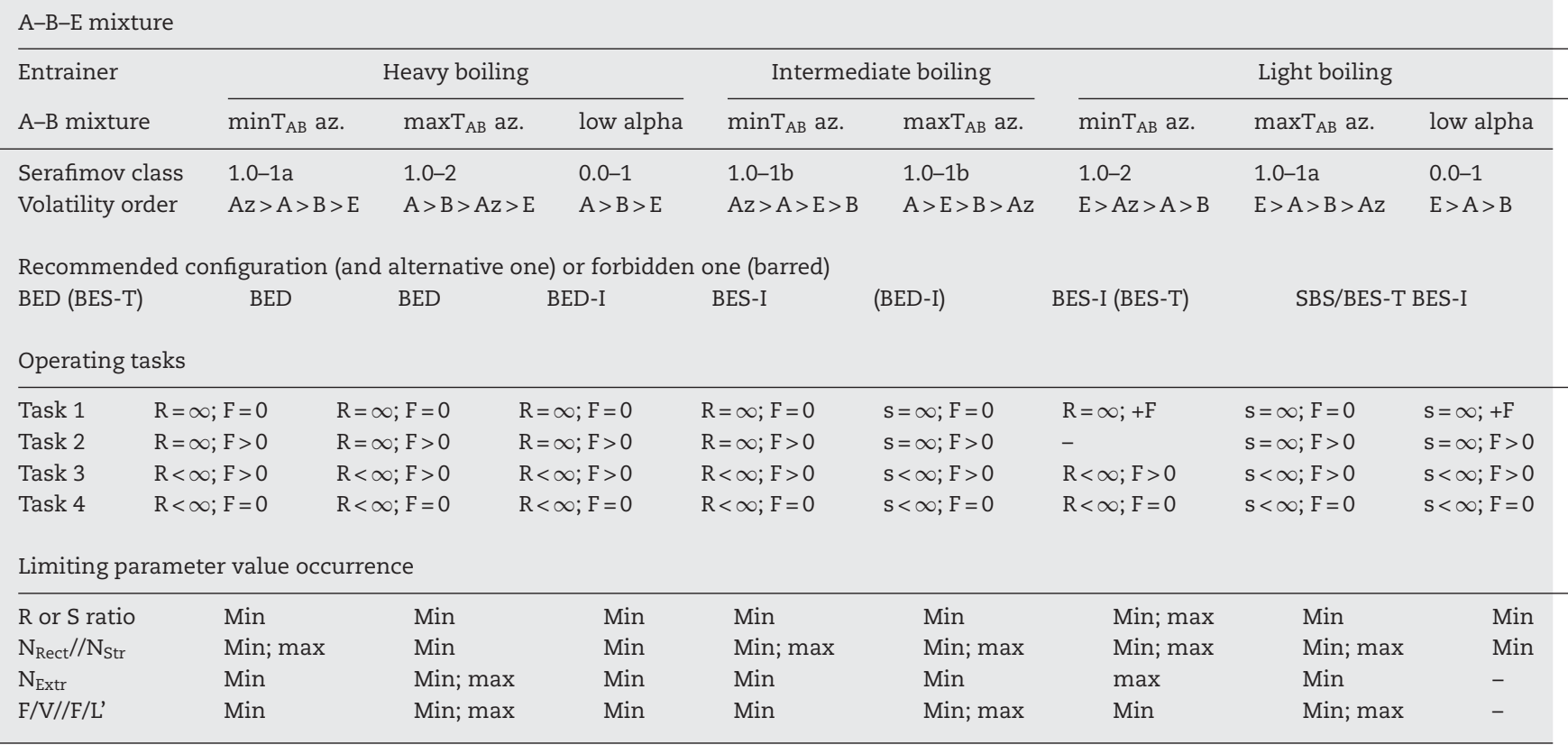

(Knight and Doherty, 1989; Laroche et al., 1991; Errico and Rong, 2012).

The most classical column sequence configurations are displayed in Fig. 1, giving rise into the extractive distillation column to three sections, rectifying, extractive and stripping ones. Commonly, a heavy entrainer $F_{E}$ is fed above the main feeding $F_{A B}$ (Fig. 1a) and a light entrainer is fed below $F_{A B}$ (Fig. 1b). Those conditions ensure a high concentration of the entrainer in the extractive section as light compound concentration increases upward and heavy compound concentration increases downward (Laroche et al., 1991). No thorough analysis has been made for the intermediate boiling entrainer feed location. The continuous column configuration with a heavy entrainer has dominated in the literature cited in the reference list, whereas the use of a light entrainer has been investigated by only a few people (Gmehling and Möllmann, 1988; Laroche et al., 1991, 1992a, 1992b; Petlyuk et al., 1999; Shen and Gerbaud, 2013; Shen et al., 2015a, 2016).

For homogeneous continuous extractive distillation operation, three configurations variants can be thought of: the entrainer can be fed with the main feed $F_{A B}$ (no extractive section, the process is then called azeotropic distillation), at another tray location or at the top of the column (no rectifying section).

As observed for the homogeneous extractive distillation separation classes reported in Tables 1-4, when the product is a saddle point (e.g. all separation classes (1.0-1a)), the feeding of the entrainer $F_{E}$ at another location than the main feed $F_{A B}$ is necessary, giving rise to an extractive section so as to allow reaching the product by means of a rectification (resp. stripping) section profile for a direct (resp. indirect) split. When the product is a stable or an unstable node (e.g. all extractive separation classes (1.0-2)), the product can be recovered by azeotropic distillation as well, with $F_{E}=0$. Therefore the entrainer feed location can be the main feed one. However, one noted that with two different locations for $F_{E}$ and for $F_{A B}$, the extractive section gives rise to a larger feasible composition region (Shen and Gerbaud, 2013).

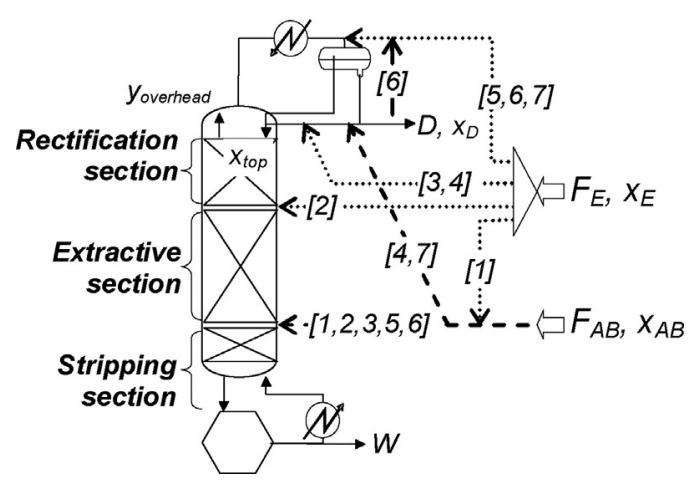

Fig. 7 - Superstructure for the heterogeneous distillation column considering seven possibilities for both the entrainer and the main azeotropic feed (adapted from Rodríguez-Donis et al., 2007. Copyright 2007 Wiley).

For heterogeneous continuous extractive distillation, there exist seven configurations summarized in Fig. 7. One considers that the main feed can be fed at an intermediate or a top location and that the entrainer feed can be fed at an intermediate or a top location or in the decanter (Rodríguez-Donis et al., 2007). Ideally, the heteroazeotrope is removed in the vapour overhead, which can be a saddle point or an unstable node (see Table 5 separation classes). Unlike the homogeneous process, the reflux composition at the top $x_{\text {top }}$ is different from the overhead vapour composition. It can be calculated by using the simplified model given in Rodríguez-Donis et al. (2007) for all seven configurations and its knowledge is necessary since the feasibility requires a continuous composition profile from $x_{\text {top }}$ to the boiler composition across the various sections of the column.

The feeding of the entrainer $F_{E}$ fed with the main feed $F_{A B}$ at an intermediate location or at the top does not provide a feasible process if the heteroazeotrope is a saddle point because of the absence of the extractive section (Rodríguez-Donis et al., 2007). The feeding of the entrainer at another location than $F_{A B}$ sets an extractive section, enabling the process to be feasible, 

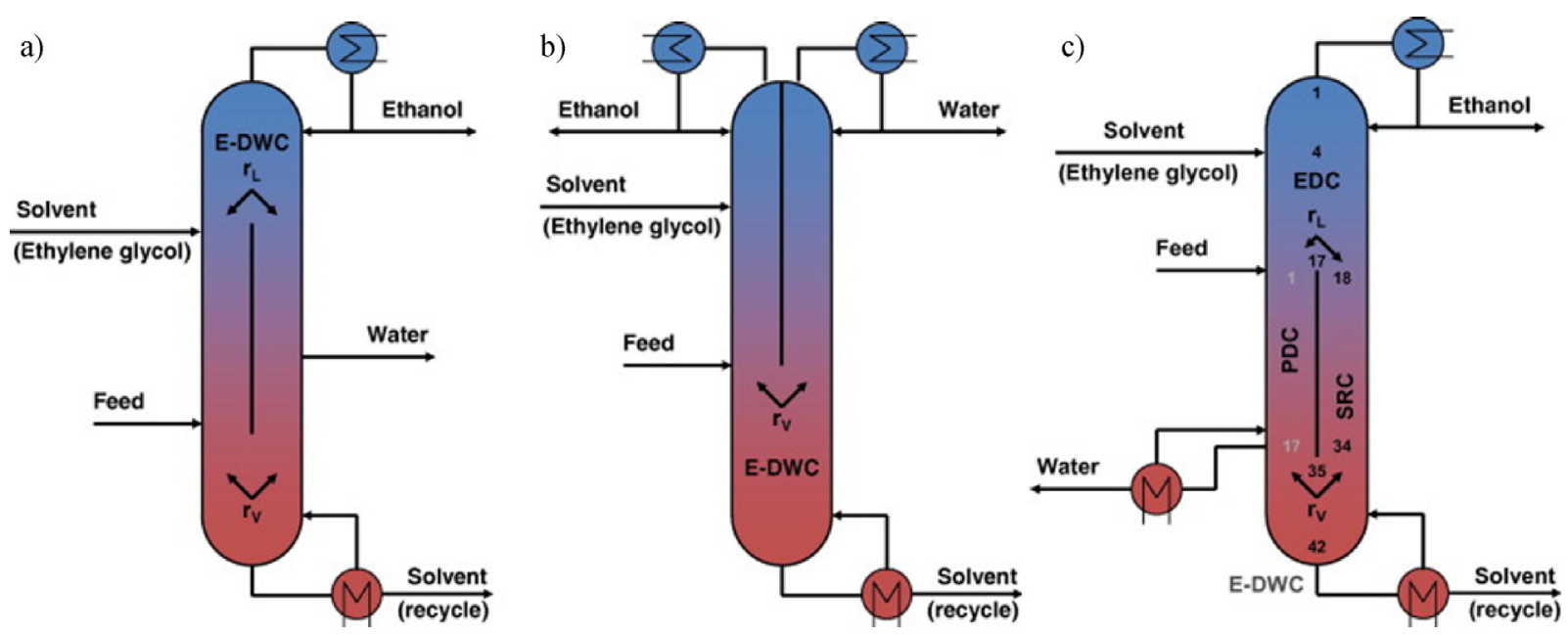

Fig. 8 - Three alternative configuration for the extractive dividing wall column (from Kiss and Ignat, 2012. Copyright 2012 Elsevier).

and the entrainer can be fed directly at the top, because the decanter can replace the rectifying section to reach the distillate product. In some cases, feeding the entrainer into the decanter or recycling of a portion of the distillate is necessary to get a more favourable liquid-liquid split ratio. If the extractive section stable node approaches the heteroazeotrope, the rectification section is not necessary for the process to be feasible.

The choice of the physical state of the entrainer stream, e.g. a boiling liquid or a saturated vapour, has been discussed by some authors. For heavy entrainers, Knapp and Doherty (1994) recommended a subcooled entrainer feed and stated that a partially vaporized main feed reduces the contribution of the entrainer feed to the internal reflux in the extractive section, thereby inducing a larger external reflux to the column to achieve the same separation. For light entrainers, similar consideration would suggest to feed a saturated vapour entrainer feed below the main feed location (Shen and Gerbaud, 2013b).

Other special configurations have been proposed, like column with partial condenser (Taylor and Wankat, 2004), extractive column with a side stream (Han et al., 2015), thermal coupling between the extractive and entrainer regeneration columns (Errico and Rong, 2012; You et al., 2016b), thermal integration secondary reflux and vaporization (Batista and Meirelles, 1997), heat integrated distillation column (HIDiC) (Jana, 2010; Kiss and Olujić, 2014), side-stream extractive distillation (Tututi-Avila et al., 2017), Peyluk column with complex arrangement (Timoshenko et al., 2005; Gutiérrez-Guerra et al., 2009) and extractive dividing-wall column (Bravo-Bravo et al., 2010), merging the pre-concentrator column with the extractive column (An et al., 2015) or the entrainer regeneration column (Liang et al., 2014; Dongmin and Yanhong, 2018). The lists is not exhaustive. Kiss and Ignat (2012) summarized three alternatives for the extractive dividing-wall column (E-DWC) with heavy entrainer displayed in Fig. 8.

The first alternative (Fig. 8a) is a direct adaptation from usual dividing wall column (Bravo-Bravo et al., 2010). Modla (2013) demonstrated that this configuration is feasible for intermediate boiling entrainer, when the entrainer is obtained as the side product. The second alternative (Fig. 8b) is the most used in the literature (Midori et al., 2000; Gutiérrez-Guerra et al., 2009; Yildirim et al., 2011; Kiss and Suszwalak, 2012; Kiss and Ignat, 2012; Xia et al., 2012; Wu et al., 2013; Sun et al., 2014; Zhang et al., 2014; Huang et al., 2016). It combines the conventional extractive column and the regeneration column in a single shell with one boiler and two condensers. Compared to alternative "a" (Fig. 8a), there is no liquid split as the inner wall goes to the top. On the left side, there is a rectifying section and an extractive section. The stripping section is below the wall and is common to the right side which mimics the regeneration column. Feasibility of that configuration is assessed in the same way than in Section 2. However, the vapour split ratio is an additional parameter to be set. Most of the literature on E-DWC use this configuration. The third configuration has a single condenser and two reboilers (Kiss and Ignat, 2012; Luo et al., 2015). It was proposed after noting that the second configuration required to vaporize water to the top, which required energy, and allowed double digits energy savings (Kiss and Ignat, 2012) that could be enhanced by combining it with vapour recompression (Luo et al., 2015; Patrascu et al., 2017; Patrascu and Bildea, 2017; Luyben, 2017). Other EDWC configurations have been studied with a side decanter for heterogeneous mixtures, but with the wall located below the usual extractive section (Yang et al., 2018).

\subsubsection{Batch operating mode}

Extractive batch distillation operates under unsteady state conditions, with all compositions varying with time. It is a semi-batch process, as the main feed $F_{A B}$ is loaded initially in the boiler, whereas the entrainer is fed continuously at a higher tray. The entrainer regeneration task is performed in the same column.

Typically, for a direct split, after loading the main feed into the boiler, the product will be removed as distillate from a column with a rectification section above the extractive section (Fig. 1c). For an indirect split, after splitting the main feed load between a small boiler and a large top vessel tank, the product will be removed from the bottom from a column with a stripping section below the extractive section (Fig. 1d).

The batch extractive distillation process runs in four steps (Yatim et al., 1993). Firstly, after the initial charge is loaded, the process is operated with no entrainer feeding and no product removal. Heating starts and proceeds until steady state is achieved and the azeotrope is obtained at the top (direct split) or at the bottom (indirect split). Secondly, the entrainer is fed continuously so as to quickly substitute the azeotropic product by a high purity compound. The dynamic of that substitution is usually rapid and readily observed by a shift 


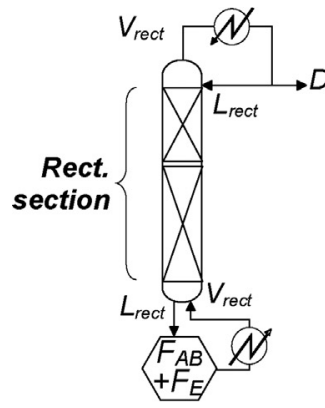

(a) SBD

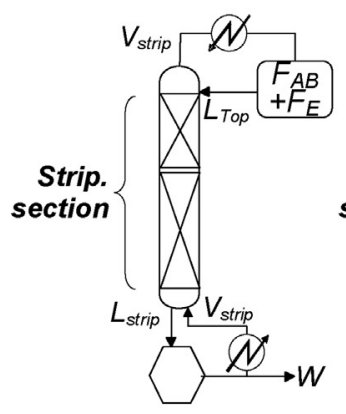

(e) SBS

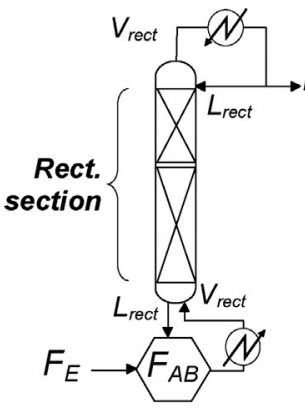

(b) BED-B

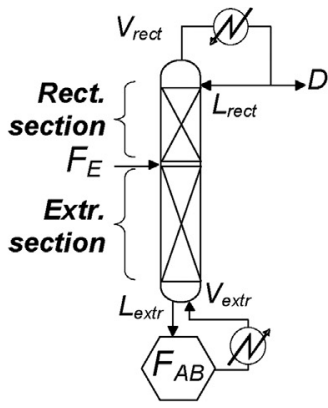

(c) BED-I

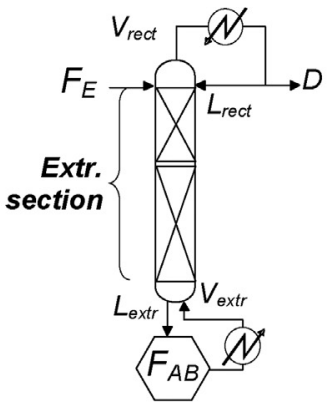

(d) BED-T

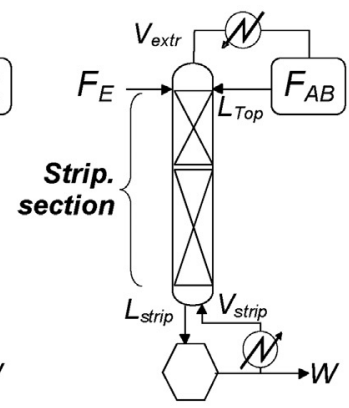

(f) BES-T

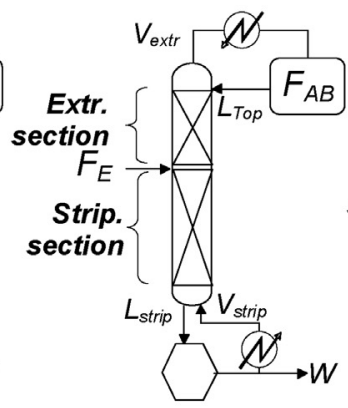

(g) BES-I

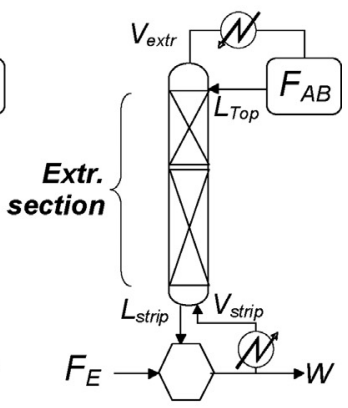

(h) BES-B

Fig. 9 - Column configurations with different feed locations for batch homogeneous extractive distillation processes.

from the column top temperature from the azeotrope temperature to the pure compound temperature. Thirdly, a product is removed under steady entrainer feeding. For the final step, entrainer is no longer fed and a classical distillation allows the entrainer accumulation into the boiler while distillation of the remaining original compound is performed. The first and second steps can sometimes be combined as it was shown that energy and time could be saved when the entrainer feeding is started before the steady state is attained. Entrainer feeding during the first step prevents the condensate composition from approaching that of the azeotrope, thus the desired distillate purity at the end of the second step is reached faster (Lang et al., 2006). This was tested with dynamic simulations of the batch extractive process under the assumption of a simple start-up period scheme (tray by tray filling, no internal reflux) (Lang et al., 1994; Lelkes et al., 1998b; Milani, 1999; Lang et al., 2006) and a more detailed start-up period model (Repke et al., 2007).

Several authors investigated alternative operation policies of batch extractive distillation. The batch extractive distillation modified policy (BEDM), suggested by Lang et al. (2006) consists in starting to feed the entrainer already during the start-up (Lang et al., 2012; Hegely, 2013). In this way, it is possible to extract impurities before their concentration reaches its high, steady-state value, and to decrease the duration of the heating-up. Another batch extractive distillation policy (BED1, Lang et al., 2012), consists in feeding the entrainer only during the heating-up of the column, and the entrainer extracts the main component after the polluting components, removed in the fore-cuts.

Thanks to its dynamic nature, batch extractive distillation offers more feasible column configuration alternatives than the continuous process. Several authors have studied rectifying and stripping columns with four different entrainer feeding locations shown in Fig. 9: (option 1) initially the heavy, light or intermediate boiling entrainer is fed with the azeotropic mixture into the still (resp. top tank) for rectifying (resp. stripping) column (Simple Batch Distillation SBD (resp. Simple Batch Stripper SBS) process), (option 2) continuously into the main tank (Batch Extractive Distillation - Bottom BEDB (resp. Batch Extractive Stripper - Top BES-T) process), (option 3) at an intermediate point of the column (BED-I (resp. BES-I) process) and (option 4) at the column top (resp. bottom) for rectifying (resp. stripping) column (BED-T (resp. BES-B) process). Therefore, the column has a single rectifying (resp. stripping) section for rectifying (resp. stripping) column (option 1 and 2) or an extractive section (option 4) or two sections, rectifying (resp. stripping) and extractive one, for rectifying (resp. stripping) column (option 2). Using the differential model (Van Dongen and Doherty, 1985a; Lelkes et al., 1998a) for a rectifier column and for a stripper column (Varga, 2006; Shen and Gerbaud, 2013b), those authors systematically calculated composition profile maps in each column section under various process operating conditions. They were able to assess the feasibility of all eight column configurations for the separation of minimum or maximum boiling azeotropes and close-boiling mixtures and to evaluate the occurrence of limiting values for the reflux, for the (entrainer feed/vapour flowrate) ratio, and for the number of stages in the various column sections (Stéger et al., 2005; Varga, 2006). Comparison of all processes leads to recommend the use of BED-I with a heavy entrainer. With a light entrainer the BES-I column configuration was recommended (Lang et al., 1999; Varga, 2006; Shen et al., 2015c) but a batch rectifier configuration is also possible (Stéger et al., 2005; Varga et al., 2006a, 2006b). Table 6 summarizes the configurations recommended when using batch extractive distillation process for the separation of minimum or maximum boiling azeotrope or low relative volatility mixtures with a heavy, light or intermediate boiling entrainer (Varga, 2006)

With a heavy entrainer, the main load $F_{A B}$ is initially in the still, the batch column is a rectifier with an extractive and a rectifying section (Fig. 1c); the product is removed as distillate from the top and the separation is a direct split. The operating parameters are the entrainer-vapour flow rate ratio of $F_{E} / V$, 
the reflux ratio $(R=L / D)$ established at the column top and the number of equilibrium trays in each column section. $F_{E} / V$ is an entrainer-feed flow rate ratio considering that $F_{A B}$ is fed into the column as a vapour overflow $V$ from the boiler.

With a light entrainer, the initial load is split between the top vessel and the boiler. The batch column is a stripper with an extractive and a stripping section; the product is removed as bottom product and the separation is an indirect split. The key operating variables are the entrainer-top liquid flow rate ratio $F_{E} / L_{T}$, the bottom reboil ratio $S\left(S=V_{S} / W\right)$ and the number of equilibrium trays in each column section. $F_{E} / L_{T}$ is an entrainer-feed flow rate ratio considering that $F_{A B}$ is fed to the column top through the liquid flow rate $\left(L_{T}\right)$ coming from the top vessel (Fig. 1d).

Other more sophisticated configurations of the batch extractive distillation process have been proposed: batch extractive middle vessel column (Safrit and Westerberg, 1997a,b; Safrit et al. 1995; Hilmen et al., 1997; Warter and Stichlmair, 1999; Low and Sorensen, 2002; Cui et al., 2002; Kim et al., 2004; Warter and Stichlmair, 1999, 2004; Kotai and Lang, 2005; Stéger, 2006; Li et al., 2006), heterogeneous batch extractive distillation (Modla et al., 2003; RodríguezDonis et al., 2003, 2007; Van Kaam et al., 2008), or double boiler batch column configurations (Hua et al., 2007). In theory, they bring under certain conditions some advantages in terms of recovery, but to our knowledge, none is currently operated in industry, possibly because of their lower flexibility compared to usual configurations. Regarding heterogeneous extractive distillation, feeding the entrainer at the top is usually relevant, because the top decanter can help reach the distillate product without the need of a rectifying section (Van Kaam et al., 2008).

\subsection{Operating parameters for extractive distillation with intermediate entrainer feeding}

From now, we consider the column configurations shown in Fig. 1, with the entrainer fed at an intermediate tray location, inducing that there exists a so-called extractive section in the column. The reflux ratio $R$ is a key operating parameter for a direct split separation with intermediate entrainer feeding (Fig. 1a and c). It is defined by the ratio of the liquid reflux flow rate entering the rectifying section and the distillate product flow rate $D$ :

$R=\frac{L_{r e c t}}{D}$

The reboil ratio $S$ is a key operating parameter for an indirect split separation (Fig. $1 \mathrm{~b}$ and d). It is defined by the ratio of the vapour flow rate entering the stripping section and the bottom product flow rate $\mathrm{W}$ :

$S=\frac{V_{\text {strip }}}{W}$

Regarding the entrainer-feed flow rate ratio, there can be several definitions, depending on the operating mode and the type of separation split:

- For a continuous extractive distillation process, it is defined for both direct and indirect splits as $F_{E} / F_{A B}$, the ratio of the external entrainer feed, $F_{E}$ and the external main feed, $F_{A B}$ (Fig. 1a and b).
- For a batch extractive distillation process, only the entrainer is fed externally to the column. The main feed is given by the vapour flow rate $V_{\text {extr }}$, entering the extractive section from the boiler, for the direct split separation (Fig. 1c) and by the top liquid flow rate $L_{T o p}$, refluxed to the column from a top vessel and entering the extractive section, for the indirect split separation (Fig. 1d). Then the entrainer-feed flow rate ratio in batch is defined as $F_{E} / V_{\text {Extr }}$ for the direct split and as $F_{E} / L_{T o p}$ for the indirect split.

For the sake of comparing operating conditions for the batch and the continuous processes, one would like to transform limiting values of the entrainer-feed flow rate ratio from batch to continuous operation. For that purpose, one can derive the following relations between the continuous value $F_{E} / F_{A B}$ and the batch values $F_{E} / V_{\text {extr }}$, or $F_{E} / L_{T o p}$ (Shen et al., 2013; Shen and Gerbaud, 2013).

$\left(\frac{F_{E}}{F_{A B}}\right)=(R+1) \cdot\left(\frac{F_{E}}{V_{\text {extr }}}\right) \cdot\left(\frac{D}{F_{A B}}\right)$

where both feeds are considered as a boiling point liquid.

$\left(\frac{F_{E}}{F_{A B}}\right)=\left(\frac{F_{E}}{L_{T o p}}\right) \cdot\left((S+1) \cdot \frac{W}{F_{A B}}-1\right) /\left(1+q \cdot\left(\frac{F_{E}}{L_{T o p}}\right)\right)$

where $q=0$ for a saturated vapour entrainer feed and $q=1$ for a boiling liquid entrainer feed. The main feed is fed as a boiling liquid.

\subsection{Finding suitable operating parameter conditions}

To guide engineers designing extractive distillation processes, there exist several simplified models based on the following assumptions: a column operation mode and configuration is chosen; the product purity and recovery target values are fixed, constant molar overflow and negligible tray holdup is assumed.

Then, for a given set of the operating parameters cited above, the feasibility assessment methodology is based on the analysis of the liquid composition $\times$ profile computed for each of the extractive column stripping, extractive and rectifying sections. The process is deemed feasible if they intersect each other and connect the top liquid composition to the bottom liquid composition of the column. The top and bottom compositions depend on the column configuration and on the reflux policy for heterogeneous extractive distillation (Rodríguez-Donis et al., 2003, 2007; Van Kaam et al., 2008). In batch mode, Hegely et al. (2014) proposed a generalised model for studying feasibility of heterogeneous extractive batch distillation.

\subsubsection{Defining suitable product recovery yields and} recycle entrainer purity

Consider the continuous extractive distillation process for a direct split (Fig. 1a). In many papers, the choice of both distillate flow rates $D_{1}$ and $D_{2}$ seems arbitrary, but some authors noticed that certain operating conditions were incompatible with achieving a high purity and recovery target for A whatever the heat duty (Luo et al., 2014) or a high purity for both A and B products (Gil et al., 2009). However, owing to the entrainer recycle stream, $D_{1}$ and $D_{2}$ flow rates are strongly interrelated with each other. Only recently You et al. (2016a) showed that an unreasonable choice of distillate flow rate may 
Table 7 - Relationship between distillates, purity and recovery for $100 \mathrm{kmol} / \mathrm{h}$ binary mixture (You et al., 2016a with permission from Elsevier).

Feed composition Purity specification

$\begin{array}{llll}\mathrm{x}_{1} & 0.75 & \mathrm{x}_{\mathrm{D} 1, \mathrm{~A}} & 0.995 \\ \mathrm{x}_{2} & 0.25 & \mathrm{x}_{\mathrm{D} 2, \mathrm{~B}} & 0.995\end{array}$

Range of distillate (Eqs. (10) and (11))

$\mathrm{D}_{1} / \mathrm{kmol} / \mathrm{h}$ (Eq. (10)) $75.00\left(\Psi_{\mathrm{A}}=99.5 \%\right) \quad 75.37\left(\Psi_{\mathrm{A}}=100 \%\right)$

$\mathrm{D}_{2} / \mathrm{kmol} / \mathrm{h}$ (Eq. (11)) $\quad 25.00\left(\Psi_{B}=99.5 \%\right) \quad 25.12\left(\Psi_{B}=100 \%\right)$

Range of distillate (Eqs. (12) and (13))

$\mathrm{D}_{1} / \mathrm{kmol} / \mathrm{h}$ (Eq. (13)) $>75.37\left(\Psi_{B}=98.493 \%\right) \quad>75.00\left(\Psi_{B}=98.50 \%\right)$ $\mathrm{D}_{2} / \mathrm{kmol} / \mathrm{h}$ (Eq. (12)) $\quad>25.12\left(\Psi_{\mathrm{A}}=99.8325 \%\right) \quad>25.00\left(\Psi_{\mathrm{A}}=99.833 \%\right)$

lead to poor product quality and/or enhance the difficulty of the separation, requiring more energy and cost to achieve the same product recovery. They also showed that it sets limits on the entrainer recycle stream and its impurity content, and that looking for a higher recovery yield may cost sometimes less energy. Here is now their derivation.

For a binary azeotropic mixture feed $F_{A B}$ separated in a direct split process configuration (Fig. 1a), the product purities $x_{D 1, A}, x_{D 2, B}$ and process recoveries $\Psi A, \Psi B$, obey the relations

$$
\begin{aligned}
& A=\frac{D_{1} x_{D 1, A}}{F x_{F, A}} \rightarrow D_{1}=\frac{F x_{F, A} \psi_{A}}{x_{D 1, A}} \\
& B=\frac{D_{2} x_{D 2, B}}{F x_{F, B}} \rightarrow D_{2}=\frac{F x_{F, B} \psi_{B}}{x_{D 2, B}}
\end{aligned}
$$

But we can also write the mass balance for A (Eq. (12)) and B (Eq. (13)) and use Eqs. (10) and (11) to obtain the influence of one component's recovery on the other distillate flow rate:

$$
\begin{aligned}
& D_{2}=\frac{F x_{F, A}-D_{1} x_{D 1, A}}{1-x_{D 2, B}-x_{D 2, E}}=\frac{F x_{F, A}\left(1-\psi_{A}\right)}{1-x_{D 2, B}-x_{D 2, E}}>\frac{F x_{F, A}\left(1-\psi_{A}\right)}{1-x_{D 2, B}} \\
& D_{1}=\frac{F x_{F, B}-D_{2} x_{D 2, B}}{1-x_{D 1, A}-x_{D 1, E}}=\frac{F x_{F, B}\left(1-\psi_{B}\right)}{1-x_{D 1, A}-x_{D 1, E}}>\frac{F x_{F, B}\left(1-\psi_{B}\right)}{1-x_{D 1, A}}
\end{aligned}
$$

where $x_{D 1, E}$ and $x_{D 2, E}$ are the entrainer contents in the two distillates.

From these equations we observe that the distillate flow rate $D_{2}$ (resp. $D_{1}$ ) is controlled by the recovery and product purity of B (resp. A), and by the recovery of A (resp. B). Hence, an unreasonable choice of distillate flow rate may lead to poor product quality and/or enhance the difficulty of the separation, requiring more energy and higher cost to achieve the same product recovery.

You et al. (2016a) applied these equations to the separation of the system diisopropyl ether (DIPE)-isopropanol (IPA) with 2-methoxyethanol as entrainer. The results are shown in Table 7 below.

From Table 7, we know that (1) following Eqs. (10) and (11), the value range of A-rich distillate $D_{1}$ and B-rich distillate $D_{2}$ is easy to calculate from $\Psi_{\mathrm{A}}$ and $\Psi_{\mathrm{B}}$ respectively, but it is not strict enough. For example, component A's recovery $\Psi_{\text {A }}$ (see Eq. (12)) has to be within a very narrow value range around $99.832 \%$. Otherwise $D_{2}$ can not reach the $\{25-25.51\} \mathrm{kmol} / \mathrm{h}$ range that is expected. Likewise for $\Psi_{B}$ (see Eq. (13)). Therefore, for a binary mixture of $A B$ with $100 \mathrm{kmol} / \mathrm{h}$ and $x_{F, A}=0.75$, if the two products specifications are set at $98 \%$, we should choose
$\Psi_{A}$ between [99.3197\%, 99.333\%], which will ensure that $\Psi_{B}$ is within $[98 \%, 100 \%]$

These relations also indicate that the choice of a distillate flow rate impacts the necessary entrainer feed flow rate and its impurity content. For example, if we assume $D_{1}=75 \mathrm{kmol} / \mathrm{h}$, equation 10 gives $\Psi_{A}=0.98$ for a purity of $x_{D 1, A}=0.98$. Thus there will be $75 \times(1-0.98)=1.5 \mathrm{kmol} / \mathrm{h}$ of A entering the second regeneration column. As $D_{2}=25 \mathrm{kmol} / \mathrm{h}$, the purity of $B$ will be not higher than $x_{D 2, B}=(1-1.5 / 25)=0.94$ if we neglect the loss of $A$ in the recycled entrainer stream $W_{2}$. On the other hand, if we want to achieve $x_{D 2, B}=0.98$, the maximum value of $A$ that should leave in $D_{2}$ is limited to $0.5 \mathrm{kmol} / \mathrm{h}$ $(25 \times(1-0.98))$. Subtracted from the $1.5 \mathrm{kmol} / \mathrm{h}$ entering the regeneration column, there remains $1 \mathrm{kmol} / \mathrm{h}$ A to be recycled at most with the entrainer recycle stream $\mathrm{W}_{2}$. That impurity in the entrainer recycle implies that the entrainer flow rate has to be over $1000 \times(1 \div(1-0.999)) \mathrm{kmol} / \mathrm{h}$ in order to guarantee our specification of $99.9 \mathrm{~mol} \%$ pure entrainer recycled to the extractive column. This corresponds to a very large entrainerto-feed flow rate ratio $\left(F_{E} / F=10\right)$, leading to a large energy cost. A more reasonable choice would then be to increase $D_{1}$ from 75 up to $76.1 \mathrm{kmol} / \mathrm{h}$. Then with $x_{D 1, A}=0.98 \mathrm{Eq}$. (1) gives $\Psi_{\mathrm{A}}=0.99437$ and $76.1 \times(1-0.0 .99437)=0.428 \mathrm{kmol} / \mathrm{h}$ of A would enter the second column. Being below the maximum $0.5 \mathrm{kmol} / \mathrm{h}$ of $\mathrm{A}$ to enter the regeneration column, the purities of $B$ and recycling entrainer can be met and the entrainer-tofeed flow rate ratio does not have to be very high, which could result in energy cost savings.

\subsubsection{Reflux or reboil ratio and entrainer-feed flow rate ratio}

The classical operating parameters are the entrainer-feed flow rate ratio $\left(F_{E} / V\right.$ for batch direct split; $F_{E} / L_{T}$ for batch indirect split; $F_{E} / F$ for continuous direct or indirect split), the top reflux ratio $\left(R=L / D\right.$, direct split) or the bottom reboil ratio $S\left(S=V_{S} / W\right.$, indirect split). In addition to that, one can consider the pressure and the number of equilibrium trays in each column section.

In Section 2, feasible thermodynamic ternary diagrams A-B-E were derived from the general feasibility criterion under infinite reflux. Analysis of each extractive separation class hinted at the occurrence of limiting values of the entrainer flow rate $\left(F_{E}\right)$, either minimum or maximum, from the intercept point of the univolatility line $\alpha_{\mathrm{AB}}=1$ with the ternary diagram edges (see Tables 1-5). An important change occurs for finite reflux ratio values as the feasible region in the composition space is reduced because of the movements of the extractive composition profile map stationary points into the composition triangle (see Fig. 5e and $\mathrm{f}$ in Section 2.4). Fig. 10 displays diagrams for the typical $\mathrm{minT}$ azeotrope extractive separation with a heavy entrainer (1.0-1a-m1 class) in a continuous distillation column.

Fig. 10a assesses the feasibility for $R=10$. The proposed entrainer-feed flow rate ratio value is $F_{E} / F=0.858$ (or equiv. $F_{E} / V=0.153$ ). These values depend on the thermodynamic model chosen, which sets the location of the univolatility curve. The operating conditions with a large reflux ratio set the extractive section stable node on the binary edge water-acetone near its intersection with the univolatility curve. Hence, extractive profiles, approximated by differential equations that will be established in the next Section 3.3.3 can intersect a rectifying section profile that reaches the expected distillate purity $x_{D A}$. Fig. $10 \mathrm{~b}$ shows steady state simulation results for a continuous column. For $R=10$ and $F_{E} / F=0.858$, 


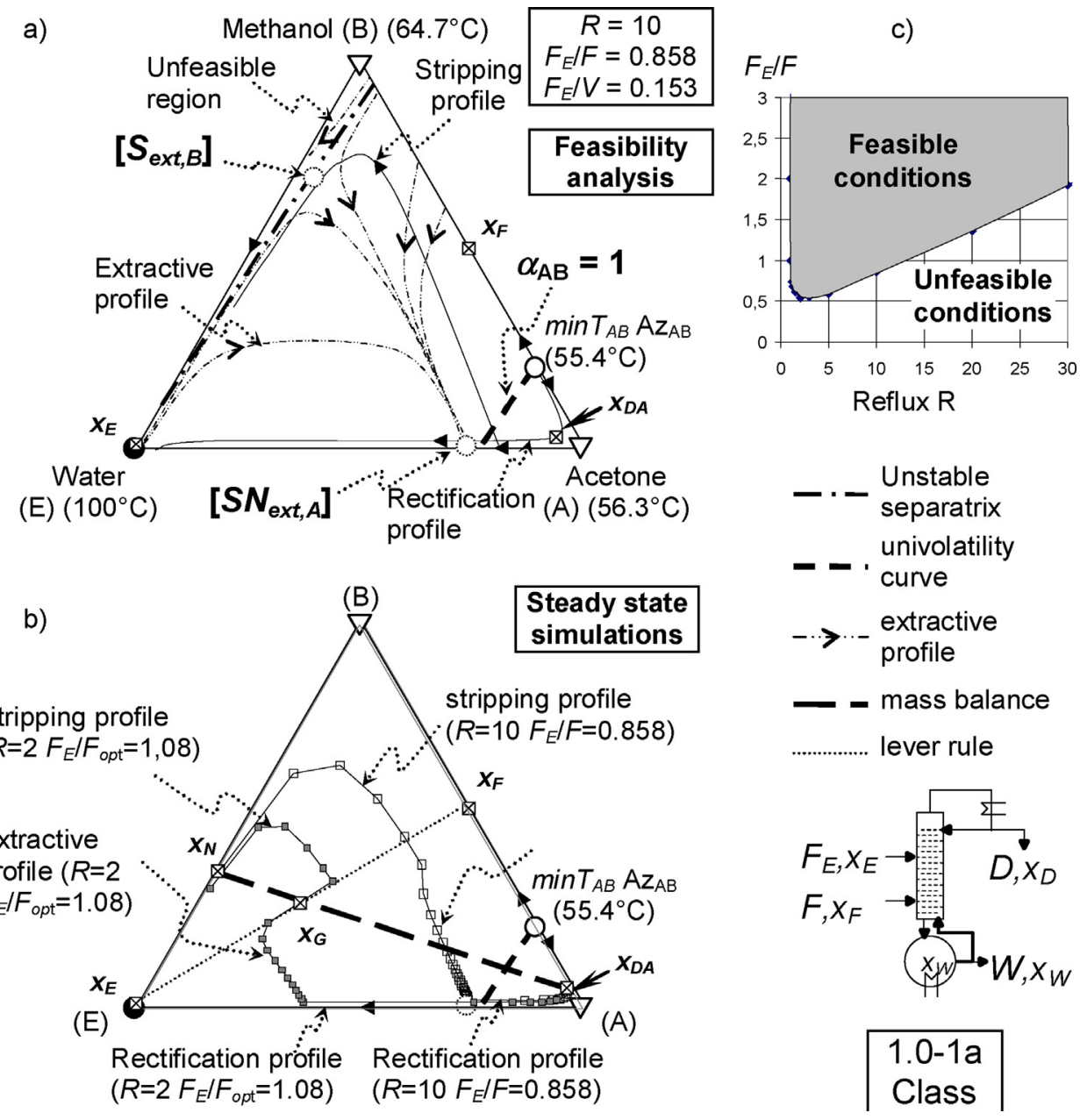

Fig. 10 - Feasibility diagram for the 1.0-1a extractive separation class, range of feasible parameters and simulation.

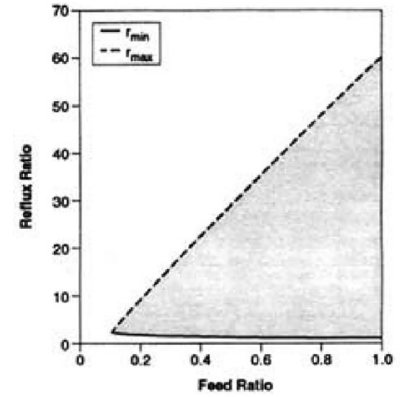

(a)

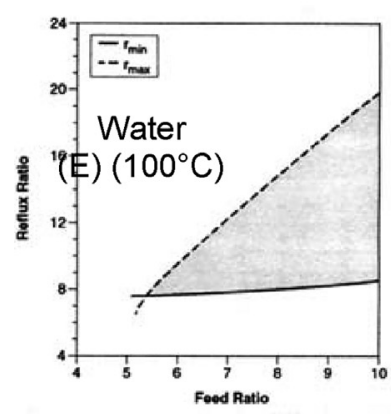

(c)

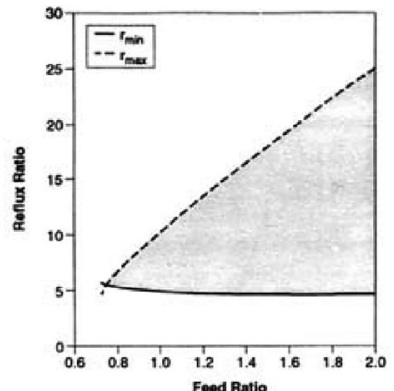

(b)

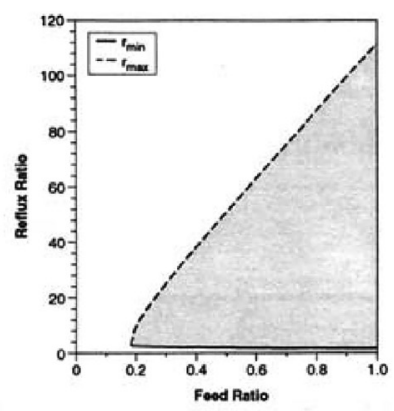

(d)

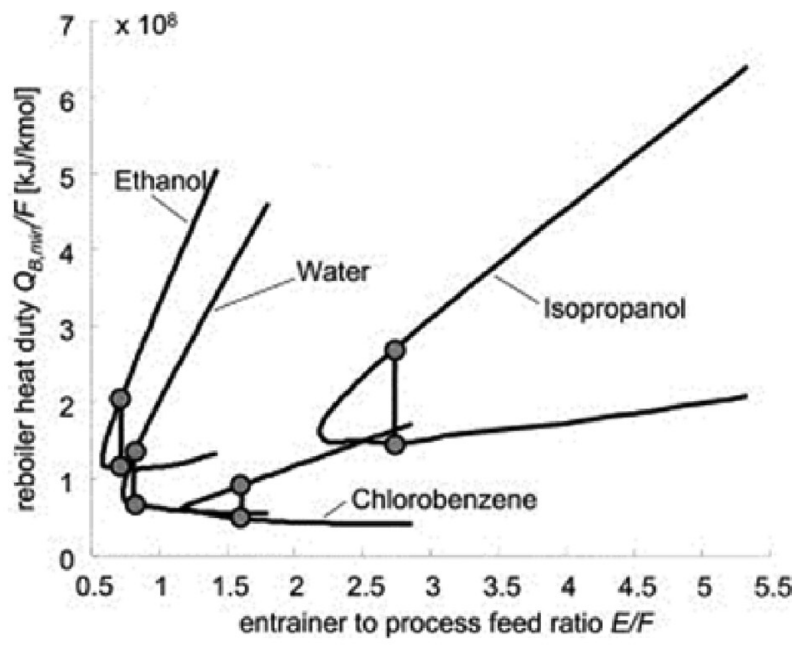

Fig. 11 - 1.0-1a extractive separation class, range of feasible parameters (left from Knapp and Doherty, 1994; right from Brüggemann and Marquardt, 2004, both with permission from Wiley).

all three column section profiles match closely the approximate profiles of Fig. 10a. Better conditions can be found: $R=2$ and $F_{E} / F=1.08$. The slight increase of operating cost due to the increase in entrainer flow rate is readily compensated by the important cost reduction thanks to a lower reflux ratio. A systematic study allows finding the range of feasible conditions 


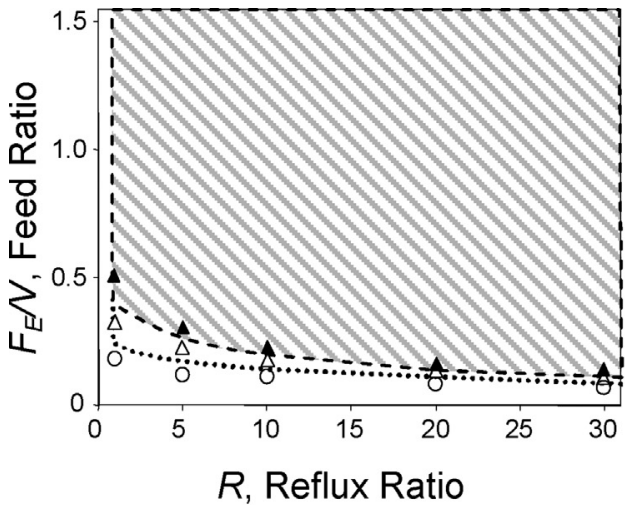

..... Continuous feasible line sketch

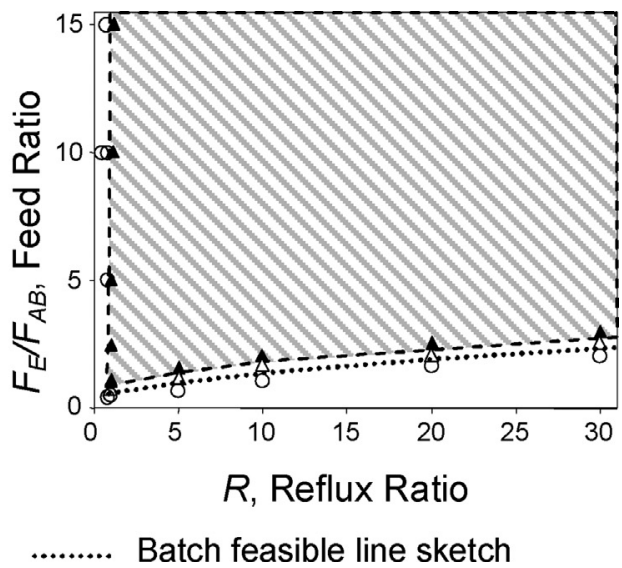

Batch feasible line sketch

Fig. 12 - Feasible ranges of entrainer-feed and reflux ratios expressed (a) in batch variable (b) in continuous variable for the batch and continuous extractive distillation of acetone-heptane with toluene (1.0-1a)-m1 class (adapted with permission from Shen et al., 2013. Copyright 2013 American Chemical Society).

for reflux and entrainer-feed flow rate ratios (see Fig. 10c). Fig. 11 also displays minimum and maximum reflux ratio as a function of entrainer-feed flow rate ratio for other $1.0-1 \mathrm{a}$ extractive separation class systems.

Fig. 11 on the left refers to (a) acetone-methanol-water separation; (b) acetone-methanol-MEK separation; (c) methanol-MEK-sec-butanol separation; (d) ethanol-water-ethylene glycol separation (Knapp and Doherty, 1994). Fig. 11 on the right refers to the acetone-methanol separation with four entrainers expressed in terms of boiler heat duty, which is related to the reflux ratio (Brüggemann and Marquardt, 2004). For engineers' practice, these two works also prescribe operating ratios. For example, in Fig. 11 right, the grey dots denote $\mathrm{Q}_{\mathrm{B}, \min }$ and $\mathrm{Q}_{\mathrm{B}, \max }$ at two operational ratios prescribed by the authors: $F_{E} / F_{E \min }=1.1$ and $R_{\max } / R_{\min }=2.0$. Alternatively, Knapp and Doherty (1994) prescribed to use the classical value $R_{\text {operation }} / R_{\min }=1.2-1.5$ and found empirically that $F_{E \text {,operation }} / F_{E \text { min }}=2.0-4.0$ corresponded to a design within "10-15\% of the lowest cost design".

The choice of a suitable entrainer flow rate is critical as it impacts evidently the economics and the controllability of the process. For example, the dynamic control performance could be improved by increasing slightly the entrainer flow rate above the value found for the design with the lowest TAC (Wang et al., 2015b).

In batch operation, the feasible range of parameters entrainer-feed flow rate and reflux ratios seen in Figs. 10 and 11 are larger than the continuous ones (Shen, 2012; Shen et al., 2013). For a fixed $R$, the batch process is feasible under a smaller entrainer-feed flow rate ratio as seen in Fig. 12: the same entrainer-feed flow rate ratio vs reflux ratio plot is displayed in batch mode parameter $F_{E} / V$ on the left and in continuous mode parameter $F_{E} / F$ on the right. This happens because of the additional condition for the continuous process that the stripping and the extractive section profiles intersect each other.

Unsurprisingly, the shape of the feasible region is dissimilar for the batch and the continuous mode. As Eq. (8) shows that when $\left(F_{E} / V\right)$ is fixed, $\left(F_{E} / F_{A B}\right)$ depends on $R+1$ but also on $D / F_{A B}$.

\subsubsection{Intersection of composition profiles}

Early studies extended methods developed for single feed azeotropic distillation columns (Levy et al., 1985) to doublefeed columns (Levy and Doherty, 1986; Wahnschafft and Westerberg, 1993) for the analysis of extractive distillation pro- cesses by looking at the composition profiles in each column section. The finding of pinch points for each section profiles allowed determining the limiting values of the operating parameters.

Levy and coworkers' works relied upon plate by plate calculations, leading to discrete profiles whose segments matched equilibrium tray composition vectors in each section. Earlier, a differential approach was proposed by Acrivos and Amundson (1955), derived again by Van Dongen and Doherty (1985a) and used by Lelkes et al. (1998a) for batch extractive distillation.

The model is based on the following simplifying assumptions: (1) theoretical plates; (2) saturated liquid feed; (3) constant molar flow rates of liquid in the three respective sections of the column; (4) constant molar vapour flow rate throughout the column, (5) the liquid is an incompressible fluid. Note that the relaxation of hypothesis (4) can be done for non-negligible heat effects (Knight and Doherty, 1986).

Assuming that the main feed is a boiling liquid, the differential equations are:

\subsubsection{Stripping section.}

$\frac{d x_{i}}{d h}=\left(\frac{S}{S+1}\right)\left[\left(1+\frac{1}{S}\right) x_{i}-\frac{1}{S} x_{W}-y_{i}^{*}\right] \quad i=1, n c$

$y_{i}^{*}$ is the concentration of compound $i$ in the vapour in equilibrium with $x_{i} \cdot y_{i}{ }^{*}$ is computed by using a proper thermodynamic model.

3.3.3.2. Extractive section. For a direct split with a boiling liquid entrainer $F_{E}(q=1)$, the equation for continuous mode is derived from a top envelope mass balance, in terms of reflux ratio $R$ and the entrainer-feed flow rate ratio $F_{E} / F_{A B}$ :

$$
\begin{aligned}
& \frac{d x_{i}}{d h}=\frac{R+1}{R+\left(\frac{F_{E}}{D}\right)}\left[\left(\frac{R}{R+1}+\frac{1}{R+1}\left(\frac{F_{E}}{D}\right)\right) x_{i}\right. \\
& \left.+\frac{1}{R+1} x_{D}-\frac{1}{R+1}\left(\frac{F_{E}}{D}\right) x_{E}-y_{i}^{*}\right] \quad i=1, n c
\end{aligned}
$$

$R$ is the reflux ratio equal to $\mathrm{L}_{\mathrm{Top}} / \mathrm{D}$ (see Fig. 1 notation). By using Eq. (8), this expression is equivalent to the one for batch distillation (Van Dongen and Doherty, 1985a): 


$$
\begin{gathered}
\frac{d x_{i}}{d h}=\frac{R+1}{R+(R+1)\left(\frac{F_{E}}{V}\right)} \cdot\left[\left(\frac{R}{R+1}+\left(\frac{F_{E}}{V}\right)\right) x_{i}\right. \\
\left.+\frac{1}{R+1} x_{D}-\left(\frac{F_{E}}{V}\right) x_{E}-y_{i} *\right] \quad i=1, n c
\end{gathered}
$$

For an indirect split with a saturated vapor entrainer $F_{E}$ $(q=0)$, the equation is derived from a bottom envelope mass balance in terms of reboil ratio and bottom flow rate $S$ and bottom flow rate $\mathrm{W}$ :

$$
\begin{aligned}
& \frac{d x_{i}}{d h}=\frac{S+\frac{F_{E}}{W}}{S+1} *\left[\left(\frac{1}{S+\frac{F_{E}}{W}}\right)\left[(1+S) \cdot x_{i}+\left(\frac{F_{E}}{W}\right) \cdot x_{E}-x_{W}\right]\right. \\
& \left.-y_{i} *\right] \quad i=1, n c
\end{aligned}
$$

Eq. (9) is then used to find the relevant equation used in batch operation mode:

$$
\begin{aligned}
\frac{d x_{i}}{d h} & =\left(\frac{S}{S+1}+\frac{F_{E}}{L_{T}}\right) *\left[( \frac { 1 } { ( \frac { S } { S + 1 } + \frac { F _ { E } } { L _ { T } } ) } ) \left(x+\left(\frac{F_{E}}{L_{T}}\right) \cdot x_{E}\right.\right. \\
& \left.\left.-\left(\frac{1}{S+1}\right) \cdot x_{W}\right)-y_{i} *\right] \quad i=1, n c
\end{aligned}
$$

Equations for an indirect split and a boiling liquid entrainer feed can be found in Rodríguez-Donis et al. (2012a) for the batch process and Shen (2012) for the continuous process.

\subsubsection{Rectifying section.}

$\frac{d x_{i}}{d h}=\frac{R+1}{R}\left[\left(\frac{R}{R+1}\right) x_{i}+\frac{1}{R+1} x_{D}-y_{i}^{*}\right] \quad i=1, n c$

In these equations, setting $S$, the reboil ratio, or $R$, the reflux ratio, as infinite and $F_{E}$, the entrainer feed flow rate, equal to zero leads to the residue curve equation (Eq. (1)).

The straightforward calculation method consists in selecting a column configuration and values for the reflux ratio and the entrainer flow rate. Assuming a direct (fixed $x_{D}$ ) or an indirect (fixed $x_{W}$ ) split and a recovery rate, the other product is computed from the overall mass balance as the main feed $x_{F}$ and the entrainer feed $x_{E}$ compositions and flow rates are known. The rectifying liquid composition profile is computed top down from the reflux composition that here, in a homogeneous process, is equal to $x_{D}$. The stripping liquid composition profile is computed bottom up from $x_{W}$. For exploring the extractive composition profiles (the intermediate column section) there are two approaches: (1) the initial point is taken as the pinch point of the rectifying or of the stripping profile or (2) a series of composition profiles can be computed starting from several initial points in the composition triangle. Limiting reflux ratio or entrainer flow rate values can then be found from the map analysis. For a heterogeneous process, see Rodríguez-Donis et al. (2007) for a complete discussion over the seven process variants.

\subsubsection{Batch extractive distillation still path}

Regarding the batch operating mode, additional equations must be considered because of the unsteady state operation that induces changes with time of the composition into the still/boiler $x_{S}$ (direct split, Fig. 1c) or the top vessel $x_{\text {top }}$ (indi- rect split Fig. 1d). The trajectory of the liquid composition in both vessels can be computed as:

For a direct split,

$\frac{d x_{S}}{d t}=\frac{F_{E}}{U_{S}}\left(x_{E}-x_{D}\right)+\frac{D}{U_{S}}\left(x_{S}-x_{D}\right)$

For an indirect split,

$\frac{d x_{T o p}}{d t}=\frac{F_{E}}{U_{T o p}}\left(x_{E}-x_{T o p}\right)+\frac{W}{U_{T o p}}\left(x_{T o p}-x_{W}\right)$

The composition pathway of these vessels is always connected to the instantaneous liquid composition profile in the extractive section. The mathematical form of Eqs. (20) and (21) indicate that the composition pathway is driven by two vectors, defining a cone of evolution, which adds flexibility compared to the continuous process, much like the heterogeneous azeotropic batch distillation process (Rodríguez-Donis et al. 2002).

\subsubsection{Pinch points analysis}

The search for the limiting values of the reflux ratio and entrainer flow rate can be systematized by the use of either an algebraic criterion (Levy et al., 1985; Petlyuk et al., 1999, 2015) or of mathematical approaches like bifurcation theory (Knapp and Doherty, 1994), interval arithmetic (Frits et al., 2006) or the combined bifurcation - short cut rectification body method (Brüggemann and Marquardt, 2004). Except for Petlyuk's work, only the (1.0-1a)-m1 extractive separation class was considered for these works.

Extending its method for single feed azeotropic distillations (Levy et al., 1985), Levy and Doherty (1986) proposed an algebraic trial-and-error tangent pinch points procedure for determining the minimum reflux ratio without the necessity of lengthy iteration schemes involving column profile calculations. The method consisted in finding the value of reflux ratio which makes the feed pinch point, the saddle pinch point, and the controlling feed composition collinear but was restricted to ternary mixtures.

After studying the sequence of the extractive column with the entrainer regeneration column for the separation of the acetone-methanol azeotrope with water (Knapp and Doherty, 1990), Knapp and Doherty (1994) used bifurcation theory to relate the extractive process feasibility to the appearance of saddle-node bifurcation points and branching points. Feasible processes required that a ternary saddle originating from a pure component exists whereas the appearance of a ternary unstable node on the pinch branch originating at the azeotrope led to an unfeasible separation. Studying several mixtures, they proposed the heuristics cited in the previous section to set the operational values of $R$ and $F_{E}$, once their minimal value was known.

Frits et al. (2006) used an interval arithmetic-based branchand-bound optimizer to find limiting flows based on the existence and location of singular points and separatrices in profile maps. Their study in batch operation agreed with that in continuous; the process is feasible under infinite reflux ratio above a minimal entrainer flow rate which corresponds to the merging of a stable pinch point originating from the azeotrope with a saddle point originating from a pure component. Finite reflux ratio analysis showed that the pinch points moved inside the composition triangle and brought unfeasible regions which are described later. 


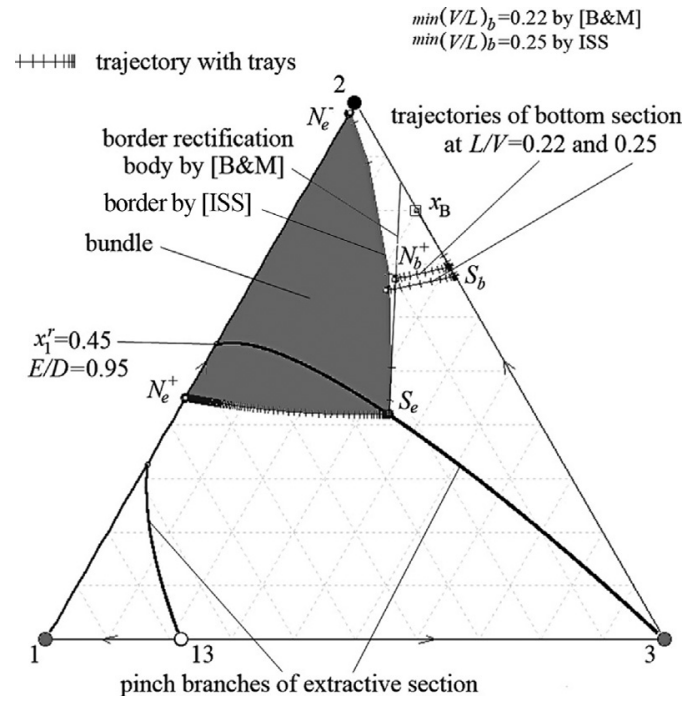

Fig. 13 - Comparison of calculated results for $\min (\mathrm{V} / \mathrm{L}) \mathrm{b}$ obtained by the RBM method B\&M (Brüggemann and Marquardt, 2004) and the ISS method (Petlyuk et al., 2015) (From Petlyuk et al., 2015 with permission from Elsevier).

Brüggemann and Marquardt (2004) exploited a fullyautomated shortcut design procedure to determine the limiting values of the reflux ratio and entrainer flow rate. The method is based on the approximation of all column profiles by the so-called rectification body method (RBM) which is constructed from nonlinear analysis of the pinches of each section (Bausa et al., 1998). Like Knapp and Doherty (1994), they also set some operational constraints to determine the quasi-optimal values once the minimal values of $R$ and $F_{E}$ are known (see Section 3.3.2). Kossack et al. (2008) then used the RBM method as a second screening criterion for evaluating the extractive distillation entrainer candidates. Fast and efficient, the method bears some critics when the profiles are highly curved because each rectification body has straight boundaries (Lucia et al., 2008).

Petlyuk et al. (2015) extended the method of infinitely sharp splits based on pinch analysis to determine the feasibility of extractive distillation at infinite number of trays and finite reflux ratios. They considered four different topologies, belonging to the three classes 1.0-1a, 1.0-2 and 2.0-2b, which differ in the number of azeotropes and the relation between the $\mathrm{K}$ values of the components along the edge of the concentration triangle. The limiting values of entrainer flow rate and reflux ratios can be calculated from the knowledge of the location of pinch points and pinch branches, and the $\mathrm{K}$ values along the edges of the concentration triangle. The authors compared the method with the RBM method of Brüggemann and Marquardt (2004) and noted that the curvature of the bounding trajectories is taken into account in their method, hence a more accurate value of the limiting $\mathrm{V} / \mathrm{L}$ ratio (related to the reflux ratio) can be achieved (See Fig. 13). If the product specifications are given, the number of trays of the column sections can also be determined (Petlyuk et al., 2015).

\subsection{Process control and optimization}

\subsubsection{Control}

3.4.1.1. Control of continuous extractive distillation. Fruit of years of expertise in control, Luyben and Chien (2010) present in their book an overall control strategy for the continuous extractive distillation (CED) that has been used very often in the literature works. The separation of the minimum homoazeotropic mixture isopropanol-water by using DMSO as a heavy entrainer $\left(T_{B P}=190^{\circ} \mathrm{C}\right)$ is studied (Fig. 14).

They determine the inventory control loops (Fig. 14b):

a) The parameters controlled for both columns:

- the level of the reflux drum by modifying the distillate flow rate,

- the top pressure by the condenser duty,

- the reflux ratios by the reflux flow rate (Luyben, 2006).

b) The parameters controlled for the extractive column:

- the bottom level by the bottoms flow rate,

- the entrainer feed temperature by the cooler duty,

- the entrainer flow rate or the entrainer to feed flow rate ratio.

c) The parameter controlled for the entrainer regeneration column:

the bottom level by the entrainer makeup flow rate (Grassi, 1992; Luyben, 2006).

Only tray temperature control loops are applied to keep the product purity in both columns. As usual in distillation, the temperature control points are selected at stages with high sensitivity and nearly linear behaviour. Closed loop dynamic simulations of the response to perturbations on the reboiler duty, on the feed flow rate and composition have been done by these authors and others and showed the adequacy of the strategy. Nevertheless, the problem of this overall control strategy is that the bottom level of the entrainer regeneration column decreases until being almost empty. Another problem is that the valve of the fresh entrainer feed is wide open and the entrainer feed to the extractive column cannot be at its set point.

Hence, alternative strategies have been imagined. Grassi (1992) suggested a modified overall control strategy, in which both reflux flow rates are proportionated to the fresh feed flow rate (Fig. 14b). The same temperature control points are applied for both columns as in Luyben's strategy. Tested with disturbances of $\pm 20 \%$ of the feed composition and feed flow rate, the product compositions are much closer to their specifications than by using the control strategy, in which the reflux ratios are fixed. The bottom level of the entrainer regeneration column is maintained without problem.

Recently, Wang et al. (2016) applied effective relative gain array (ERGA) to find the best loop pairing, and a new strategy was proposed for the dehydration of an ethyl formate-ethanol mixture with ethylene glycol (Fig. 15a). Unlike Luyben's control strategy that indirectly controls the product purity by adjusting the temperature of the sensitive tray, the direct composition control is adopted in the ERGA control strategy to control the product purity by manipulating the reboiler duty. The ERGA strategy performed better than Luyben's strategy for disturbances up to $\pm 50 \%$ of the feed composition and up to $\pm 10 \%$ feed flow rate, especially regarding both column base levels.

Wang et al. (2015c) proposed an improvement on the control structure of Luyben. where the entrainer-to-feed ratio set point of the entrainer flow rate controller was given by a composition controller in a cascade arrangement. The composition controller was applied to keep the composition of the distillate of the extractive column constant, Moreover, the reflux-to-feed ratio (L2/B1) of the entrainer recovery column was controlled instead of keeping the reflux ration constant. Unlike the conventional structure of Luyben, the improved one was able to handle $\pm 20 \%$ disturbances in feed flow rate 
a)

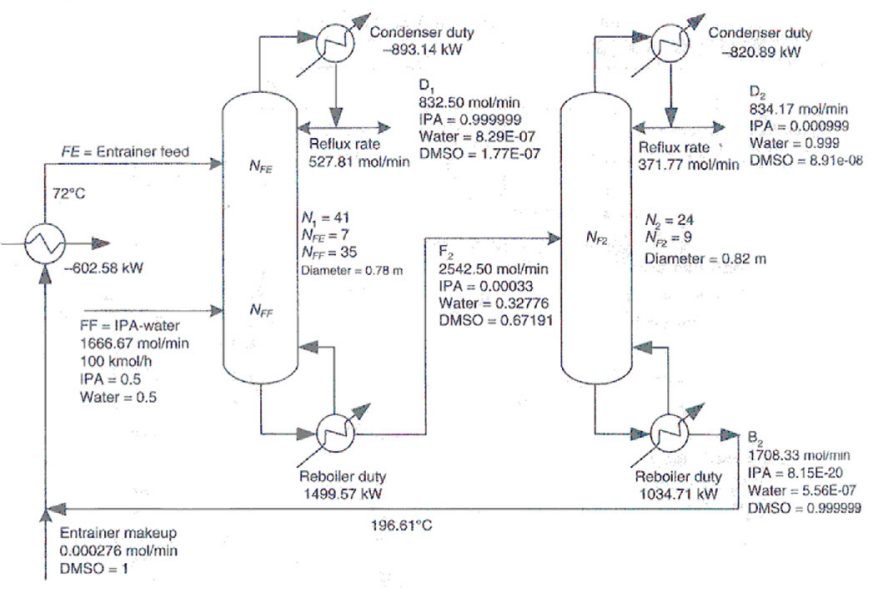

b)

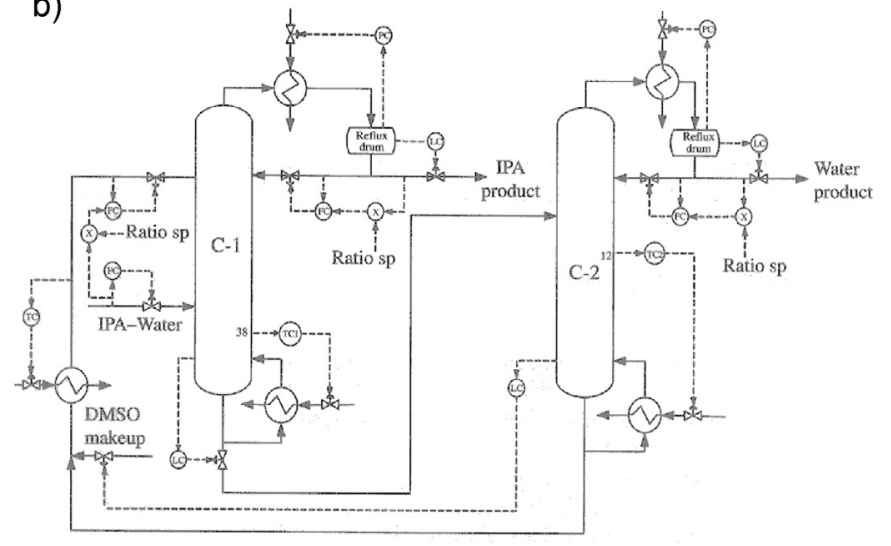

Fig. 14 - Example of optimal design flowsheet (a) and Luyben's control strategy (b) with reflux fixed for a 1.0-1a extractive separation class (Luyben and Chien, 2010, Copyright 2010 Wiley, inspired by Grassi, 1992).

a)

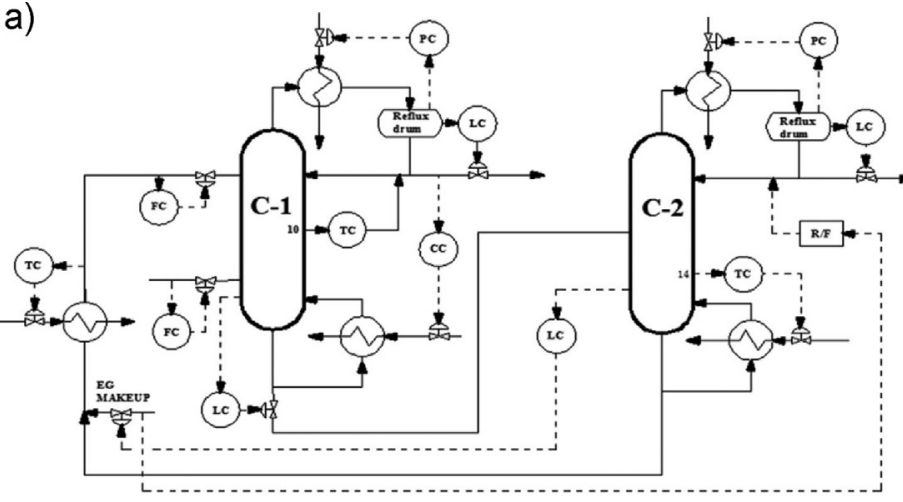

b)

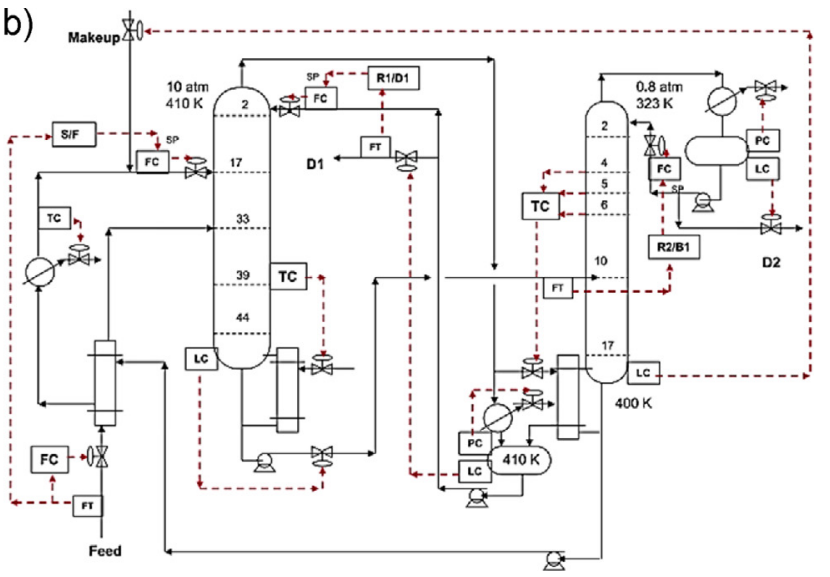

Fig. 15 - Wang's ERGA (a) and Luyben's new control strategy (b) for a 1.0-1a extractive separation class (Wang et al., 2016 Copyright 2016 Wiley; Luyben 2018a, Copyright 2018 Elsevier).

and composition in the separation of ethanol and THF with ethylene glycol as entrainer.

Wang et al. (2015b) showed that an economically optimal design might come at a cost of poor controllability. For the separation of $i$-butanol and $n$-heptane with $N$-methyl2-pyrrolidone as entrainer, various control structures were studied that differed in the way of controlling the reflux flow rate of the extractive column (constant $\mathrm{R}$, constant $\mathrm{L} / \mathrm{F}, \mathrm{R}-$ temperature and L/F-temperature cascades). However, none of these structures gave a satisfactory control performance, and direct composition control was applied, whose action, on the other hand, was very slow. A faster and simpler solution was obtained by applying the basic, constant $\mathrm{R}$ control structure but with increased (by 30\%) entrainer flow rate. The improved control performance came at a cost of $7.7 \%$ increase in TAC. Wang et al. (2018a) also found that in a case study of separating the mixture methanol-water-toluene with $\mathrm{N}$ methyl-2-pyrrolidone as entrainer using two columns and a decanter that increasing the entrainer flow rate from its optimal value was necessary to ensure good controllability.

Cao et al. (2017) proposed another improvement of Luyben's control structure, which did not give a satisfactory performance. Composition control was applied for the extractive column (as by Wang et al., 2015b), while the entrainer recovery column had a L2/B1-temperature cascade controller to manipulate the reflux flow rate.
Recently Luyben (2018a) added an economizer to reduce the reboiler duty in the extractive column. It modifies the feed vapour content, but Luyben noticed that it also induced perturbations especially when higher pressures were used to reduce the entrainer-feed ratio for the extractive distillation process for recovering methanol from acetone with chlorobenzene studied by Luyben (2008b) and You et al. (2017a). The alternative control structure (Fig. 15b) introduced a single change from the conventional Grassi structure (F/ $F_{E}, R 1$ and $\left.R 2\right)$ by changing the reflux to distillate (reflux ratio) scheme to a reflux-to-feed in the entrainer regeneration column $\mathrm{C} 2$. The feed to $\mathrm{C} 2$ is the bottoms B1 from the extractive column, so a R2/B1 ratio is used. It was more performant in coping with feed composition disturbances.

Unlike the strategies above, Gil et al. (2012) proposed another strategy for ethanol dehydration with glycerol that did not use the entrainer make-up flow rate to control the base level in the recovery column. Instead, both columns' base levels are controlled by manipulating the bottom flow rates. Besides, the entrainer flow rate is proportionated to the azeotropic feed and the ratio is controlled by manipulating the make-up flowrate (Fig. 16). Again, the entrainer flow rate control is on cascade with the feed flow rate. Tested with small disturbances $\pm 2 \%$ of the feed composition and up to $\pm 20 \%$ of feed flow rate, this alternative strategy led to smaller perturbations in the temperature response in both columns and 


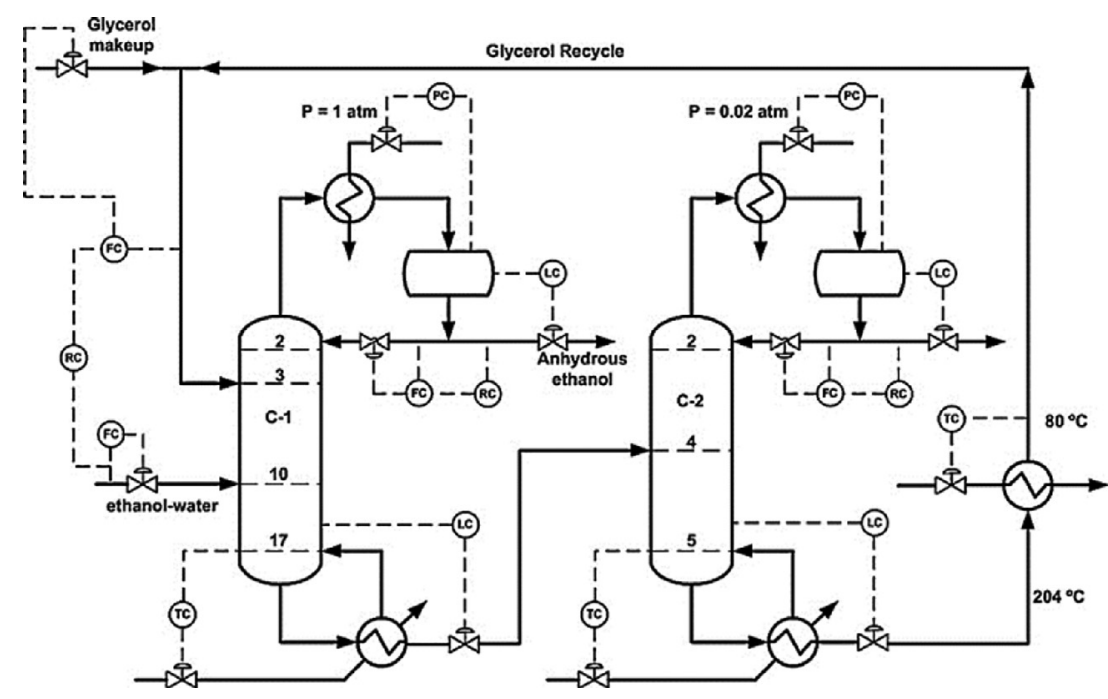

Fig. 16 - Gil's control strategy with reflux fixed for a 1.0-1a extractive separation class (Gil et al., 2012, Copyright 2012 Elsevier).

because of faster dynamics, avoided to increase the value of the sump level of the recovery column to achieve good controllability.

When dealing with heat integrated extractive distillation process, several degrees of freedom are lost and alternative control schemes must be devised. For example, this can arise when combining a preconcentration column with the extractive distillation column or with the entrainer regeneration column (Liang et al., 2014; An et al., 2015; Zheng et al., 2017), or when separating ternary mixtures with non-conventional process with rectifier (Luyben, 2016c).

3.4.1.2. Control of batch extractive distillation. Luyben and Chien (2010) studied the control of the batch extractive distillation (BED) in their book via two examples. The BED process proceeds according to the following steps for an A-B separation with E:

1. Start-up: $R_{1}=\infty$, initially without $E$ and then with $E$ feeding,

2. Production of $A: R_{2}=$ constant, distillate tank: $P 1$ (Fig. 17),

3. Slop-cut collection: $\mathrm{R}_{3}=$ constant, distillate tank: $\mathrm{S} 1$,

4. Production of $\mathrm{B}: \mathrm{R}_{4}=$ constant, distillate tank: $\mathrm{P} 2$,

5. Slop-cut collection (optional),

6. Production of $\mathrm{E}$ at the column bottoms (optional. The prescribed purity of the water often can be reached during Step $4)$.

The reflux is kept constant for the acetone (A)-methanol (B) separation with water $(E)$, and is varied in the distillate step for the isopropanol (A)-water (B) separation with DMSO (E).

There are four control loops (Fig. 17a) for ensuring the stable operation when the reflux and entrainer flow rates are kept constant:

- a ratio control loop setting the reflux ratio by modifying the distillate flow rate,

- a level control loop for the reflux drum,

- a pressure control loop to maintain the column pressure,

- a flow loop for the continuous feeding of the entrainer.

Variation of the reflux ratio and the entrainer flow rate aims at reducing the entrainer consumption. The reflux ratio is varied by a temperature control loop (Fig. 17b).
For the determination of the optimum values of the operational parameters, namely quantity pre-loaded of the entrainer, entrainer flow rate, reflux ratios for each operational step; a pressure-driven simulation was performed in Aspen Dynamics (Luyben and Chien, 2010) with the scope of maximizing the solvent efficiency (amount of product A vs the amount of E used) and the capacity factor (amount of B vs total time) (Luyben, 1988).

3.4.1.3. Optimal control. Optimal control aims at finding the most suitable operational policy through the process operation. The non-linear dynamic optimisation problem that needs to be solved is very challenging (computationally speaking) and thus simplified models as well as efficient solving strategies are required.

Ramos et al. $(2013,2014)$ studied the optimal control profiles of the extractive dehydration of ethanol using glycerol as entrainer when the azeotropic feed was subject to step-wise and sinusoidal disturbances. The goal was to help tailoring nonlinear model-predictive control strategy implemented in a real production facility. They used an index-1 differentialalgebraic equation system and discretized it so as to solve it as non-linear programming problem, subject to constraint on product purity and maximum column temperature. The manipulated variables were the reboiler duty and the molar reflux ratio of condenser.

Mujtaba (1999) analysed the optimal control solution for an extractive batch distillation process. He used the distillate flowrate and the ratio of the entrainer flow rate and main feed flow rate as manipulated variables.

\subsubsection{Optimization}

The problem to be solved is essentially a mixed integer nonlinear programming problem and different objective and constraints can be set. Although the optimization issue is usually solved by using computing, optimal results must be analyzed with respect to other issues like thermodynamics feasibility, thermodynamics efficiency or controllability.

3.4.2.1. Problem setting. The optimization of extractive distillation process is usually focused on the quality of separation (product purity and recovery yields) and on the process energy 
a)

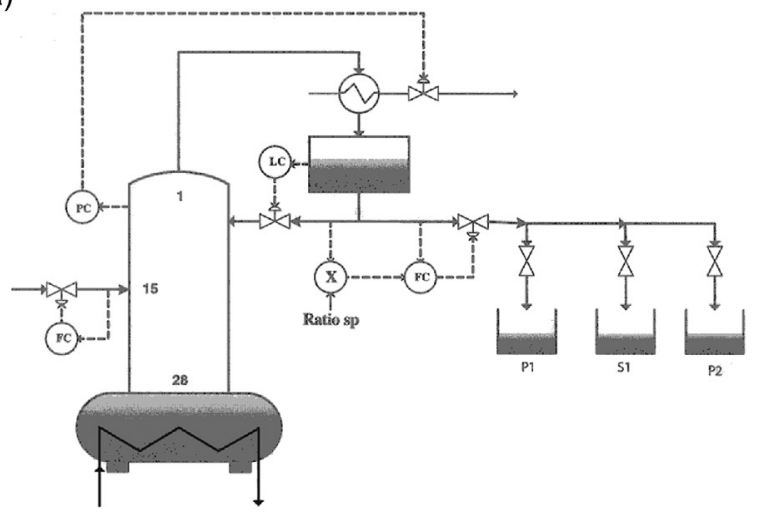

b)

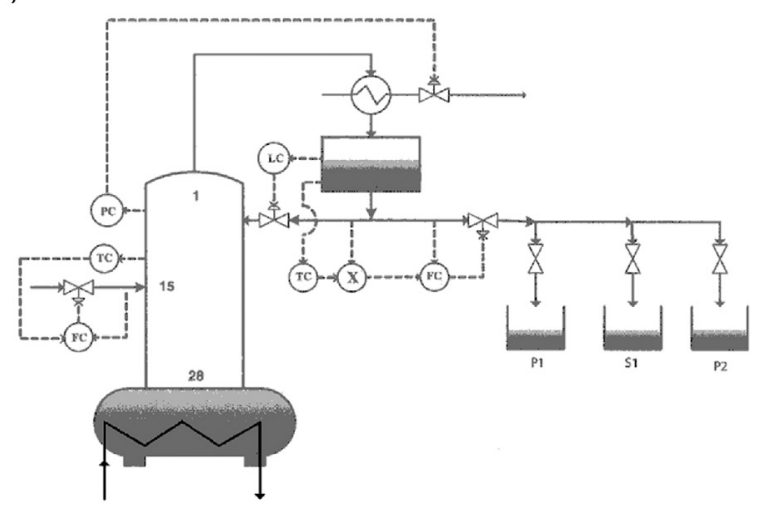

Fig. 17 - Control of batch extractive distillation (a) constant reflux ratio in each operational step; (b) variable reflux and entrainer flow rate (Yao et al., 2007. Copyright 2007 elsevier).

consumption and total annualized costs (TAC). A variety of objective functions has been used in the literature. Minimizing TAC that incorporates both operational and capital costs, is a wide-spread choice (Kossack et al., 2008; Emhamed et al., 2008; Wang et al., 2012, 2015a; Medina-Herrera et al., 2014; Brito et al., 2016; Lo and Chien, 2017; Ahmadian Behrooz, 2017). A similar measure, called economic potential was used by Langston et al. (2005). If capital costs are not taken into account, for example, if the equipment already exists, the energy consumption per overall product flow rate can be minimized (e.g. De Figueirêdo et al., 2011; You et al., 2015a,b) or the profit maximized (Barreto et al., 2011b). The purity specifications are usually set as constraints, either explicitly in the optimization method, or incorporated into the model (Barreto et al., 2011b). Recovery, on the other hand, is usually prescribed by fixing the flow rate of the product of specified purity. However, in some cases, recovery was also used as an optimization constraint (Bravo-Bravo et al., 2010; Kiss and Ignat, 2012). The difference between treating purities or recoveries explicitly as constraints instead of specifying them in the model is that in the former case they are inequality constraints, in the latter they are, as all the model equations, equality constraints. For the optimization of an extractive dividing-wall column, Kiss and Ignat (2012) used two objective functions, the heat duty in an inner loop and the product $N(R+1)$ in an outer loop as its minimum is a good approximation of the minimum of TAC (Dejanović et al., 2011). Wang et al. (2012) applied a similar method, using the sum of the heat duties as the objective function in the inner loop, and TAC in the outer loops.

Besides an economic objective function, some authors also take other aspects into account by using multi-objective optimization. This can be the environmental impact (Barreto et al., 2011a), or the safety of the process (Medina-Herrera et al., 2014). Barreto et al. (2011a) calculated the environmental impact from the amount of $\mathrm{CO}_{2}$ emitted and from the influence of chemicals on human health evaluated by their median lethal dose (LD50) and threshold limiting values (TLV). Medina-Herrera et al. (2014) considered the safety of the process by using the distance likely to cause death as an objective function. Bravo-Bravo et al. (2010) also applied multi-objective optimization to an extractive distillation process including a dividing wall column, implicitly minimizing the cost by minimizing the total heat duty, the number of trays in both the main column and post fractionator and the flow rate of the entrainer.
Some authors focus on the extractive distillation column alone (Hilal et al., 2001, 2002; Gil et al., 2009; Langston et al., 2005; De Figueirêdo et al., 2011), but various authors demonstrated that the entrainer regeneration should be systematically included (Luyben, 2008b; Kossack et al., 2008; You et al., 2015a,b; Skiborowski et al., 2015). This happens in a regeneration column in the continuous process or in the last operating step in the batch process.

Apart from the classical extractive distillation flow sheet, intensified extractive distillation technologies were also optimized. The optimization of extractive dividing-wall columns was considered by Bravo-Bravo et al. (2010), Kiss and Ignat (2012) and Modla (2013). You et al. (2016b) studied a heat integrated extractive distillation system, where the reboiler of the extractive column and the condenser of the regeneration column were coupled. They optimized the system both in the case of full and partial heat integration. For full heat integration, an equality constraint was imposed on the reboiler and condenser heat duties.

Depending on the variables considered, extractive distillation optimization problems are of various kinds: NLP when continuous variables (reflux, entrainer flow rate, pressure, heat duty...) are considered only; MINLP when integer variables are added (total numbers of trays, main feed and entrainer feed locations for both the extractive and the regeneration column). However, one can also optimize discrete decisions, like the screening of entrainer or the selection of utilities. That introduces disjunctions resulting in a mathematical formulation in the form of a generalized disjunctive programming (GDP) problem. That problem was addressed by Skiborowski et al. (2015) who optimized the choice of three steam utilities and screened six entrainers for the extractive separation of the acetone-methanol azeotrope, while optimizing all other process variables as well. Three of the entrainers enable obtaining acetone as first distillate product (1.0-1a-m1 extractive separation class) and three others allow to recover methanol as first distillate product (1.0-1a-m2 extractive separation class).

In the case of batch extractive distillation, as the process is time-dependent, the optimization problem becomes a dynamic one. In a general formulation, the time-optimal profiles of the optimization variables must be determined to obtain the optimal value of the objective function for the complete batch process. Such a problem is of infinite dimensions, and it can be solved by discretization: the process is divided into a limited number of steps in which the optimization 
variables have different but constant values. Therefore, the optimal continuous profiles are estimated by stepwise constant ones. In the simplest case, there is only one batch task, that is, the optimization variables are constant during the operation. If the process has multiple operation steps (tasks), for example if the entrainer regeneration is also studied, or if off-cuts are taken, the optimization variables of the different tasks are treated independently.

Compared to continuous distillation, the existence of an operation time gives rise to new possible objective functions. Mujtaba (2004) referred to optimal control and distinguished several batch distillation optimization problem formulations according to the objective function: minimum time, maximum distillate and maximum profit (or productivity) problems. This classification is applicable to batch extractive distillation, as well. Beyond purity specification, the amount of distillate is an inequality constraint for minimum time problems, and the operation time is an equality constraint for maximum distillate problems. Mujtaba (2004) defined the objective function of the maximum profit problem as:

$P=\frac{\text { added value to product }}{t}-$ operating costs

where $t$ is operation time. This definition was used by Low and Sorensen (2002), for example. On the other hand, it is also possible to use the product $\mathrm{P} \times \mathrm{t}$ as objective function (Hegely and Lang, 2016), but the location of the optimum will be different. Mujtaba (1999) solved an optimal control problem and decomposed a multi-period maximum profit problem into a series of independent single-period problems, which were minimum time or maximum productivity problems, depending on the operation step (task).

3.4.2.2. Solving strategies. Such optimization problems can be solved for the continuous process with different methods. Method selection is also influenced by the modelling approach used. If the model equations are explicitly known such as in the case of using MATLAB, GAMS or other equation-based modelling environment, the optimization problem is usually a mixed-integer nonlinear programming (MINLP) one. Either the optimization algorithm (solver) then can be chosen from the ones already available in modelling environment, or it can be coded by the researchers themselves. Emhamed et al. (2008) used the modified outer approximation of Farkas et al. (2008) implemented in AIMMS to optimize extractive distillation processes. Before optimization, the feasible region was explored by using a factorial experiment. Kraemer et al. (2007) proposed an optimization method called successive relaxed MINLP (SR-MINLP), where the MINLP problem is solved as a succession of nonlinear programming (NLP) problems. The method was implemented in GAMS and tested on the case of study separating ethanol and water using glycol as entrainer. The NLP subproblems were solved using the SNOPT solver. The extractive distillation optimization problem was also solved using the branch \& bound, outer approximation and continuous reformulation MINLP solving methods. SR-MINLP method found the best solution. The method was also used by Kossack et al. (2008) to optimize the separation of acetone and methanol using different entrainers. Garcia-Herreros et al. (2011) used a two-level strategy that combines stochastic and deterministic algorithms. Skiborowski et al. (2015) presented a hybrid evolutionary-deterministic approach for conceptual process design, whose performance was demonstrated on several case studies, including an entrainer-enhanced pressure swing and an extractive distillation process. Their method uses an evolutionary algorithm (EA), where the fitness of each individual is evaluated by local optimization, solving an MINLP problem. Integer variables leading to discontinuities, such as utility and entrainer selection, are handled by the EA. Other discrete variables like numbers of trays and feed locations are handled in the MINLP loop. The hybrid approach reduces the necessary user input for initialization, but some variables ranges are still set. It also generates a variety of approximately equivalent solutions approximating a Pareto front, which can be further evaluated by additional criteria. The EA is implemented in MATLAB, the local optimization algorithm in GAMS.

Another approach is to build the process model in a commercial flow-sheet simulator. Optimization in this case can be performed (1) by a built-in optimization method of the simulator, (2) by following an optimization procedure adapted to the problem, or (3) by coupling the simulation with an external optimizer. The built-in sequential quadratic programming (SQP) optimizer of Aspen is nowadays used routinely in the articles dealing with extractive distillation (De Figueirêdo et al., 2011; Kiss and Suszwalak, 2012; Kiss and Ignat 2013; You et al., 2014, 2015a,b; etc). Wang et al. (2012) proposed a sequential iterative optimization procedure, first optimizing the extractive column in three nested loops, then the entrainer regeneration column in two loops. In each loop, the optimal values of one or more parameters are determined while keeping other parameters constant. If an external optimizer is used, the simulation is treated as a black box model. Therefore, metaheuristic methods, such as genetic algorithm (Leboreiro and Acevedo, 2004; Bravo-Bravo et al., 2010; MedinaHerrera et al., 2014; Modla and Lang, 2012; Modla, 2013; You et al., 2015b) and other evolutionary algorithms are frequently applied, where only the values of the objective function and constraints are required. Sensitivity analysis of the process variables (Gil et al., 2009; Hilal et al., 2002; Langston et al., 2005; Luyben, 2008b) can also be used, independently of the modelling approach.

Caballero et al. (2005) proposed a superstructure-based optimization algorithm that combined the use of a commercial process simulator and generalized disjunctive programming (GDP) with MINLP reformulation. The optimization problem consists of a master MILP problem handling the integer and binary variables, and a NLP problem for the continuous variables with fixed structural configuration. The NLP problem was solved by external solvers, but the use of the process simulator's built-in solvers is also possible. The drawback of the algorithm is that it needs a special tailored master problem that depends on the flow-sheet to be optimized. This drawback was eliminated by Caballero (2015) who proposed a GDP model without MINLP reformulation, eliminating the need of modifying the NLP and MILP subproblems. In this case, the GDP problem is solved by the logic based outer approximation method. These algorithms perform simultaneous optimization of operating conditions and structural parameters, but require good initial values and bounds to converge. Global optimal solutions are not guaranteed. They are not specific to the optimization of extractive distillation, but the applicability to the process was demonstrated through examples both by Caballero et al. (2005) and Caballero (2015).

In most articles, the optimization of the flow sheet is often performed sequentially, that is, the extractive column is optimized first, then the entrainer regeneration column, and built-in SQP optimizer or sensitivity analysis are used for that. However, this is likely to result in a sub-optimal design, 
that one would think sufficient for a grass-root design, but that might miss features that will make the process infeasible. Indeed, the importance of the simultaneous optimization of both extractive and entrainer regeneration columns was noted, as their operational parameters are strongly interdependent (You et al., 2014). As discussed in Section 3.3.1 with Eqs. (10)-(13), the impurity content in the entrainer recycle stream does affect the extractive column distillate flow rate and purity by setting upper feasible limits (You et al., 2016a). Other changes in the process operating conditions (pressures in each column) or in the process structure (adding or not a pre-concentrator; heat integration) motivate the optimization of the process as a whole (You et al., 2018). No work has been devoted to the optimisation of heterogeneous extractive distillation in continuous column.

Optimisation of batch extractive distillation has been less studied, being complicated by the model dynamics. Mujtaba (1999) formulated an optimal control problem to achieve maximum productivity in minimum time under purity constraints, and decomposed the multiperiod problem into independent single-period problems. Lelkes et al. (2003c) used the DICOPT++ solver of GAMS while Frits et al. (2007) used an interval method for that purpose. Hegely and Lang $(2014,2016)$ used a genetic algorithm to optimize the process by taking into account as off-cut or entrainer recycle in addition to usual operating parameters and task durations. For the heterogeneous batch extractive distillation process, Barreto et al. (2011a, 2011b) also used a genetic algorithm to solve the same problem and showed that refluxing a portion of the entrainerlean phase to the column together with the entrainer-rich phase was indeed an optimal solution, as prescribed by the feasibility analysis.

3.4.2.3. Extractive distillation optimisation results. The shape of the temperature profile in zeotropic or azeotropic distillation can be a suitable index for assessing the optimization of the separation process, based on analysis of the second law of thermodynamics that aims at minimizing irreversibilities in distillation separation. Under optimal operating conditions, the T-profile is usually observed to be smoothly increasing from top to bottom. That comes from either the principle of equal thermodynamic distance that leads to keep constant from one tray to the other a quantity proportional to the ratio of the temperature gradient and of the tray temperature (Sauar et al., 2001), or the principle of equipartition of driving force proportional to the chemical potential gradient at vapour-liquid equilibrium over the temperature (Tondeur and Kvaalen, 1987).

In extractive distillation, the feeding of the entrainer, often a high boiling compound, alters the smoothness of the temperature profile and gives rise to irreversible entropy production (Benyounes et al., 2014; Souto et al., 2018), but as evidenced by the numerous literature publishing temperature profiles of optimal design of extractive distillation columns, there should be as minimal disruption as possible (Fig. 18a). Fig. 18 also displays the composition profile for the optimal design in You et al. (2015b). It is noticed that the liquid composition on the entrainer feed tray contains very little methanol (B), in agreement with thermodynamic insight prescribing that composition should match the $\mathrm{SN}_{\text {extr }}$ one, very close to the ternary diagram edge A-E. A sufficient number of trays in the extractive section is recommended for that purpose. Based on our experience and review of literature works in preparation of this manuscript, temperature and composition profiles that bear none of the features highlighted above do not correspond to a so-called optimal design.

The composition profile above reveals the entrainer content on the entrainer feed stage, which corresponds to the $\mathrm{SN}_{\text {ext }}$ node in Fig. 5. For the (1.0-1a) extractive separation class concerned here, we explained that a limit value of entrainers exist. Several authors have investigated concomitantly the effect of that entrainer content on optimal design, either focusing on the $\mathrm{SN}_{\text {extr }}$ location (De Figueirêdo et al., 2015a; Brito, 2015) or analyzing the whole extractive section profile with a separation efficiency indicator (You et al., 2015a,b). They concluded that there exists an optimal range for the entrainer content and for the number of stages in the extractive section, with a compromise between them. High entrainer content requires less trays and less capital cost but impacts energy consumption through vaporisation. Evidently the reflux ratio should be taken into account at the same time to get a more realistic range of feasibility conditions as shown in Fig. 21 later (You et al., 2015b).

Apart from the entrainer selection, and whatever the solving methods or the continuous or batch operating mode, the following variables should be optimized for extractive distillation. These are the numbers of trays of columns, feed locations, reflux ratios (boilup ratio), distillate flow rates (bottom flow rates) and entrainer flow rate (Mujtaba, 1999; Milani, 1999; Munoz et al., 2006; Arifin and Chien 2008; Kossack et al., 2008; Lek-Utaiwan et al., 2011; García-Herreros et al., 2011; Wang et al., 2012; Li and Bai 2012; Duc Long and Lee 2013; Luo et al., 2014; Han et al., 2015; Shen et al., 2015a; Brito et al., 2016; Ebrahimzadeh et al., 2016; Aniya et al., 2016; Zhao et al., 2017b; Li et al., 2017 and others).

Optimization should not only focus on the optimal values of the variables above, but also should refer to useful information such as entrainer feeding temperature, operating pressure and purities of recycled entrainer, prompting for an optimization of all process columns together.

Discarding very high boiling temperature entrainers, on the basis of energy cost for the entrainer regeneration column, is not a universally relevant heuristic as studies have shown that optimization of the ethanol dehydration extractive column with the regeneration column process found similar energy consumption for ethylene glycol $\left(197.3^{\circ} \mathrm{C}\right)$ (De Figueirêdo et al., 2011) and glycerol $\left(287.7^{\circ} \mathrm{C}\right.$ ) (Garcia-Herreros and Gomez, 2011) but a larger consumption for tetraethylene glycol $\left(314^{\circ} \mathrm{C}\right)$ (Ravagnani et al., 2010). Looking at capital and operational costs, glycerol seems more profitable than ethylene glycol: less equilibrium trays in extractive column (18 vs $24)$, a lower entrainer-feed flow rate ratio ( 0.45 vs 0.90$)$ but a larger reflux ratio $(0.35$ vs 0.26$)$. Besides, the mole fraction of ethylene glycol in the bottom product overpasses 0.90 and more demanding conditions for the entrainer regeneration column are expected.

Regarding the common choice of operating the columns at atmospheric pressure, some recent publications have shown that the operating pressure should be optimized as well (You et al., 2015a,b, 2016a, 2017a; Luyben, 2016b; Li et al., 2017; Luyben, 2018a). Care must be taken when lowering the pressure because it might reduce the extractive distillation column temperature in the condenser below what is suitable for using cheap cooling water (You et al., 2015a). But either lowering or increasing should be decided for the benefit of enhancing the volatility. However, no clear trend exists and univolatility curves must be analysed (Zhang et al., 2018a). For several mixtures, such as acetone-methanol with water, 

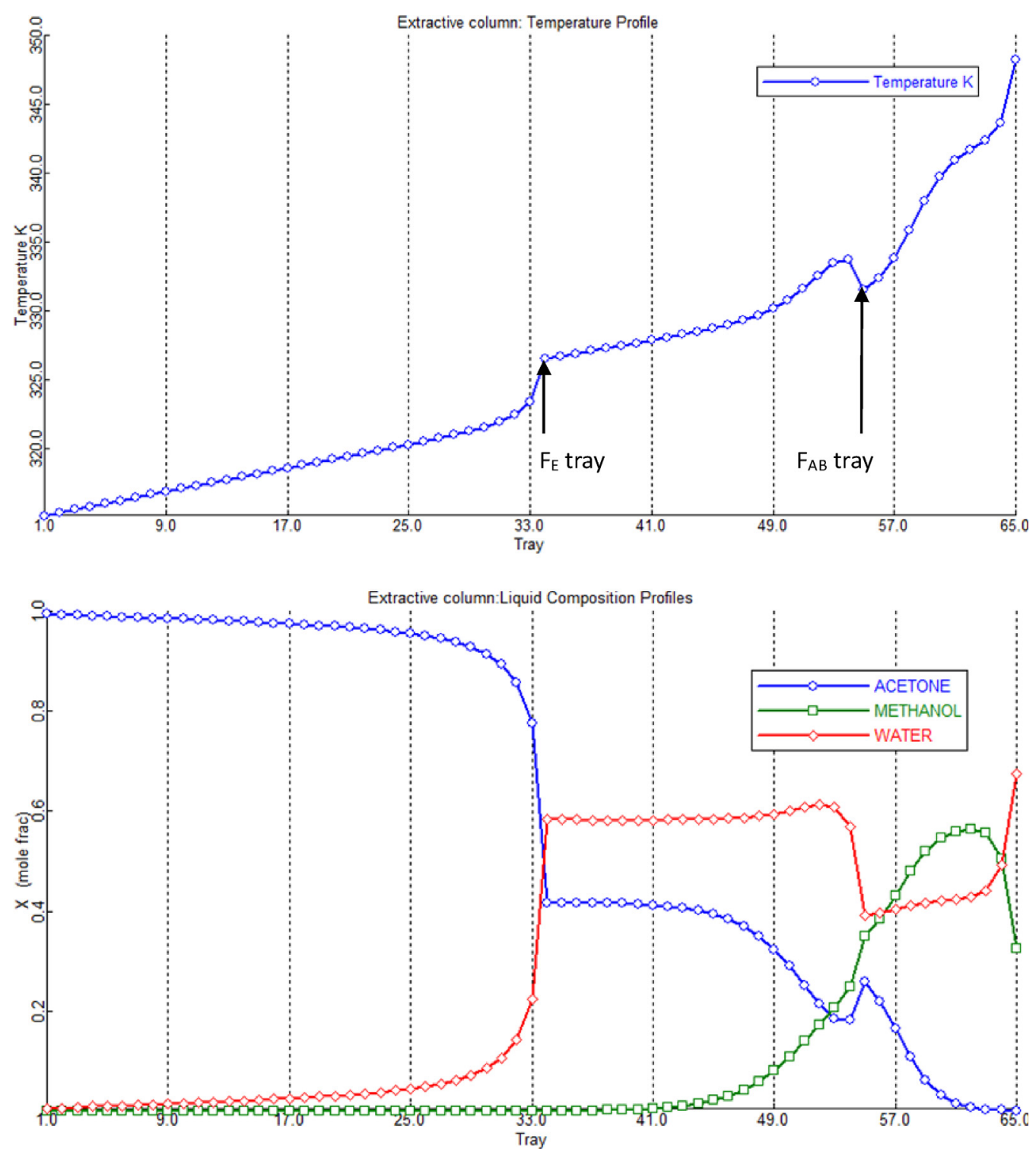

Fig. 18 - Temperature and composition profiles of extractive column for the extractive distillation of acetone-methanol with water, cost optimal design under $\mathrm{P}=0.6 \mathrm{~atm}$ (You et al. 2015b. Copyright 2015 American Chemical society).

the minimal entrainer amount is reduced at low pressure and the $\mathrm{xp}$ point is closer to the desired product vertex as shown in Section 2.4. For 2-methoxyethanol-toluene separation with DMSO, reducing the pressure increases the relative volatility but also increases the minimum entrainer amount (Li et al., 2017). This happens because decreasing pressure enhances more significantly the relative volatility of toluene in the original azeotropic mixture with 2-methoxyethanol than those of each component in their respective binary mixture with DMSO. Hence, the isovolatility lines of toluene shift towards the 2-methoxyethanol vertex where the entrainer concentration vanishes and producing that univolatility line is located farther the toluene vertex. For the recovery of methanol from acetone (A) - methanol (B) with chlorobenzene that sets the univolatility curve $\alpha_{\mathrm{AB}}=1$ to intersect the $\mathrm{B}-\mathrm{E}$ edge and $\mathrm{B}$ to be recovered, it was not decreasing but increasing the operating pressure to $3 \mathrm{~atm}$ that was beneficial: it reduced the entrainer-feed flowrate ratio from 3.52 to 1.90 (You et al., 2017a) and at $10 \mathrm{~atm}$ to 0.72 (Luyben, 2018a).

In addition to operating pressure, several other aspects should be considered simultaneously (You et al., 2018): (1) the necessity of a pre-concentration column (2) the opportunity to avoid the azeotropic composition in the distillate in the pre-concentration column by changing the pressure or other operating parameters. (3) the impact of the impurity content in the recycled entrainer stream as it may limit products recovery yields and purities (see Section 3.3.1) the need to optimize all the sequence columns simultaneously rather than sequentially; because they are coupled and to do so with multi-objective optimizers, because it provides information about suitable parameter ranges and compromise between different objectives. Aspect 2 refers to some common use of feeding the extractive distillation column with $\mathrm{F}_{\mathrm{AB}}$ near its azeotropic composition. There is no justification for that since the azeotrope being a pinch point, it may require many trays and severe reflux conditions to achieve it.

For (1.0-1a)-m1 class, Section 3.3.1 indicates that low contents of impurities in the recycled stream is compulsory to guarantee high purity product. However, for (0.0-1)-H1 class, significant contents of impurities (3\%) in the recycled entrainer was found helpful for reducing the process energy cost (You et al., 2017b). The reason could be explained because of arguments presented in Section 2: for class 1.0-1a, the 

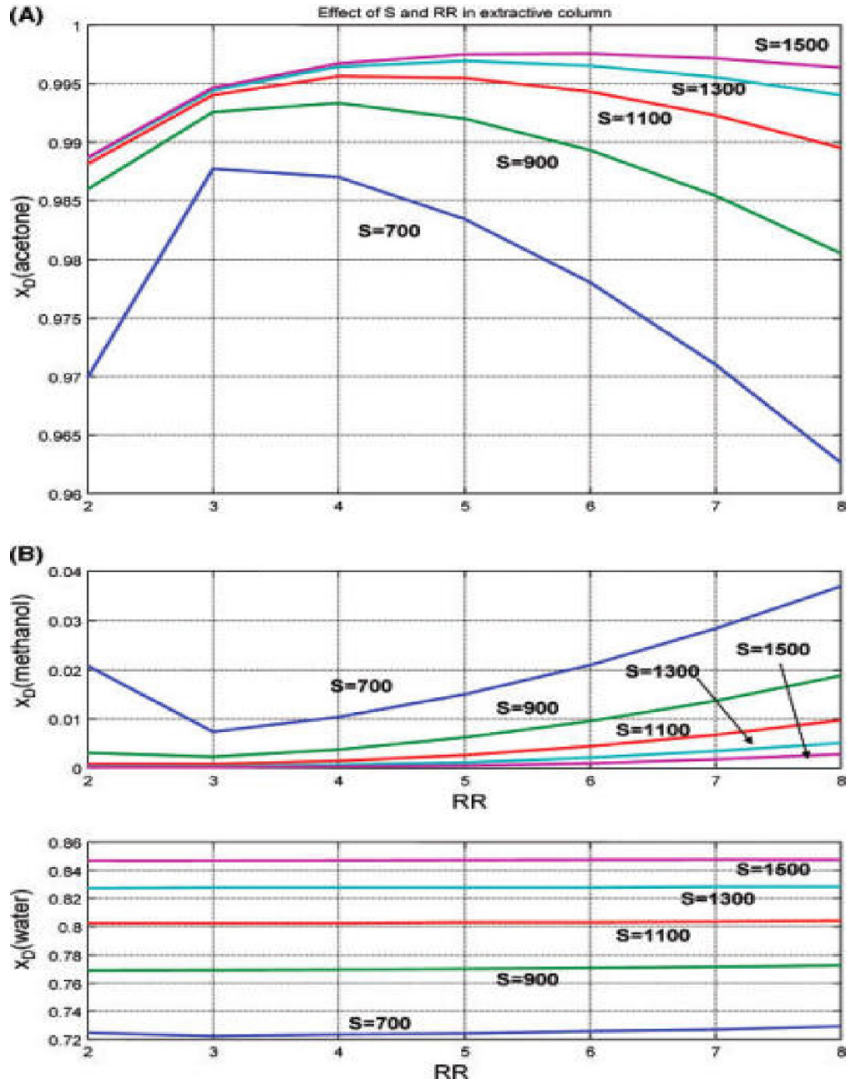

Fig. 19 - Effect of reflux ratio (RR) and entrainer flow rate (S) on product purity (A) and on impurities (B) in the extractive column for the acetone-methanol separation with water (Luyben, 2008a. Copyright 2008 American Chemical Society).

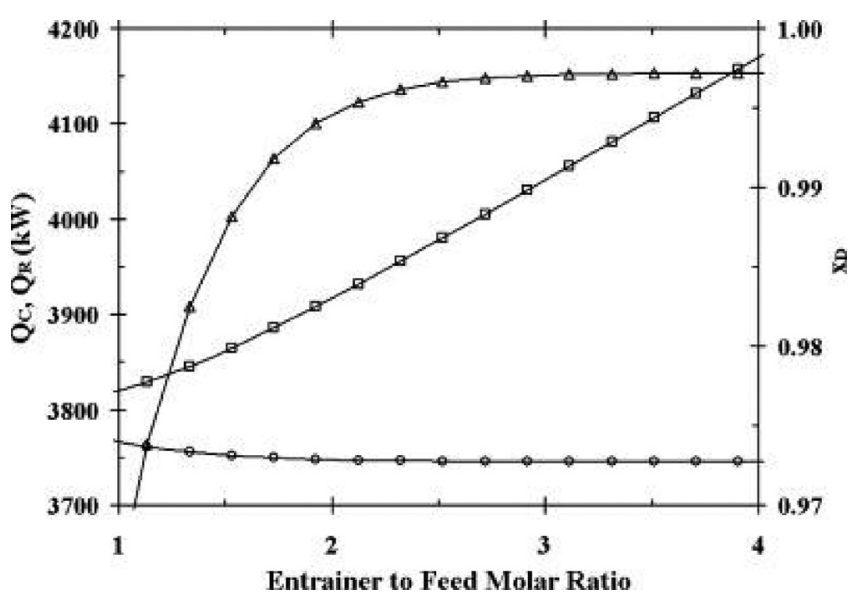

Fig. 20 - Effect of the entrainer to feed molar ratio on distillate composition $(\Delta)$, and heat duties (condenser $O$, and reboiler $\square$ ) (Gil et al., 2009. Copyright 2009 American Chemical Society).

achievable product is a RCM saddle while it is a RCM stable node for class $0.0-1$.

After optimization, some typical results are shown in Figs. 19 and 20, in which the system is acetone-methanolwater.

A sequential optimization shows the effect of the reflux ratio and entrainer flow rate on the distillate purity (Fig. 19) and on the product purity and extractive column energy demand (Fig. 20). A multi-objective optimization displays as a Pareto front some 300 sets of operating parameters enabling

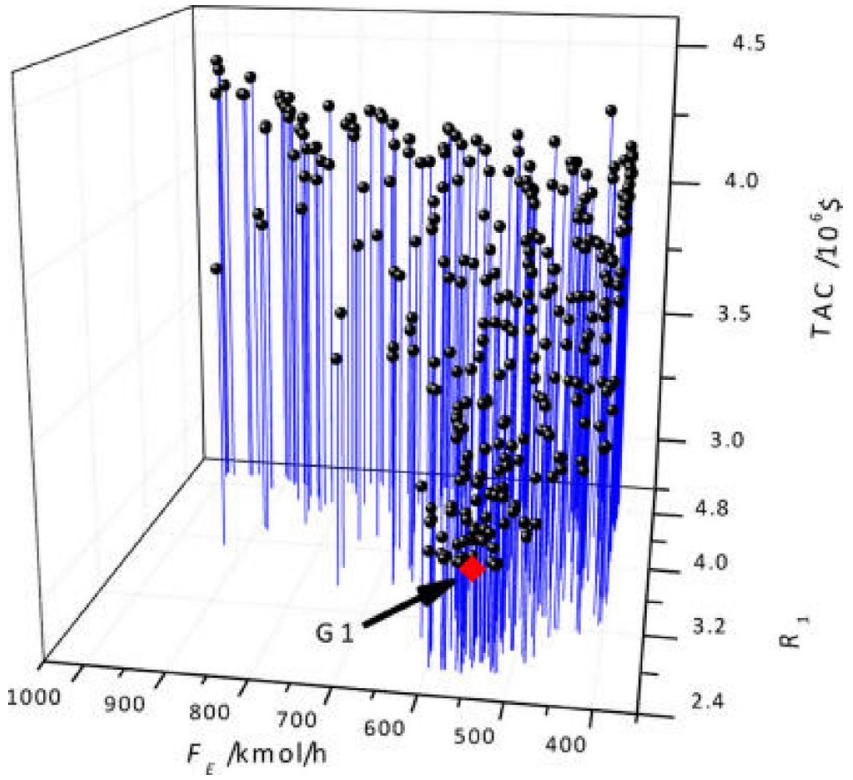

Fig. 21 - Approximate Pareto front of extractive distillation design for acetone-methanol-water system, TAC vs R1 and FE. The red diamond $\mathrm{G1}$ indicates the design with lowest TAC (You et al., 2015b. Copyright 2015 American Chemical Society).

achieving desired purities and yields (Fig. 21). It shows that cost efficient designs are obtained in a narrower range of parameters, hinting at the flexibility of the design.

Fig. 19 shows that on the increase of the entrainer flow rate, the product purity increases while the impurity content decreases. There exists an optimal reflux ratio as well. Above that value, the product purity decreases while impurity content increases at a given entrainer flow rate because for a (1.0-1a) class system the product vertex is a RCM saddle and a high reflux ratio will turn off the rectifying profile to the original azeotropic point away from the saddle pure component. Fig. 20 shows that the entrainer flow rate should not increase too much because the reboiler duty increases quickly. Therefore, the economic value ranges for the entrainer flow rate and reflux ratio seem to converge to each other as shown in Fig. 21. An unsuitable selection of entrainer flow rate or reflux ratio would lead to the rapid increase of TAC.

As exemplified by Fig. 21, several hundreds of design match the purity and recovery constraints. Increasing the number of stages in the extractive section is often correlated with a reduction of the entrainer flow rate and a reduction of the reflux ratio, with a positive impact on the energy consumption (De Figueirêdo et al., 2015b). As shown on Fig. 10 different column profiles correspond to different sets of operating parameter matching the purity. One should look for optimal values in terms of TAC. However it is recommended to relax the optimal design value and some heuristics have been proposed (see Fig. 11 and text). The reason is multiple. According to the discussion in the feasibility section, parameter values close to the limiting values will give rise to profiles closer to the separatrices defining feasible regions. Figs. 11 and 21 show that the feasibility range gets smaller in that case. We have already mentioned in the control section that this kind of stiffness in the design reduces controllability that can be improved by slightly increasing the entrainer flow rate value above its optimal value (Wang et al. 2015b). 


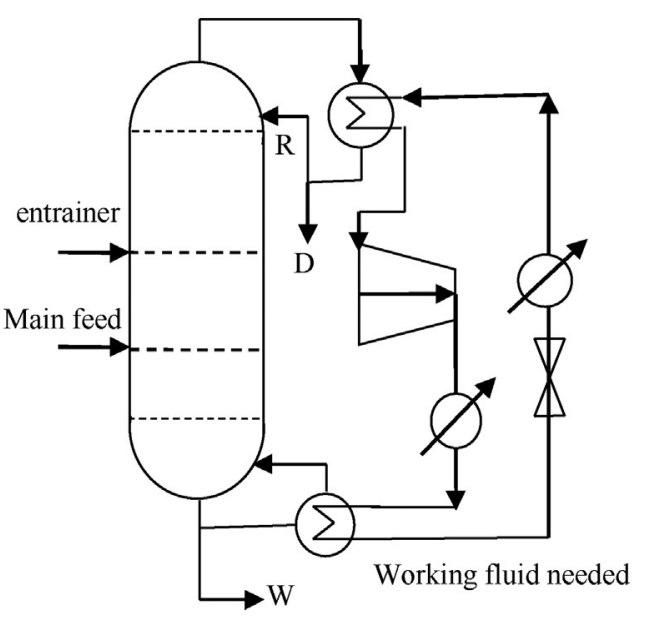

(a) $\mathrm{VC}$

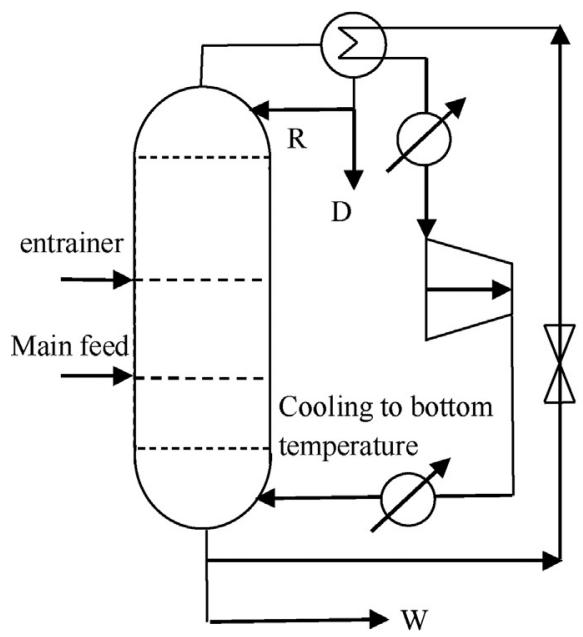

(C) $\mathrm{BF}$

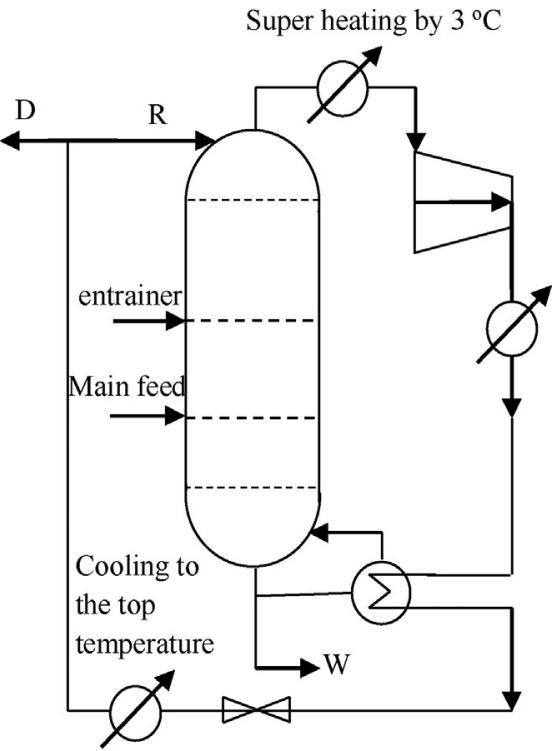

(b) VRC

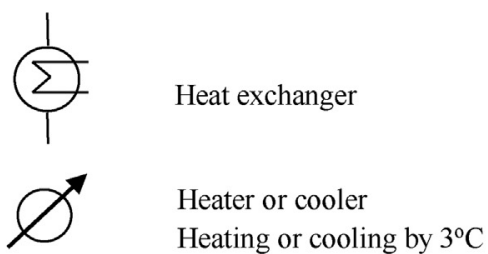

\begin{tabular}{|c|c|}
\hline & Compressor \\
\hline & Throttle valve \\
\hline $\mathrm{D}$ & Distillate \\
\hline W & Bottom liquid \\
\hline $\mathrm{R}$ & Reflux rate \\
\hline
\end{tabular}

Fig. 22 - Mechanical heat pump flow sheet for extractive distillation columns (a) vapour compression (VC), (b) vapour recompression (VRC), (c) bottom flash (BF). (You et al., 2016b. Copyright 2016 Elsevier).

\section{Saving energy for extractive distillation}

In order to further reduce energy consumption of extractive distillation, three main energy-saving methods are considered: heat integrated extractive distillation, extractive dividing wall column and heat pump assisted extractive distillation (Li et al., 2016).

\subsection{Heat integrated extractive distillation}

A possible method to reduce the energy requirements of continuous extractive distillation is to carry out heat integration. We remark that despite promising figures in terms of energy savings, industrial implementation is rare. This can be achieved with the double-effect heat integration of the extractive and the entrainer regeneration column, where the heat duty of one of the reboilers is supplied partially or totally by condensing the top vapour of the other column (Fig. 22) (Lynn and Hanson, 1986). The heat-integrated reboiler is usually that of the extractive column, especially in the case of heavy entrainers, as the lowest temperature difference lies between the bottom of extractive column and the top of the entrainer regenerator column. However, the top of the entrainer regenerator column generally has a lower temperature than the bottom of the extractive column. Either this implies that the pressure of the extractive column has to be decreased or that of the entrainer regeneration column increased to enable heat integration. In this case, it may be called entrainer-enhanced pressure swing distillation, as it can be considered a combination of pressure swing and extractive distillation. Nevertheless, the effect of pressure change on the VLE conditions has to be taken into account, as well. By changing the pressure, azeotropes and isovolatility lines may move, new azeotropes may appear, and existing ones may disappear. Both increasing or decreasing the pressure have 
been found beneficial for the separation (You et al., 2015a; Skiborowski et al., 2015; Luyben 2016b; Li et al., 2017; You et al., 2017a; Zhang et al., 2018a; Luyben, 2018a).

The choice of entrainer will influence the extent of heat integration of the columns, as it governs the temperatures in the columns. Tiverios and van Brunt (2000) applied a heat integration criterion also in their entrainer selection method, deciding whether the condenser of the extractive column (10 bar) and the reboiler of the entrainer regeneration column (atmospheric pressure) could be integrated. If the condenser duty of the extractive column is lower than the reboiler duty of the entrainer regeneration column, the entrainer candidate is rejected.

Full heat integration can be achieved by optimizing the operational parameters of the columns (e.g. reflux ratios), so that the condenser and reboiler duties become equal. However, full heat integration may increase the TAC or have no significant reduction in TAC (Knapp and Doherty 1990; Palacios-Bereche et al., 2015). You et al. (2016a,b) found that there is an optimum amount of heat exchanged with respect to the specific energy consumption, which they determined using a four-step optimization method (You et al., 2014). They also noted that for the separation of acetone and methanol with water as entrainer, the optimal partially heat integrated process is more economical than direct partialy and direct fully heat integrated extractive distillation processes.

The heat integration is qualified as partial when the condenser and reboiler duties are not equal, and additional heating or cooling is necessary. Besides, it should be considered carefully because an unsuitable partial heat integration for extractive distillation may increase rather than decrease the TAC compared with the extractive distillation without heat integration (Luo et al., 2014).

Another approach to reduce energy consumption is the partial thermal coupling of the columns. In some cases, a reduction up to $20-30 \%$ can be reached compared to a conventional extractive distillation flowsheet (Timoshenko, 2004; Gutiérrez-Guerra et al., 2009; Anokhina and Timoshenko, 2015; Timoshenko et al., 2015). Errico and Rong (2012) presented a systematic method for the generation of new distillation sequences and applied it for the case of extractive distillation for bioethanol production. The methodology can produce improved configurations over the basic one. It is understood that the relative advantages of the derived configurations will be different when different entrainers and different feeds are considered for bioethanol productions. Timoshenko et al. (2015) generated a set of extractive distillation flowsheets with partially thermally coupled columns using the graph algorithm synthesis (Timoshenko and Serafimov, 2001; Timoshenko et al., 2001; Timoshenko, 2004) for the separation of ternary mixtures with heavy entrainers. They identified the feasible flow-sheets for all Serafimov types of ternary mixtures, thus reducing the search space for process design. In a case study, the number of potential flowsheets was reduced from 22 to 10 for the separation of the mixture benzene-cyclohexane-toluene with $\mathrm{N}$-methylpyrrolidone as entrainer.

\subsection{Extractive dividing wall column}

Extractive dividing wall column (E-DWC), putting the extractive and regeneration columns into one shell, exhibits the advantages of saving both energy and capital costs. BravoBravo et al. (2010) seems to be the first to consider the extractive dividing wall column (E-DWC). They presented three possible flowsheets for extractive dividing wall column as shown in Fig. 8. Staak and Grützner (2017) published an industrial case study based on a E-DWC developed by Lonza AG. Most literature works simulate E-DWC with equivalent conventional process, but a current challenge remains the development of E-DWC design methods (Cordeiro et al., 2017), Kiss and Ignat (2012) studied E-DWC to concentrate and dehydrate bioethanol in a single step by integrating all units of the conventional sequence, including a pre-concentrator into only one distillation column. The results show that energy savings of $17 \%$, and a similar decrease in capital cost were achieved. Similarly, Xia et al. (2012) studied the E-DWC for separating methylal-methanol mixture and found that energy cost and capital cost are reduced by $8.3 \%$ and $4.1 \%$, respectively. Zhang et al. (2014) investigated E-DWC for ethyl acetate-isopropyl alcohol mixture and showed a $10 \%$ reduction in TAC. Sun et al. (2014) found that a $4.8 \%$ reduction of TAC was achieved by E-DWC for separating benzene-cyclohexane mixture. Li et al. (2017) showed that E-DWC reduced TAC compared to reduced pressure extractive distillation (EDRP) for separating the toluene-2-methoxyethanol mixture thanks to lower capital costs as the EDRP showed lower energy costs. Tavan et al. (2014) showed more than $51 \%$ reduction in energy cost of E-DWC while TAC was not calculated. However, Wu et al. (2013) investigated the energy-saving potential of an E-DWC for four extractive distillation systems and found that only one was suitable. They found that the total reboiler duty cost is reduced but not the actual steam cost, because the boiling temperature of the entrainer is often high and costly high temperature steam grade are required. In addition, the control performance of the E-DWC is hampered because of losing one important control degree-of-freedom. Recently, Patrascu et al. (2017) used heat-pump assisted E-DWC of Luo et al. (2015) to dehydrate ethanol with ethylene glycol, instead of a three column (pre-fractionnator, extractive and regeneration column) and investigated the control issue (Fig. 23a). Using the configuration c in Fig. 8, the liquid split ratio is null as the feed is fed at low temperature at the top of the inner wall and acts as reflux in the left part of the wall. Since the main feed is preheated, feed flow rate disturbance lead to unstable behaviour. With the loss of an internal degree of freedom due to the process intensification, the E-DWC must be stabilized with the help of an additional duty to the side reboiler fed by the vapour recompressed as Fig. 23a shows.

Li et al. (2017) evaluated three control structures for E-DWC, one classical following Luyben's scheme (Luyben and Chien, 2010), another by adjusting $\mathrm{Q}_{\mathrm{B}} / \mathrm{F}_{\mathrm{E}}$ and the vapour split ratio in the column and one by adjusting $\mathrm{Q}_{\mathrm{B}} / \mathrm{F}_{\mathrm{E}}$ and $\mathrm{RR} 1$. The later was more efficient in holding the product purities.

Luyben (2018b) studied the controllability of the E-DWC configuration with a wall all the way up to the top (Fig. 23b) and proposed to manipulate the pressure on both sides so as to manipulate the vapour-split, a necessity justified to maintain the product purity (Tututi-Avila et al., 2014).

\subsection{Heat pump assisted extractive distillation}

Heat pump assisted extractive distillation such as vapour compression (VC), vapour recompression (VRC) and bottom flash (BF) shown in Fig. 22 are useful ways to improve energy quality and reduce the greenhouse emissions in spite of disadvantages like high investment cost and process complexity. 

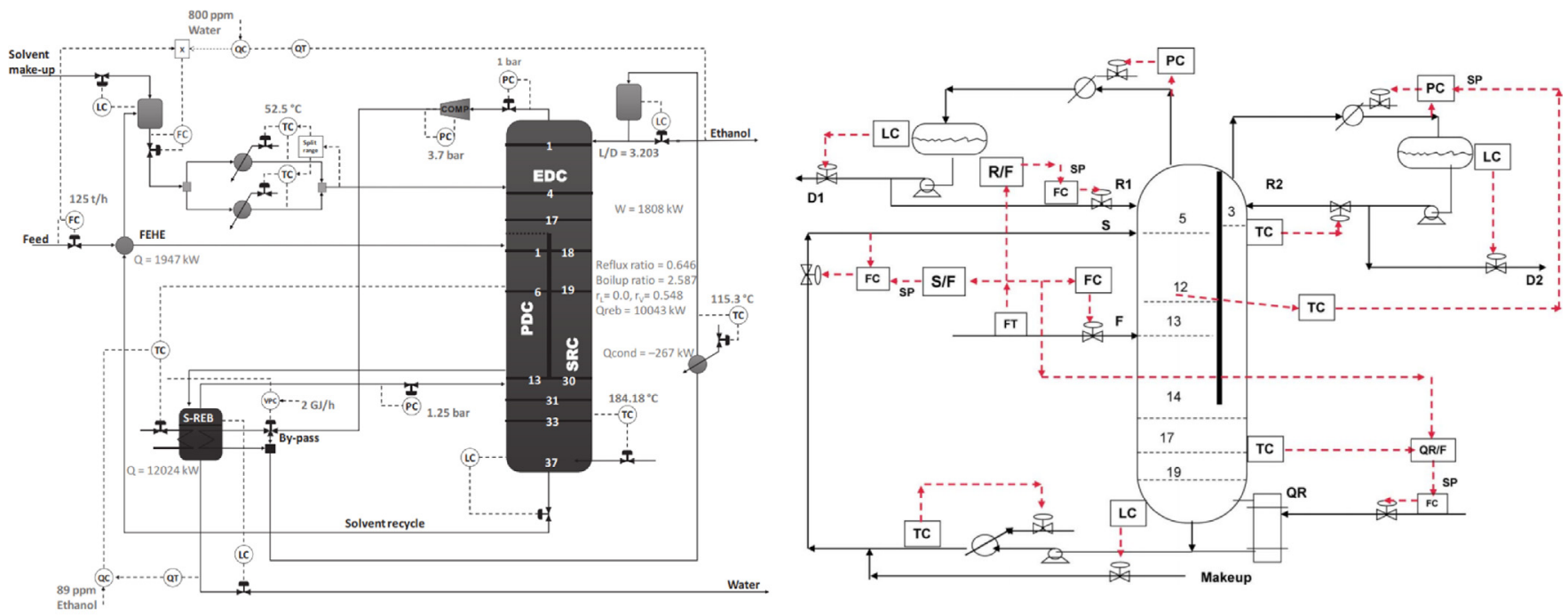

b)

Fig. 23 - (a) New control structure for VRC assisted extractive divided wall column (Patrascu and Bildea, 2017, with permission from Elsevier) and (b) E-DWC with wall all the way to the top (Luyben, 2018b, with permission from Elsevier).

In VC, a working fluid absorbs the heat from condenser and gives it off to the reboiler. In VRC, the top vapour flow is directly compressed and condensed for heating the reboiler. In $\mathrm{BF}$, the bottom liquid is cooled down by a throttle valve and evaporated at the condenser to take off the heat duty (Fonyo and Benkö, 1998; Díez et al., 2009). The advantage of VRC and BF over VC is that a smaller heat transfer area and a lower temperature lift are used because the heat is exchanged only once.

Several studies (Díez et al., 2009; Modla and Lang, 2013; Flegiel et al., 2015) show that VRC process seems to have better performance in economical view over the $\mathrm{VC}$ and $\mathrm{BF}$ processes but this depends on the electricity price and on the temperature lift (Chew et al., 2014; Reddy et al., 2014). However, there is no undisputed thermodynamic reason to prefer VRC process. Useful selection schemes (Kiss and Olujić, 2014) and performance maps (Van de Bor and Ferreira, 2013) for a preliminary choice of different heat pump technologies must be supplemented by comparison of each process simulation. Recently, You et al. (2016b) proposed the partial VRC and partial BF processes for extractive distillation by adding heat pump between the extractive and regeneration columns. They showed that the new processes could increase the coefficient of performance by $60.7 \%$ and $40.0 \%$ compared with the conventional processes because of the small temperature difference between the bottom of the extractive column and the top of the regeneration column. Luo et al. (2015) propose a new VRC assisted E-DWC process ethanol dehydration via using the top vapor stream of E-DWC to drive the side reboiler for water vaporization. The results show that the energy savings of over $40 \%$ and TAC reduction of about $24 \%$ are possible.

\section{Experimental works}

Compared with studies on the measurements of vapour-liquid equilibrium (VLE) and the processes based on VLE data such as process feasibility, synthesis, design, optimization, control and so on, experimental studies on extractive distillation published in the open literature are scarce because conducting a very large number of experiments is neither practical nor economical (Deorukhkar et al., 2016). However, it is necessary to carry out experiments in a pilot plant to investigate the mass transfer efficiency and thus the rate-based model with the aim of predicting the performance of a real extractive distillation process. This is of great importance at high entrainer-to-feed ratios where liquid viscosities increase and can become a limiting factor for mass transfer (Weiss and Arlt, 1987). In addition, the physical and transport properties: density, viscosity and surface tension at various temperatures are needed for predicting the mass transfer efficiency (Quijada-Maldonado et al., 2016).

Kumar et al. (1984) seems among the first authors to publish experimental data of extractive distillation. The author corroborated the experimental data by Murphree efficiencies for the acetone-methanol with water system and the mass transfer models were fitted to process data at various operating conditions. Berg and Yeh (1985) published experimental data for the azeotropic mixtures isopropyl ether-acetone and isopropyl ether-methyl ethyl ketone with DMSO, showing how volatility could be reversed by using extractive distillation (extractive separation class 1.0-1a-m2). Weiss and Arlt (1987) further explained that the decrease in mass transfer efficiency with increasing entrainer concentration was caused by a decrease and partial blocking of the interfacial area caused by the entrainer. They proposed a mathematical model for mass transfer in extractive distillation and applied it successfully to an industrial column. Resa et al. (2000) experimentally studied the extractive distillation for acetone-isopropyl ether with the aim of selecting different entrainers. Lang et al. (2006) ran batch extractive distillation experiments with a new operating policy for separating acetone-methanol-ethanol-water. Xu and Wang (2006) conducted batch extractive distillation experiments and found an effective entrainer 1,2-propanediol for tetrahydrofuran dehydration process. Pacheco-Basulto et al. (2012) carried out batch extractive distillation experiments for the dehydration of bioethanol with ethylene glycol or ionic liquids. Van Kaam et al. (2008) verified their feasibility analysis by experiments for separating chloroform-methanol mixture in batch extractive distillation. Deorukhkar et al. (2016) studied batch and continuous extractive distillation in experiments and found that dimethyl sulfoxide is a more appropriate entrainer than 1,2-propanediol for tetrahydrofuran dehydration. Zhan et al. (2018) carried out experimentally batch extractive distillation for the separation of dimethyl 
disulfide from methyl tert-butyl ether by using diethylene glycol, dimethyl formamide and DMSO that had been screened from quantum mechanical calculations of interaction energies and dissipative particle dynamics of in the entrainers.

Ionic liquids show high effectiveness in separating azeotropic mixtures but their high viscosities could markedly decrease the mass transfer efficiency of the column. Therefore, with the objective of validating a developed rate-based model for the separation of water-ethanol mixtures by using 1-ethyl-3-methylimidazolium dicyanamide and ethylene glycol as entrainers, Quijada-Maldonado et al. (2013, 2016) investigated the effect of the entrainer physical properties on mass transfer efficiency and found that the rate-based model could predict the performance of the pilot plant very well for all the studied conditions. They also observed that the high liquid viscosity decreases the mass transfer efficiency in the rectifying section of the extractive distillation column.

\section{Conclusion}

Overall, the field of extractive distillation has greatly improved over the last twenty years. It can now rely upon a set of complementary approaches, for assessing feasibility on the basis of extractive separation classes that we describe in extenso, for finding relevant operating parameter values, for simulating and optimizing the process and for investigating its controllability.

By extractive distillation azeotropic and low relative volatility mixtures can be separated, and any kind of entrainer can be used, heavy light or intermediate boiling; homogeneous and heterogeneous solvents; classical solvents, ionic liquids or deep eutectic solvents. Unlike azeotropic distillation, the entrainer is fed at another location than the original mixture to be separated, inducing an extractive section within the column.

Extractive separation processes were classified in terms of feasibility relying upon the knowledge of residue curve map topology and univolatility curves along with selectivity ratios. In most cases, the achievable product is a saddle point of the residue curve map, which is an advantage over azeotropic distillation. Depending on the separation class, a direct or an indirect split column configuration allows obtaining a distillate or a bottom product. Batch and continuous processes differ mainly in the feasible ranges for the entrainer-feed flow rate ratio $\left(\mathrm{F}_{\mathrm{E}} / \mathrm{F}_{\mathrm{AB}}\right)$ and reflux ratio $(\mathrm{R})$. Besides, the batch process enables to stir the still path by changing $R$ during the operation. For each type of entrainer, extractive separation classes are defined summarizing feasibility regions, products and $F_{E} / F_{A B}$ limits. Case studies for each separation classes are provided as Supplementary material.

Optimisation of the extractive process should systematically consider the extractive column along with the entrainer regeneration column because the latter requires a lot of energy and may limit the product purity and yield in the extractive column.

Energy and total costs can be reduced by changing pressure or using mixed entrainers. New process structures have also been proposed, namely heat integrated extractive distillation, extractive divided wall column or processes merging a preconcentrator with either the extractive or the entrainer regeneration column. Except the extractive dividing wall column, most of them remain purely theoretical. Several control schemes have been investigated for all process alternatives.
Future work will likely be directed towards new case studies, possibly with uncommon entrainers like ionic liquids or deep eutectic entrainers, that should obey the known feasibility rules; and with the practical implementation of energy savings configurations.

\section{Acknowledgment}

P. Lang and L. Hegely have received financial support from the Hungarian Scientific Research Fund OTKA-NKFI, project No.: $\mathrm{K}-120083$.

\section{Appendix A. Supplementary data}

Supplementary data associated with this article can be found, in the online version, at https://doi.org/10.1016/ j.cherd.2018.09.020.

\section{References}

Acrivos, A., Amundson, N.R., 1955. On the steady state fractionation of multicomponent and complex mixtures in an ideal cascade. 5. The extension to packed columns. Chem. Eng. Sci. 4, 206-208.

Ahmadian Behrooz, H., 2017. Robust design and control of extractive distillation processes under feed disturbances. Ind. Eng. Chem. Res. 56, 4446-4462.

An, Y., Li, W., Li, Y., Huang, S., Ma, J., Shen, C., Xu, C., 2015. Design/optimization of energy-saving extractive distillation process by combining preconcentration column and extractive distillation column. Chem. Eng. Sci. 135, 166-178.

Aniya, V., De, D., Satyavathi, B., 2016. Comprehensive approach toward dehydration of tert-butyl alcohol by extractive distillation: entrainer selection, thermodynamic modeling and process optimization. Ind. Eng. Chem. Res. 55 (25), 6982-6995.

Anokhina, E.A., Timoshenko, A.V., 2015. Criterion of the energy effectiveness of extractive distillation in the partially thermally coupled columns. Chem. Eng. Res. Des. 99, 165-175.

Arifin, S., Chien, I.L., 2008. Design and control of an isopropyl alcohol dehydration process via extractive distillation using dimethyl sulfoxide as an entrainer. Ind. Eng. Chem. Res. 47 (3), 790-803.

Bai, F., Hua, C., Li, J., Xiong, S., 2017. Research progress of deep eutectic solvents in extraction/extraction distillation separation. Xiandai Huagong/Mod. Chem. Ind. 37 (2), 20-24.

Barreto, A.A., Rodriguez-Donis, I., Gerbaud, V., Joulia, X., 2011 a. Multi-objective optimization of three-phase batch extractive distillation, in: Pistikopoulos, E.N., Georgiadis, M.C., Kokossis, A.C. (Eds.). Comput. Aided Chem. Eng., 562-566.

Barreto, A.A., Rodriguez-Donis, I., Gerbaud, V., Joulia, X., 2011 b. Optimization of heterogeneous batch extractive distillation. Ind. Eng. Chem. Res. 50 (9), 5204-5217.

Batista, E., Meirelles, A., 1997. Simulation and thermal integration SRV in extractive distillation column. J. Chem. Eng. Jpn. 3, 45-51.

Bausa, J., Watzdorf, R.V., Marquardt, W., 1998. Shortcut methods for nonideal multicomponent distillation: 1. simple columns. AIChE J. 44 (10), 2181-2198.

Benedict, M., Rubbin, L.C., 1945. Extractive and azeotropic distillation I. theoretical aspects. Trans. Am. Inst. Chem. Eng. 41, 353-370.

Benyahia, K., Benyounes, H., Shen, W.F., 2014. Energy evaluation of ethanol dehydration process with glycols mixtures as entrainer. Chem. Eng. Technol. 37 (6), 987-994.

Benyounes, H., Shen, W., Gerbaud, V., 2014. Entropy flow and energy efficiency analysis of extractive distillation with a heavy entrainer. Ind. Eng. Chem. Res. 53 (12), 4778-4791. 
Berg, L., 1983. Separation of benzene and toluene from close boiling non-aromatics by extractive distillation. AIChE J. 29, 961-966.

Berg, L., Yeh, A.I., 1985. The unusual behavior of extractive distillation. Reversing the volatility of the acetone-isopropyl ether system. AIChE J. 31, 504-506.

Bernot, C., Doherty, M., Malone, M.F., 1990. Patterns of composition change in multicomponent batch distillation. Chem. Eng. Sci. 45, 1207-1221.

Bernot, C., Doherty, M., Malone, M.F., 1991. Feasibility and separation sequencing in multicomponent batch distillation. Chem. Eng. Sci. 46, 1311-1326.

Bravo-Bravo, C., Segovia-Hernandez, J.G., Gutierrez-Antonio, C., Duran, A.L., Bonilla-Petriciolet, A., Briones-Ramirez, A., 2010. Extractive dividing wall column: design and optimization. Ind. Eng. Chem. Res. 49, 3672-3688.

Brito, R.P., 2015. Optimization of the design and operation of extractive distillation processes. Sep. Sci. Technol. (Philadelphia) 50 (14), 2238-2247.

Brito, K.D., Cordeiro, G.M., De Figueirêdo, M.F., Vasconcelos, L.G.S., Brito, R.P., 2016. Economic evaluation of energy saving alternatives in extractive distillation process. Comput. Chem. Eng. 93, 185-196.

Brüggemann, S., Marquardt, W., 2004. Shortcut methods for nonideal multicomponent distillation: 3. extractive distillation columns. AIChE J. 50, 1129-1149.

Caballero, J.A., 2015. Logic hybrid simulation-optimization algorithm for distillation design. Comput. Chem. Eng. 72 284-299.

Caballero, J.A., Milan-Yanez, D., Grossmann, I.E., 2005. Rigorous design of distillation columns: integration of disjunctive programming and process simulators. Ind. Eng. Chem. Res. 44, 6760-6775.

Cao, Y., Hu, J., Jia, H., Bu, B., Zhu, Z., Wang, Y., 2017. Comparison of pressure-swing distillation and extractive distillation with varied-diameter column in economics and dynamic control. J. Process Control 49, 9-25.

Chen, Y.-C., Hung, S.-K., Lee, H.-Y., Chien, I.-L., 2015. Energy-saving designs for separation of a close-boiling 1,2-propanediol and ethylene glycol mixture. Ind. Eng. Chem. Res. 54 (15), 3828-3843.

Chen, Y.-C., Yu, B.-Y., Hsu, C.-C., Chien, I.-L., 2016. Comparison of heteroazeotropic and extractive distillation for the dehydration of propylene glycol methyl ether. Chem. Eng. Res. Des. 111, 184-195.

Chew, J.M., Reddy, C.C.S., Rangaiah, G.P., 2014. Improving energy efficiency of dividing-wall columns using heat pumps, organic rankine cycle and kalina cycle. Chem. Eng. Process.: Process Intensif. 76, 45-59.

Chin, J., Lee, J.W., Choe, J., 2006. Feasible products in complex batch reactive distillation. AIChE J. 52 (5), 1790-1805.

Cordeiro, G.M., De Figueirêdo, M.F., Ramos, W.B., Sales, F.A., Brito, K.D., Brito, R.P., 2017. Systematic strategy for obtaining a dividing-wall column applied to an extractive distillation process. Ind. Eng. Chem. Res. 56 (14), 4083-4094.

Cui, X., Yang, Z., Zhai, Y., Pan, Y., 2002. Batch extractive distillation in a column with a middle vessel. Chin. J. Chem. Eng. 10 (5), 529-534.

Dai, C., Lei, Z., Xi, X., Zhu, J., Chen, B., 2014. Extractive distillation with a mixture of organic solvent and ionic liquid as entrainer. Ind. Eng. Chem. Res. 53 (40), 15786-15791.

De Figueirêdo, M.F., Guedes, B.P., de Araújo, J.M.M., Vasconcelos, L.G.S., Brito, R.P., 2011. Optimal design of extractive distillation columns-a systematic procedure using a process simulator. Chem. Eng. Res. Des. 89, 341-346.

De Figueirêdo, M.F., Brito, K.D., Ramos, W.B., Sales Vasconcelos, L.G., Brito, R.P., 2015a. Effect of solvent content on the separation and the energy consumption of extractive distillation columns. Chem. Eng. Commun. 202 (9), 1191-1199.

De Figueirêdo, M.F., Brito, K.D., Ramos, W.B., Sales Vasconcelos, L.G., Brito, R.P., 2015b. Optimization of the design and operation of extractive distillation processes. Sep. Sci. Technol. 50 (14), 2238-2247.
Dejanović, I., Matijašević, L., Jansen, H., Olujić, Ž, 2011. An effective method for establishing the stage and reflux requirement of three-product dividing wall columns. Chem. Biochem. Eng. Q. 25, 147-157.

Deorukhkar, O.A., Deogharkar, B.S., Mahajan, Y.S., 2016. Purification of tetrahydrofuran from its aqueous azeotrope by extractive distillation: pilot plant studies. Chem. Eng. Process. Process Intensif. 105, 79-91.

Díez, E., Langston, P., Ovejero, G., Romero, M.D., 2009. Economic feasibility of heat pumps in distillation to reduce energy use. Appl. Thermal Eng. 29 (5), 1216-1223.

Diwekar, U., 2012. Batch Distillation: Simulation, Optimal Design, and Control, 2nd edition. CRC Press, $400 \mathrm{p}$.

Doherty, M.F., Caldarola, G.A., 1985. Design and synthesis of homogeneous azeotropic distillations. 3. The sequencing of columns for azeotropic and extractive distillations. Ind. Eng. Chem. Fundam. 24, 474-485.

Doherty, M.F., Malone, M.F., 2001. Conceptual Design of Distillation Systems. McGraw Hill, New York, 568 p.

Dongmin, H., Yanhong, C., 2018. Combining the preconcentration column and recovery column for the extractive distillation of ethanol dehydration with low transition temperature mixtures as entrainers. Chem. Eng. Process.: Process Intensif. 131, 203-214

Duc Long, N., Lee, M., 2013. Optimal retrofit design of extractive distillation to energy efficient thermally coupled distillation scheme. AIChE J. 59 (4), 1175-1182.

Ebrahimzadeh, E., Matagi, J., Fazlollahi, F., Baxter, L.L., 2016. Alternative extractive distillation system for $\mathrm{CO}_{2}$-ethane azeotrope separation in enhanced oil recovery processes. Appl. Therm. Eng. 96, 39-47.

Emhamed, A.M., Czuczai, B., Rev, E., Lelkes, Z., 2008. Analysis of extractive distillation with mathematical programming. Ind. Eng. Chem. Res. 47, 9983-9995.

Errico, M., Rong, B.G., 2012. Synthesis of new separation processes for bioethanol production by extractive distillation. Sep. Purif. Technol. 96, 58-67.

Errico, M., Rong, B.G., Tola, G., Spano, M., 2013. Optimal synthesis of distillation systems for bioethanol separation. Part 1: extractive distillation with simple columns. Ind. Eng. Chem. Res. 52 (4), 1612-1619.

Errico, M., Ramírez-Márquez, C., Torres Ortega, C.E., Rong, B.-G., Segovia-Hernandez, J.G., 2015. Design and control of an alternative distillation sequence for bioethanol purification. J. Chem. Technol. Biotechnol. 90 (12), 2180-2185.

Espinosa, J., 2002. Integration of reaction and separation in a batch extractive distillation column with a middle vessel. Comput. Aided Chem. Eng. 10 (C), 187-192.

Farkas, T., Czuczai, B., Rev, E., Lelkes, Z., 2008. New MINLP Model and Modified Outer Approximation Algorithm for Distillation Column Synthesis. Ind. Eng. Chem. Res. 47, 3088-3103.

Flegiel, F., Sharma, S., Rangaiah, G.P., 2015. Development and multiobjective optimization of improved cumene production processes. Mater. Manuf. Processes 30 (4), 444-457.

Fonyo, Z., Benkö, N., 1998. Comparison of various heat pump assisted distillation configurations. Chem. Eng. Res. Des. 76 (3), 348-360

Frits, E.R., Lelkes, Z., Fonyó, Z., Rév, E., Markót, M.C., Csendes, T., 2006. Finding limiting flows of batch extractive distillation with interval arithmetic. AIChE J. 52, 3100-3108.

Frits, E.R., Markót, M.C., Lelkes, Z., Fonyó, Z., Csendes, T., Rév, E., 2007. Use of an interval global optimization tool for exploring feasibility of batch extractive distillation. J. Global Optim. 38 (2), 297-313.

Gao, X., Zhu, B., Ma, J., Yang, D., 2017. A combination of pressure-swing and extractive distillation for separating complex binary azeotropic system. Chem. Eng. Process.: Process Intensif. 122, 269-276.

García-Herreros, P., Gómez, J.M., Gil, I.D., Rodríguez, G., 2011. Optimization of the design and operation of an extractive distillation system for the production of fuel grade ethanol using glycerol as entrainer. Ind. Eng. Chem. Res. 50 (7), 3977-3985. 
Gerbaud, V., Joulia, X., Rodriguez-Donis, I., Baudouin, O., Rosemain, O., Vacher, A., Castelain, P., 2006. Practical residue curve map analysis applied to solvent recovery in non-ideal binary mixtures by batch distillation processes. Chem. Eng. Process 45 (8), 672-683.

Gerbaud, V., Rodriguez-donis, I., 2014. Extractive distillation. In: Gorak, A., Olujic, Z. (Eds.), Distillation Book, Vol. II Distillation: Equipment and Processes. Elsevier, Amsterdam, pp. 201-246, ISBN 978-0-12-386878-7, Chapter 6

Gil, I.D., Botía, D.C., Ortiz, P., Sánchez, O.F., 2009. Extractive distillation of acetone/methanol mixture using water as entrainer. Ind. Eng. Chem. Res. 48, 4858-4865.

Gil, I.D., Gómez, J.M., Rodríguez, G., 2012. Control of an extractive distillation process to dehydrate ethanol using glycerol as entrainer. Comput. Chem. Eng. 39, 129-142.

Gil, I.D., García, L.C., Rodríguez, G., 2014. Simulation of ethanol extractive distillation with mixed glycols as separating agent. Braz. J. Chem. Eng. 31 (1), 259-270.

Gmehling, J., Möllmann, C., 1998. Synthesis of distillation processes using thermodynamic models and the Dortmund data bank. Ind. Eng. Chem. Res. 37, 3112-3123.

Gmehling, J., Li, J., Schiller, M., 1993. A modified UNIFAC model. 2. Present parameter matrix and results for different thermodynamic properties. Ind. Eng. Chem. Res. 32 (1), 178-193.

Gómez-Castro, F.I., Segovia-Hernández, J.G., Hernández, S., Gutiérrez-Antonio, C., Briones-Ramírez, A., Gamino-Arroyo, Z., 2015. Design of non-equilibrium stage separation systems by a stochastic optimization approach for a class of mixtures. Chem. Eng. Process. 88, 58-69.

Grassi, V.G., 1992. Process design and control of extractive distillation. In: Luyben, W.L. (Ed.), Practical Distillation Control. Van Nostrand Reinhold Press, New York, pp. 370-404, ISBN 978-1-4757-0279-8, Chapter 18.

Gu, J., You, X., Tao, C., Li, J., Shen, W., Li, J., 2018a. Improved design and optimization for separating tetrahydrofuran-water azeotrope through extractive distillation with and without heat integration by varying pressure. Chem. Eng. Res. Des. 133, 303-313

Gu, J., You, X., Tao, C., Li, J., Gerbaud, V., 2018b. Energy-saving reduced pressure extractive distillation with heat integration for separating biazeotropic ternary mixture tetrahydrofuran-methanol-water. Ind. Eng. Chem. Res., http://dx.doi.org/10.1021/acs.iecr.8b03123 (submitted for publication).

Gutiérrez-Guerra, R., Segovia-Hernández, J.G., Hernández, S., 2009. Reducing energy consumption and $\mathrm{CO}_{2}$ emissions in extractive distillation. Chem. Eng. Res. Des. 87, 145-152.

Han, J., Lei, Z., Dong, Y., Dai, C., Chen, B., 2015. Process intensification on the separation of benzene and thiophene by extractive distillation. AIChE J. 61 (12), 4470-4480.

Hegely, L., Available for downloading at http://ethesis.inp-toulouse.fr/archive/00002460/ 2013. Improvement of Batch Distillation Separation of Azeotropic Mixtures. PhD Thesis. Institut National Polytechnique de Toulouse, France.

Hegely, L., Lang, P., 2014. Optimisation of a batch extractive distillation process with off-cut recycle. Chem. Eng. Trans. 45, 1117-1122.

Hegely, L., Lang, P., 2016. Optimization of a batch extractive distillation process with recycling off-cuts. J. Clean. Prod. 136, 99-110.

Hegely, L., Lang, P., Gerbaud, V., 2013. Off-cut recycle for batch and batch extractive distillation separation of a multicomponent azeotropic mixture. Chem. Eng. Trans. 35, 967-972.

Hegely, L., Gerbaud, V., Lang, P., 2014. Generalised model for studying feasibility of heterogeneous extractive batch distillation. Ind. Eng. Chem. Res. 53 (12), 17782-17793.

Hilal, N., Yousef, G., Langston, P., 2001. The reduction of extractive agent in extractive distillation and auto-extractive distillation. Chem. Eng. Process. 41, 673-679.
Hilal, N., Yousef, G., Anabtawi, M.Z., 2002. Operating parameters effect on methanol-acetone separation by extractive distillation. Sep. Sci. Technol. 37 (14), 3291-3303.

Hilmen, E., Skogestad, S., Doherty, M., Malone, M., 1997. Integrated design, operation and control of batch extractive distillation with a middle vessel. In: Proceedings of the AIChE Annual Meeting 1997, Los Angeles, USA, paper No. 201h.

Hilmen, E.K., Kiva, V.N., Skogestad, S., 2002. Topology of ternary VLE diagrams: elementary cells. AIChE J. 48 (4), 752-759.

Hsu, K.Y., Hsiao, Y.C., Chien, I.L., 2010. Design and control of dimethyl carbonate-methanol separation via extractive distillation in the dimethyl carbonate reactive-distillation process. Ind. Eng. Chem. Res. 49 (2), 735-749.

Hua, C., Li, X., Xu, S., Bai, P., 2007. Design and operation of batch extractive distillation with two reboilers. Chin. J. Chem. Eng. $15,286-290$.

Huang, S., Li, W., Li, Y., Ma, J., Shen, C., Xu, C., 2016. Process assessment of distillation using intermediate entrainer: conventional sequences to the corresponding dividing-wall columns. Ind. Eng. Chem. Res. 55 (6), 1655-1666.

Hunek, J., Gal, S., Posel, F., Glavic, P., 1989. Separation of an azeotropic mixture by reverse extractive distillation. AIChE J. 35 (7), 1207-1210.

Jana, A.K., 2010. Heat integrated distillation operation. Appl. Energy 87 (5), 1477-1494.

Jork, C., Kristen, C., Pieraccini, D., Stark, A., Chiappe, C., Beste, Y.A., Arlt, W., 2005. Tailor-made ionic liquids. J. Chem. Thermodyn. 37, 537-558.

Junqueira, T.L., Wolf Maciel, M.R., Maciel Filho, R., 2012. Evaluation of Barros and Wolf efficiency correlations for conventional and extractive distillation columns in bioethanol production process. Sep. Sci. Technol. 47, 1031-1037.

Kim, K.J., Diwekar, U.M., Tomazi, K.G., 2004. Entrainer selection and solvent recycling in complex batch distillation. Chem. Eng. Commun. 191 (12), 1606-1633.

Kiss, A.A., Ignat, R.M., 2012. Innovative single step bioethanol dehydration in an extractive dividing-wall column. Sep. Purif. Technol. 98, 290-297.

Kiss, A.A., Ignat, R.M., 2013. Optimal economic design of an extractive distillation process for bioethanol dehydration. Energy Technol. 1, 166-170.

Kiss, A.A., Olujić, Ž., 2014. A review on process intensification in internally heat-integrated distillation columns. Chem. Eng. Process.: Process Intensif. 86, 125-144.

Kiss, A.A., Suszwalak, D.J.-P.C., 2012. Enhanced bioethanol dehydration by extractive and azeotropic distillation in dividing-wall columns. Sep. Purif. Technol. 86, 70-78.

Kiva, V.N., Hilmen, E.K., Skogestad, S., 2003. Azeotropic phase equilibrium diagrams: a survey. Chem. Eng. Sci. 58, 1903-1953.

Knapp, J.P., Doherty, M.F., 1990. Thermal integration of homogeneous azeotropic distillation sequences. AIChE J. 36 (7), 969-984.

Knapp, J.P., Doherty, M.F., 1994. Minimum entrainer flows for extractive distillation: a bifurcation theoretic approach. AIChE J. 40, 243-268.

Knight, J.R., Doherty, M.F., 1986. Design and synthesis of homogeneous azeotropic distillations 5. columns with non-negligible heat effects. Ind. Eng. Chem. Fundam. 25, 279-289.

Knight, J.R., Doherty, M.F., 1989. Optimal design and synthesis of homogeneous azeotropic distillation sequences. Ind. Eng. Chem. Res. 28, 564-572.

Kossack, S., Kraemer, K., Gani, R., Marquardt, W., 2008. A systematic synthesis framework for extractive distillation processes. Chem. Eng. Res. Des. 86, 781-792.

Kotai, B., Lang, P., 2005. Separation of maximum azeotropes in a middle vessel column. Chem. Eng. Trans. 7, 333-338.

Kotai, B., Lang, P., Modla, G., 2007. Batch extractive distillation as a hybrid process: separation of minimum boiling azeotropes. Chem. Eng. Sci. 62, 6816-6826.

Kraemer, K., Kossack, S., Marquardt, W., 2007. An efficient solution method for the MINLP optimization of chemical 
processes, in: Pleşu, V., Agachi, P.S. (Eds.). Comput. Aided Chem. Eng. 24, Elsevier, Amsterdam, 105-110.

Kumar, S., Wright, J.D., Taylor, P.A., 1984. Modelling and dynamics of an extractive distillation column. Can. J. Chem. Eng. 62 (6), 780-789.

Lang, P., 1992. Computation of multistage multicomponent separation processes. In: Pallai, I., Fonyo, Z.s. (Eds.), Studies in Computer-aided Modelling, Design and Operation: Unit Operations Pt. A Operations. Elsevier, London, pp. 256-399, ISBN: 978-0444986733. Chapter 7.

Lang, P., Yatim, H., Moszkowicz, P., Otterbein, M., 1994. Batch extractive distillation under constant reflux ratio. Comput. Chem. Eng. 18, 1057-1062

Lang, P., Lelkes, Z., Moszkowicz, P., Otterbein, M., Yatim, H., 1995. Different operational policies for the batch extractive distillation. Comput. Chem. Eng. 19, S645-S650.

Lang, P., Lelkes, Z., Otterbein, M., Benadda, B., Modla, G., 1999. Feasibility studies for batch extractive distillation with a light entrainer. Comput. Chem. Eng. 23, S93-98.

Lang, P., Modla, G., Benadda, B., Lelkes, Z., 2000a. Homoazeotropic distillation of maximum azeotropes in a batch rectifying column with continuous entrainer feeding. I. Feasibility studies. Comput. Chem. Eng. 24, 1665-1671.

Lang, P., Modla, G., Kotai, B., Lelkes, Z., Moszkowicz, P., 2000 b. Homoazeotropic distillation of maximum azeotropes in a batch rectifying column with continuous entrainer feeding. II. Rigorous simulation results. Comput. Chem. Eng. 24, 1429-1435.

Lang, P., Kovacs, G.y., Kotai, B., Gaal-Szilagyi, J., Modla, G., 2006. Industrial application of a new batch extractive distillation operational policy. Inst. Chem. Eng. Symp. Ser. 152, 830-839.

Lang, P., Hegely, L., Kovacs, G.y., Gaal-Szilagyi, J., Kotai, B., 2010. Solvent recovery from a multicomponent mixture by batch extractive distillation and hybrid process. In: de Haan, A., Kooijman, H. (Eds.), Distillation, Absorption 2010: Conference Proceedings. Eindhoven University of Technology, Eindhoven, Netherlands, pp. 295-300.

Lang, P., Hegely, L., Kovacs, G., 2012. Method for the recovery of methanol from multicomponent solvent mixtures, P 1200245 , Hungarian Patent.

Langston, P., Hilal, N., Shingfield, S., Webb, S., 2005. Simulation and optimisation of extractive distillation with water as solvent. Chem. Eng. Process. 44, 345-351.

Laroche, L., Bekiaris, N., Andersen, H.W., Morari, M., 1991. Homogeneous azeotropic distillation: comparing entrainers. Can. J. Chem. Eng. 69, 1302-1319.

Laroche, L., Bekiaris, N., Andersen, H.W., Morari, M., $1992 a$. Homogeneous azeotropic distillation: separation and flowsheet synthesis. Ind. Eng. Chem. Eng. 31, 2190-2209.

Laroche, L., Bekiaris, N., Andersen, H.W., Morari, M., 1992b. The curious behavior of homogeneous azeotropic distillation-implications for entrainer selection. AIChE J. 38, 1309-1328.

Leboreiro, J., Acevedo, J., 2004. Processes synthesis and design of distillation sequences using modular simulators: a genetic algorithm framework. Comput. Chem. Eng. 28 (8), 1223-1236.

Lee, F.M., Pahl, R.H., 1985. Solvent screening study and conceptual extractive distillation process to produce anhydrous ethanol from fermentation broth. Ind. Eng. Chem. Proc. Dev. 24, 168-172.

Lei, Z., Wang, H., Zhou, R., Duan, Z., 2002. Process improvement on separating C4 by extractive distillation. Chem. Eng. J. 87, 379-386.

Lei, Z., Li, C., Chen, B., 2003. Extractive distillation: a review. Sep. Purif. Rev. 32 (2), 121-213.

Lei, Z., Dai, C., Zhu, J., Chen, B., 2014. Extractive distillation with ionic liquids: a review. AIChE J. 60 (9), 3312-3329.

Lek-Utaiwan, P., Suphanit, B., Douglas, P.L., Mongkolsiri, N., 2011. Design of extractive distillation for the separation of close-boiling mixtures: solvent selection and column optimization. Comput. Chem. Eng. 35 (6), 1088-1100.
Lelkes, Z., Lang, P., Benadda, B., Moszkowicz, P., 1998a. Feasibility of extractive distillation in a batch rectifier. AIChE J. 44 (4), 810-822.

Lelkes, Z., Lang, P., Moszkowicz, P., Benadda, B., Otterbein, M., 1998b. Batch extractive distillation: the process and the operational policies. Chem. Eng. Sci. 53 (7), 1331-1348.

Lelkes, Z., Lang, P., Otterbein, M., 1998c. Feasibility and sequencing studies for homoazeotropic distillation in a rectifier with continuous entrainer feeding. Comput. Chem. Eng. 22, S653-S656.

Lelkes, Z., Rev, E., Steger, C., Fonyo, Z., 2002. Batch extractive distillation of maximal azeotrope with middle boiling entrainer. AIChE J. 48 (11), 2524-2536.

Lelkes, Z., Rev, E., Steger, C., Varga, V., Fonyo, Z., Horvath, L., 2003a. Batch extractive distillation with intermediate boiling entrainer. Comput. Aided Chem. Eng. 14, 197-202.

Lelkes, Z., Rodriguez-Donis, I., Lovász, A., Papp, K., Gerbaud, V., Rév, E., 2003b. Feasibility studies of heterogeneous extractive distillation process. In: Proc. of Days of Chem. Eng. '03, Veszprém, Hungary, April 8th-10th, 2003, pp. 208-213, ISBN 9637172998.

Lelkes, Z., Szitkai, Z., Farkas, T., Rev, E., Fonyo, Z., 2003c. Short-cut design of batch extractive distillation using MINLP. Comput. Aided Chem. Eng. 14, 203-208.

Levy, S.G., Doherty, M.F., 1986. Design and synthesis of homogeneous azeotropic distillations. 4. Minimum reflux calculations for multiple-feed columns. Ind. Eng. Chem. Fundam. 25 (2), 269-279.

Levy, S.G., Van Dongen, D.B., Doherty, M.F., 1985. Design and synthesis of homogeneous azeotropic distillations. 2 Minimum reflux calculations for nonideal and azeotropic columns. Ind. Eng. Chem. Fundam. 24 (4), 463-474.

Li, G., Bai, P., 2012. New operation strategy for separation of ethanol-water by extractive distillation. Ind. Eng. Chem. Res. 51, 2723-2729.

Li, J.-H., Bai, P., Hua, C., Zhang, Z.-G., 2006. Middle-vessel batch extractive distillation with mixed solvents. J. Tianjin Univ. Sci. Technol. 39 (7), 881-884.

Li, H., Wu, Y., Li, X., Gao, X., 2016. State-of-the-art of advanced distillation technologies in China. Chem. Eng. Technol. 39 (5), 815-833.

Li, L., Guo, L., Tu, Y., Yu, N., Sun, L., Tian, Y., Li, Q., 2017. Comparison of different extractive distillation processes for 2-methoxyethanol/toluene separation: design and control. Comput. Chem. Eng. 99, 117-134.

Liang, K., Li, W., Luo, H., Xia, M., Xu, C., 2014. Energy-efficient extractive distillation process by combining preconcentration column and entrainer recovery column. Ind. Eng. Chem. Res. 53 (17), 7121-7131.

Liang, S., Cao, Y., Liu, X., Li, X., Zhao, Y., Wang, Y., Wang, Y., 2017. Insight into pressure-swing distillation from azeotropic phenomenon to dynamic control. Chem. Eng. Res. Des. 117, 318-335.

Liu, H.X., Wang, N., Zhao, C., Ji, S., Li, J.R., 2017. Membrane materials in the pervaporation separation of aromatic/aliphatic hydrocarbon mixtures-a review. Chin. J. Chem. Eng. 26 (1), 1-16.

Lo, K.M., Chien, I.L., 2017. Efficient separation method for tert-butanol dehydration via extractive distillation. J. Taiwan Inst. Chem. Eng. 73, 27-36.

Low, K.H., Sorensen, E., 2002. Optimal operation of extractive distillation in different batch configurations. AIChE J. 48 (5), 1034-1050.

Lucia, A., Amale, A., Taylor, R., 2008. Distillation pinch points and more. Comput. Chem. Eng. 32, 1350-1372.

Luo, H., Liang, K., Li, W., Li, Y., Xia, M., Xu, C., 2014. Comparison of pressure-swing distillation and extractive distillation methods for isopropyl alcohol/diisopropyl ether separation. Ind. Eng. Chem. Res. 53 (39), 15167-15182.

Luo, H., Bildea, C.S., Kiss, A.A., 2015. Novel heat-pump-assisted extractive distillation for bioethanol purification. Ind. Eng. Chem. Res. 54, 2208-2213. 
Luyben, W.L., 1988. Multicomponent batch distillation. 1: Ternary systems with slop recycle. Ind. Eng. Chem. Res. 27, 642-647.

Luyben, W.L., 2006. Plantwide control of an isopropyl alcohol dehydration process. AIChE J. 52, 2290-2296.

Luyben, W.L., 2008a. Effect of solvent on controllability in extractive distillation. Ind. Eng. Chem. Res. 47, 4425-4439.

Luyben, W.L., 2008b. Comparison of extractive distillation and pressure-swing distillation for acetone-methanol separation. Ind. Eng. Chem. Res. 47, 2696-2707.

Luyben, W.L., 2013. Comparison of extractive distillation and pressure-swing distillation for acetone/chloroform separation. Comput. Chem. Eng. 50, 1-7.

Luyben, W.L., 2015. Improved design of an extractive distillation system with an intermediate-boiling solvent. Sep. Purif. Technol. 156, 336-347.

Luyben, W.L., 2016a. Control comparison of conventional extractive distillation with a new split-feed configuration. Chem. Eng. Process.: Process Intensif. 107, 29-41.

Luyben, W.L., 2016b. Distillation column pressure selection. Sep. Purif. Technol. 168, 62-67.

Luyben, W.L., 2016c. Control comparison of conventional and thermally coupled ternary extractive distillation processes. Chem. Eng. Res. Des. 107, 29-41.

Luyben, W.L., 2017. Improved plantwide control structure for extractive divided-wall columns with vapor recompression. Chem. Eng. Res. Des. 123, 152-164.

Luyben, W.L., 2018a. Control of heat-integrated extractive distillation processes. Comput. Chem. Eng. 111, 267-277.

Luyben, W.L., 2018b. Vapor split manipulation in extractive divided-wall distillation columns. Chem. Eng. Process.: Process Intensif. 126, 132-140.

Luyben, W.L., Chien, I.L., 2010. Design and Control of Distillation Systems for Separating Azeotropes. Wiley-VCH, New York, 453 p.

Lynn, S., Hanson, D.N., 1986. Multieffect extractive distillation for separating aqueous azeotropes. Ind. Eng. Chem. Process Des. Dev. 25, 936.

Matsuyama, H., Nishimura, H., 1977. Topological and thermodynamic classification of ternary vapor-liquid equilibria. J. Chem. Eng. Jpn. 10 (3), 181-187.

Medina-Herrera, N., Grossmann, I.E., Mannan, M.S., Jiménez-Gutiérrez, A., 2014. An approach for solvent selection in extractive distillation systems including safety considerations. Ind. Eng. Chem. Res. 53, 12023-12031.

Midori, S., Zheng, S.N., Yamada, I., 2000. Analysis of divided-wall column for extractive distillation. Kagaku Kogaku Ronbunshu 26 (5), 627-632 (in Japanese).

Milani, S.M., 1999. Optimisation of solvent feed rate for maximum recovery of high purity top product in batch extractive distillation. Chem. Eng. Res. Des. 77, 469-470.

Modla, G., 2013. Energy saving methods for separation of a minimum boiling point azeotrope using intermediate entrainer. Energy 50 (1), 103-109.

Modla, G., Lang, P., 2012. Removal and recovery of organic solvents from aqueous waste mixtures by extractive and pressure swing distillation. Ind. Eng. Chem. Res. 51 (35), 11473-11481.

Modla, G., Lang, P., 2013. Heat pump systems with mechanical compression for batch distillation. Energy 62, 403-417.

Modla, G., Lang, P., Molnar, K., 2001. Batch heteroazeotropic rectification of a low relative volatility mixture under continuous entrainer feeding: feasibility studies, CD-rom. In: Proceedings of 6th World Congress of Chemical Engineering, Melbourne, Australia.

Modla, G., Lang, P., Kótai, B., Molnár, K., 2003. Batch heteroazeotropic rectification of a low relative volatility mixture under continuous entrainer feeding. AIChE J. 49 (10), 2533-2552.

Momoh, S.O., 1991. Assessing the accuracy of selectivity as a basis for solvent screening in extractive distillation processes. Sep. Purif. Technol. 26, 729-742.
Moraru, M.D., Bildea, C.S., 2017. Design and plantwide control of n-butyl acrylate production process. J. Process Control 58, 46-62.

Mujtaba, I.M., 1999. Optimization of batch extractive distillation processes for separating close boiling and azeotropic mixtures. Chem. Eng. Res. Des. 77, 588-596.

Mujtaba, I.M., 2004. Batch Distillation. Design and Operation, Series on Chemical Engineering, Vol. 3. Imperial College Press, London, $450 \mathrm{p}$.

Munoz, R., Monton, J.B., Burguet, M.C., de la Torre, J., 2006. Separation of isobutyl alcohol and isobutyl acetate by extractive distillation and pressure-swing distillation: simulation and optimization. Sep. Purif. Technol. 50 (2), 175-183.

Pacheco-Basulto, J.A., Hernández-McConville, D., Barroso-Muñoz, F.O., Hernández, S., Segovia-Hernández, J.G., Castro-Montoya, A.J., Bonilla-Petriciolet, A., 2012. Purification of bioethanol using extractive batch distillation: simulation and experimental studies. Chem. Eng. Process. 61, 30-35.

Palacios-Bereche, R., Ensinas, A.V., Modesto, M., Nebra, S.A., 2015. Double-effect distillation and thermal integration applied to the ethanol production process. Energy 82, 512-523.

Patrascu, I., Bildea, 2017. Process control of a heat pump assisted extractive DWC for bioethanol dehydration. Comput. Aided Chem. Eng. 40, 1549-1554.

Patrascu, I., Bildea, C.S., Kiss, A.A., 2017. Dynamics and control of a heat-pump assisted extractive dividing-wall column for bioethanol dehydration. Chem. Eng. Res. Des. 119, 66-74.

Pereiro, A.B., Araújo, J.M.M., Esperança, J.M.S.S., Marrucho, I.M., Rebelo, L.P.N., 2012. Ionic liquids in separations of azeotropic systems-a review. J. Chem. Thermodyn. 46, 2-28.

Peng, Y., Lu, X., Liu, B., Zhu, J., 2017. Separation of azeotropic mixtures (ethanol and water) enhanced by deep eutectic solvents. Fluid Phase Equilib. 448, 128-134.

Peterson, E.J., Partin, L.R., 1997. Temperature sequences for categorizing all ternary distillation boundary maps. Ind. Eng. Chem. Res. 36 (5), 1799-1811.

Petlyuk, F.B., 2004. Distillation Theory and Its Application to Optimal Design of Separation Units. Cambridge Series in Chemical Engineering. Cambridge University Press, Cambridge, UK, 362 p.

Petlyuk, F.B., Danilov, R.Yu., 1999. Sharp distillation of azeotropic mixtures in a two-feed column. Theor. Found. Chem. Eng. 33, 233-242.

Petlyuk, F., Danilov, R., Burger, J., 2015. A novel method for the search and identification of feasible splits pf extractive distillations in ternary mixtures. Chem. Eng. Res. Des. 99, 132-148.

Pradhan, S., Kannan, A., 2005. Simulation and analysis of extractive distillation process in a valve tray column using the rate based model. Korean J. Chem. Eng. 22 (3), 441-451.

Pretel, E.J., López, P.A., Bottini, S.B., Brignole, E.A., 1994. Computeróaided molecular design of solvents for separation processes. AIChE J. 40 (8), 1349-1360.

Quijada-Maldonado, E., Aelmans, T.A.M., Meindersma, G.W., de Haan, A.B., 2013. Pilot plant validation of a rate-based extractive distillation model for water-ethanol separation with the ionic liquid [emim][DCA] as solvent. Chem. Eng. J. 223, 287-297.

Quijada-Maldonado, E., Meindersma, G.W., de Haan, A.B., 2016. Pilot plant study on the extractive distillation of toluene-methylcyclohexane mixtures using NMP and the ionic liquid [hmim][TCB] as solvents. Sep. Purif. Technol. 166, 196-204.

Raeva, V.M., Sazonova, A.Y., 2015. Separation of ternary mixtures by extractive distillation with 1,2-ethanediol and glycerol. Chem. Eng. Res. Des. 99, 125-131.

Raeva, V.M., Sazonova, A.Y., Frolkova, A.K., 2013. Synergetic effect of binary separating agents in extractive rectification of homogeneous mixtures. Theor. Found. Chem. Eng. 47 (5), 649-659.

Ramos, M.A., García-Herreros, P., Gomez, J.M., Reneaume, J.M., 2013. Optimal control of the extractive distillation for the 
production of fuel-grade ethanol. Ind. Eng. Chem. Res. 52, 8471-8487.

Ramos, M.A., Gómez, J.M., Reneaume, J.M., 2014. Simultaneous optimal design and control of an extractive distillation system for the production of fuel grade ethanol using a mathematical program with complementarity constraints. Ind. Eng. Chem. Res. 53 (2), 752-764.

Ravagnani, M.A.S.S., Reis, M.H.M., Maciel Filho, R., Wolf-Maciel, M.R., 2010. Anhydrous ethanol production by extractive distillation: a solvent case study. Process Saf. Environ. Prot. 88, 67-73.

Reddy, C.C.S., Fang, Y., Rangaiah, G.P., 2014. Improving energy efficiency of distillation using heat pump assisted columns. Asia Pacific J. Chem. Eng. 9 (6), 905-928.

Repke, J.U., Mertens, P., Arellano-Garcia, H., Lelkes, Z., Rev, E., Wozny, G., 2007. Modelling and simulation of start-up strategies for batch extractive distillation. Inz. Chem. Procesowa 28 (1), 149-160.

Resa, J.M., González, C., Ruiz, A., 2000. Experiments of extractive distillation at laboratory scale for the rupture of the azeotropic mixture acetone + isopropyl ether. Sep. Purif. Technol. 18, 103-110.

Reshetov, S.A., Kravchenko, S.V., 2007. Statistics of liquid-vapor phase equilibrium diagrams for various ternary zeotropic mixtures. Theor. Found. Chem. Eng. 41 (4), 451-453.

Rev, E., Lelkes, Z., Varga, V., Steger, C., Fonyo, Z., 2003. Separation of a minimum-boiling azeotrope in a batch extractive rectifier with an intermediate-boiling entrainer. Ind. Eng. Chem. Res. 42, 162-174.

Rodriguez, N.R., Kroon, M.C., 2015. Isopropanol dehydration via extractive distillation using low transition temperature mixtures as entrainers. J. Chem. Thermodyn. 85, 216-221.

Rodríguez-Donis, I., Gerbaud, V., Joulia, X., 2002. Feasibility of heterogeneous batch distillation processes. AIChE J. 48 (6), 1168-1178.

Rodríguez-Donis, I., Acosta-Esquijarosa, J., Gerbaud, V., Joulia, X., 2003. Heterogeneous batch extractive distillation of minimum boiling azeotropic mixtures. AIChE J. 49 (12), 3074-3083.

Rodríguez-Donis, I., Papp, K., Gerbaud, V., Joulia, X., Rev, E., Lelkes, Z., 2007. Column configurations of continuous heterogeneous extractive distillation. AIChE J. 53 (8), 1982-1993.

Rodríguez-Donis, I., Gerbaud, V., Joulia, X., 2009a. Thermodynamic insights on the feasibility of homogeneous batch extractive distillation. 1. Azeotropic mixtures with heavy entrainer. Ind. Chem. Eng. Res. 48 (7), 3544-3559.

Rodríguez-Donis, I., Gerbaud, V., Joulia, X., 2009b. Thermodynamic insights on the feasibility of homogeneous batch extractive distillation, 2 . low-relative-volatility binary mixtures with a heavy entrainer. Ind. Chem. Eng. Res. 48 (7), 3560-3572.

Rodríguez-Donis, I., Gerbaud, V., Joulia, X., 2010. Thermodynamic insight on extractive distillation with entrainer forming new azeotropes. In: de Haan, A., Kooijman, H. (Eds.), Distillation, Absorption 2010: Conference Proceedings. Eindhoven University of Technology, Eindhoven, Netherlands, pp. 431-436.

Rodríguez-Donis, I., Gerbaud, V., Joulia, X., 2012a.

Thermodynamic insights on the feasibility of homogeneous batch extractive distillation. 3. Azeotropic mixtures with light boiling entrainer. Ind. Chem. Eng. Res. 51, 4643-4660.

Rodríguez-Donis, I., Gerbaud, V., Joulia, X., 2012b. Thermodynamic insights on the feasibility of homogeneous batch extractive distillation, 4. azeotropic mixtures with intermediate boiling entrainer. Ind. Chem. Eng. Res. 51, 6489-6501.

Rodríguez, N.R., González, A.S.B., Tijssen, P.M.A., Kroon, M.C., 2015. Low transition temperature mixtures (LTTMs) as novel entrainers in extractive distillation. Fluid Phase Equilib. 385, 72-78.

Safrit, B.T., Westerberg, A.W., 1997a. Improved operational policies for batch extractive distillation columns. Ind. Eng. Chem. Res. 36 (2), 436-443.
Safrit, B.T., Westerberg, A.W., 1997b. Synthesis of azeotropic batch distillation separation systems. Ind. Eng. Chem. Res. 36 (5), 1841-1854.

Safrit, B.T., Westerberg, A.W., Diwekar, U.M., Wahnschafft, O.M., 1995. Extending continuous conventional and extractive distillation feasibility insights to batch distillation. Ind. Eng. Chem. Res. 34, 3257-3264.

Sauar, E., Siragusa, G., Andresen, B., 2001. Equal thermodynamic distance and equipartition of forces principles applied to binary distillation. J. Phys. Chem. A 105, 2312-2320.

Sazonova, A.Y., Raeva, V.M., 2015. Recovery of acetonitrile from aqueous solutions by extractive distillation-effect of entrainer. Int. J. Chem. Mol. Nucl. Mater. Metall. Eng. 9 (2), 288-291.

Sazonova, A.Y., Raeva, V.M., Chelyuskina, T.V., Frolkova, A.K., 2014. Criterion selecting potential separating agents for extractive distillation. Theor. Found. Chem. Eng. 48 (2), 148-157

Sazonova, A.Y., Raeva, V.M., Frolkova, A.K., 2016. Design of extractive distillation process with mixed entrainer. Chem. Papers 70 (5), 594-601.

Schneibel, J. Absolute Alcohol. U.S. Patent 1,469,447, Oct 2, 1923.

Segovia-Hernández, J.G., Vázquez-Ojeda, M., Gómez-Castro, F.I., Ramírez-Márquez, C., Errico, M., Tronci, S., Rong, B.-G., 2014. Process control analysis for intensified bioethanol separation systems. Chem. Eng. Process.: Process Intensif. 75, 119-125.

Segovia-Hernández, J.G., Hernández, S., Bonilla Petriciolet, A., 2015. Reactive distillation: a review of optimal design using deterministic and stochastic techniques. Chem. Eng. Res. Des. 97, 134-143.

Seider, J.D., Sirola, J.J., Barnicki, S.D., 1997. Distillation. Chapter 13. In: Perry, R.H., Green, D.W. (Eds.), Perry's Chemical Engineers' Handbook. , 7th edition. McGraw-Hill, New York, pp. 13-79, ISBN 978-0070498419.

Serafimov, L.A., 1970. The azeotropic rule and the classification of multicomponent mixtures VII. diagrams for ternary mixtures. Russ. J. Phys. Chem. 44, 567-571.

Serafimov, L.A., 1996. Thermodynamic and topological analysis of liquid-vapor phase equilibrium diagrams and problems of rectification of multicomponent mixtures. In: Kuchanov, S.I. (Ed.), Mathematical Methods in Contemporary Chemistry. Gordon and Breach Publishers, Amsterdam, pp. 557-605.

Serafimov, L.A., Frolkova, A.K., Bushina, D.I., 2008. Extractive distillation of binary azeotropic mixtures. Theor. Found. Chem. Eng. 42, 507-516.

Shcherbakova, N.N., Rodriguez-Donis, I., Gerbaud, V., 2015. On the Riemanian structure of residue curve maps. Chem. Eng. Res. Des. 99, 87-96.

Shcherbakova, N.N., Rodriguez-Donis, I., Abildskov, J., Gerbaud, V., 2017. A novel method for detecting and computing univolatility curves in ternary mixtures. Chem. Eng. Sci. 173, 21-36.

Shen, W., Available for downloading at http://ethesis.inp-toulouse.fr/archive/00002137/ 2012. Extension of Thermodynamic Insights on Batch Extractive Distillation to Continuous Operation. PhD Thesis. Institut National Polytechnique de Toulouse, Toulouse.

Shen, W., Gerbaud, V., 2013. Extension of thermodynamic insights on batch extractive distillation to continuous operation. 2. Azeotropic mixtures with a light entrainer. Ind. Eng. Chem. Res. 52 (12), 4623-4637.

Shen, W., Benyounes, H., Gerbaud, V., 2013. Extension of thermodynamic insights on batch extractive distillation to continuous operation. 1. Azeotropic mixtures with a heavy entrainer. Ind. Eng. Chem. Res. 52 (12), 4606-4622.

Shen, W., Dong, L., Wei, S., Li, J., Benyounes, H., You, X., Gerbaud, V., 2015a. Systematic design of an extractive distillation for maximum-boiling azeotropes with heavy entrainers. AIChE J. 61 (11), 3898-3910.

Shen, W.F., Benyounes, H., Song, J., 2015b. Thermodynamic topological analysis of extractive distillation of maximum boiling azeotropes. Braz. J. Chem. Eng. 32 (4), 957-966. 
Shen, W., Benyounes, H., Gerbaud, V., 2015c. Extractive distillation: recent advances in operation strategies. Rev. Chem. Eng. 31 (1), 13-26.

Shen, W.F., Benyounes, H., Dong, L.C., Wei, S.A., Li, J., Gerbaud, V., 2016. Conceptual design of non-ideal mixture separation with light entrainers. Braz. J. Chem. Eng. 33 (4), 1041-1053.

Skiborowski, M., Rautenberg, M., Marquardt, W., 2015. A hybrid evolutionary-deterministic optimization approach for conceptual design. Ind. Eng. Chem. Res. 51 (41), 10054-10072.

Souto, R.M.L.O., Wanderley Farias Neto, G., de Araújo, F.S., De Figueirêdo, M.F., Ramos, W.B., Brito, K.D., Brito, R.P., 2018. Rigorous thermodynamic evaluation of the extractive distillation process. Chem. Eng. Res. Des. 133, 195-203.

Staak, D., Grützner, T., 2017. Process integration by application of an extractive dividing-wall column: an industrial case study. Chem. Eng. Res. Des. 123, 120-129.

Stéger, C., Available for downloading at http://ethesis.inp-toulouse.fr/archive/00000358/2006. Batch Extractive and Reactive Distillation in Middle Vessel Column. Ph.D. Thesis. Institut National Polytechnique de Toulouse, Toulouse.

Stéger, C., Varga, V., Horvath, L., Rev, E., Fonyo, Z., Meyer, M., Lelkes, Z., 2005. Feasibility of extractive distillation process variants in batch rectifier column. Chem. Eng. Process. 44, 1237-1256

Stéger, C., Rév, E., Horváth, L., Fonyó, Z., Meyer, M., Lelkes, Z., 2006. New extractive configuration separating azeotropic mixture in semi-batch way. Sep. Purif. Technol. 52, 343-356.

Stichlmair, J.G., Fair, J.R., 1998. Distillation: Principles and Practices. Wiley-VCH, New York, 544 p ISBN: 978-0-471-25241-2

Sun, L., Wang, Q., Li, L., Zhai, J., Liu, Y., 2014. Design and control of extractive dividing wall column for separating benzene/cyclohexane mixtures. Ind. Eng. Chem. Res. 53 (19), 8120-8131.

Tavan, Y., Shahhosseini, S., Hosseini, S.H., 2014. Design and simulation of ethane recovery process in an extractive dividing wall column. J. Clean. Prod. 72, 222-229.

Taylor, M., Wankat, P.C., 2004. Increasing the energy efficiency of extractive distillation. Sep. Sci. Technol. 39 (1), 1-17.

Timoshenko, A.V., 2004. Synthesis of distillation flowsheets with fully coupled heat and material flows using graph theory. Theor. Found. Chem. Eng. 38 (3), 251-254.

Timoshenko, A.V., Serafimov, L.A., 2001. Flowsheet synthesis strategy for irreversible zeotropic distillation. Theor. Found. Chem. Eng. 35 (6), 567-572.

Timoshenko, A.V., Patkina, O.D., Serafimov, L.A., 2001. Synthesis of optimal distillation flowsheets consisting of columns with various numbers of sections. Theor. Found. Chem. Eng. 35 (5), 458-464.

Timoshenko, A., Anokhina, E., Ivanova, L., 2005. Extractive distillation systems involving complex columns with partially coupled heat and material flows. Theor. Found. Chem. Eng. 39, 463-470.

Timoshenko, A.V., Anokhina, E.A., Morgunov, A.V., Rudakov, D.G., 2015. Application of the partially thermally coupled distillation flowsheets for the extractive distillation of ternary azeotropic mixtures. Chem. Eng. Res. Des. 104, 139-155.

Tiverios, P.G., van Brunt, V., 2000. Extractive distillation solvent characterization and shortcut design procedure for methylcyclohexane-toluene mixtures. Ind. Eng. Chem. Res. 39, 1614-1623.

Tondeur, D., Kvaalen, E., 1987. Equipartition of entropy production. An optimality criterion for transfer and separation processes. Ind. Eng. Chem. Res. 26, 50-56.

Torres-Ortega, C.E., Segovia-Hernández, J.G., Gómez-Castro, F.I., Hernández, S., Bonilla-Petriciolet, A., Rong, B.-G., Errico, M., 2014. Design, optimization and controllability of an alternative process based on extractive distillation for an ethane-carbon dioxide mixture. Chem. Eng. Process.: Process Intensif. 74, 55-68.
Tututi-Avila, S., Jimenez-Gutierrez, A., Hahn, J., 2014. Control analysis of an extractive dividing-wall column used for ethanol dehydration. Chem. Eng. Process. 82, 88-100.

Tututi-Avila, S., Medina-Herrera, N., Hahn, J., Jiménez-Gutiérrez, A., 2017. Design of an energy-efficient side-stream extractive distillation system. Comput. Chem. Eng. 102, 17-25.

Van de Bor, D.M., Ferreira, C.A.I., 2013. Quick selection of industrial heat pump types including the impact of thermodynamic losses. Energy 53, 312-322.

Van Dongen, D.B., Doherty, M.F., 1985a. Design and synthesis of homogeneous azeotropic distillations. 1 Problem formulation for a single column. Ind. Eng. Chem. Fundam. 24 (4), 454-463.

Van Dongen, D.B., Doherty, M.F., 1985b. On the dynamics of distillation processs. VI. Batch distillation. Chem. Eng. Sci. 40, 2087-2093.

Van Kaam, R., Rodriguez-Donis, I., Gerbaud, V., 2008. Heterogeneous extractive batch distillation of chloroform-methanol-water: feasibility and experiments. Chem. Eng. Sci. 63, 78-94.

Varga, V., Available for downloading at http://ethesis.inp-toulouse.fr/archive/00000406/ 2006. Distillation Extractive Discontinue dans une Colonne de Rectification et dans une Colonne Inverse./Batch extractive distillation in a rectifier and in a stripper. Ph.D. Thesis. Institut National Polytechnique de Toulouse, Toulouse.

Varga, V., Frits, E.R., Gerbaud, V., Fonyo, Z., Joulia, X., Lelkes, Z., Rev, E., 2006b. Separation of azeotropes in batch extractive stripper with intermediate entrainer. Comput. Aided Chem. Eng. 21 (1), 793-797.

Varga, V., Rev, E., Gerbaud, V., Lelkes, Z., Fonyo, Z., Joulia, X., 2006a. Batch extractive distillation with light entrainer. Chem. Biochem. Eng. Q. 20 (1), 1-24.

Wahnschafft, O.M., Westerberg, A.W., 1993. The product composition regions of azeotropic distillation columns: 2. separability in two-feed columns and entrainer selection. Ind. Eng. Chem. Res. 32, 1108-1120.

Wang, Q., Yu, B., Xu, C., 2012. Design and control of distillation system for methylal/methanol separation. Part 1: extractive distillation using DMF as an entrainer. Ind. Eng. Chem. Res. 51 (3), 1281-1292.

Wang, Y., Cui, P., Ma, Y., Zhang, Z., 2015a. Extractive distillation and pressure-swing distillation for THF/ethanol separation. J. Chem. Technol. Biotechnol. 90 (8), 1463-1472.

Wang, Y., Liang, S., Bu, G., Liu, W., Zhang, Z., Zhu, Z., 2015b. Effect of solvent flow rates on controllability of extractive distillation for separating binary azeotropic mixture. Ind. Eng. Chem. Res. 54, 12908-12919.

Wang, Y., Zhang, Z., Zhao, Y., Liang, S., Bu, G., 2015c. Control of extractive distillation and partially heat-integrated pressure-swing distillation for separating azeotropic mixture of ethanol and tetrahydrofuran. Ind. Eng. Chem. Res. 54 (34), 8533-8545.

Wang, H., Li, Y., Su, W., Zhang, Y., Guo, J., Li, C., 2016. Design and control of extractive distillation based on an effective relative gain array. Chem. Eng. Technol. 39 (12), 2339-2347.

Wang, Q., Chen, J.Y., Pan, M., He, C., He, C.H., Zhang, B.J., Chen, Q.L., 2018a. A new sulfolane aromatic extractive distillation process and optimization for better energy utilization. Chem. Eng. Process.: Process Intensif. 128, 80-95.

Wang, Y., Zhang, X., Liu, X., Bai, W., Zhu, Z., Wang, Y., Gao, J., 2018b. Control of extractive distillation process for separating heterogenerous ternary azeotropic mixture via adjusting the solvent content. Sep. Purif. Technol. 191, 8-26.

Warter, M., Stichlmair, J., 1999. Batchwise extractive distillation in a column with a middle vessel. Comput. Chem. Eng. 23, S915-S918.

Warter, M., Demicoli, D., Stichlmair, J., 2004. Operation of a batch distillation column with a middle vessel: experimental results for the separation of zeotropic and azeotropic mixtures. Chem. Eng. Process.: Process Intensif. 43 (3), 263-272.

Weiss, S., Arlt, R., 1987. On the modelling of mass transfer in extractive distillation. Chem. Eng. Process.: Process Intensif. 21 (2), 107-113. 
Widagdo, S., Seider, W.D., 1996. Azeotropic distillation. AIChE J. 42, 96-130.

Wu, L.L., Chang, W.X., Guan, G.F., 2007. Extractants design based on an improved genetic algorithm. Ind. Eng. Chem. Res. 46, 1254-1258.

Wu, Y.C., Hsu, P.H.-C., Chien, I.-L., 2013. Critical assessment of the energy-saving potential of an extractive dividing-wall column. Ind. Eng. Chem. Res. 52 (15), 5384-5399.

Xia, M., Yu, B., Wang, Q., Jiao, H., Xu, C., 2012. Design and control of extractive dividing-wall column for separating methylal-methanol mixture. Ind. Eng. Chem. Res. 51, 16016-16033.

Xu, S., Wang, H., 2006. Separation of tetrahydrofuran-water azeotropic mixture by batch extractive distillation process Chem. Eng. Res. Des. 84, 478-482.

Yang, A., Wei, R., Sun, S., Wei, S., Shen, W., Chien, I.L., 2018. Energy-saving optimal design and effective control of heat integration-extractive dividing wall column for separating heterogeneous mixture methanol/toluene/water with multiazeotropes. Ind. Eng. Chem. Res. 57, 8036-8056.

Yao, J.Y., Lin, S.Y., Chien, I.L., 2007. Operation and control of batch extractive distillation for the separation of mixtures with minimum-boiling azeotrope. J. Chin. Inst. Chem. Eng. 38, 371-383.

Yatim, H., Moszkowicz, P., Otterbein, M., Lang, P., 1993. Dynamic simulation of a batch extractive distillation process. Comput. Chem. Eng. 17, S57-S62.

Yi, C.C., Huang, W.C., Chien, I.L., 2018. Energy-efficient heterogeneous extractive distillation system for the separation of close-boiling cyclohexane/cyclohexene mixture. J. Taiwan Inst. Chem. Eng. 87, 26-35.

Yildirim, O., Kiss, A.A., Kenig, E.Y., 2011. Dividing wall columns in chemical process industry: a review on current activities. Sep. Purif. Technol. 80, 403-417.

You, X., Rodriguez-Donis, I., Gerbaud, V., 2014. Extractive distillation process optimisation of the 1.0-1a class system, acetone-methanol with water. Comput. Aided Chem. Eng. 33, 1315-1320.

You, X., Rodriguez-Donis, I., Gerbaud, V., 2015a. Improved design and efficiency of the extractive distillation process for acetone-methanol with water. Ind. Eng. Chem. Res. 54 (1), 491-501.

You, X., Rodriguez-Donis, I., Gerbaud, V., 2015b. Investigation of separation efficiency indicator for the optimization of the acetone-methanol extractive distillation with water. Ind. Eng. Chem. Res. 54 (43), 10863-10875.

You, X., Rodriguez-Donis, I., Gerbaud, V., 2016a. Low pressure design for reducing energy cost of extractive distillation for separating diisopropyl ether and isopropyl alcohol. Chem. Eng. Res. Des. 109, 540-552.

You, X., Rodriguez-Donis, I., Gerbaud, V., 2016b. Reducing process cost and $\mathrm{CO}_{2}$ emissions for extractive distillation by double-effect heat integration and mechanical heat pump. Appl. Energy 166, 128-140.

You, X., Gu, J., Peng, C., Rodriguez-Donis, I., Liu, H., 2017 a. Optimal design of extractive distillation for acetic acid dehydration with $\mathrm{N}$-methyl acetamide. Chem. Eng. Process.: Process Intensif. 120, 301-316.

You, X., Gu, J., Peng, C., Shen, W., Liu, H., 2017b. Improved design and optimization for separating azeotropes with heavy component as distillate through energy-saving extractive distillation by varying pressure. Ind. Eng. Chem. Res. 56 (32), 9156-9166.

You, X., Gu, J., Gerbaud, V., Peng, C., Liu, H., 2018. Optimization of pre-concentration, entrainer recycle and pressure selection for the extractive distillation of acetonitrile-water with ethylene glycol. Chem. Eng. Sci. 177, 354-368.

Yu, H., Ye, Q., Xu, H., Dai, X., Suo, X., Li, R., 2015. Comparison of alternative distillation processes for the maximum-boiling ethylenediamine dehydration system. Chem. Eng. Process.: Process Intensif. 97 (2015), 84-105.

Zhan, G.-X., Shen, B.-X., Sun, H., Chen, X., 2018. Extractive distillation approach to the removal of dimethyl disulfide from methyl tert-butyl ether: combined computational solvent screening and experimental process investigation. Ind. Eng. Chem. Res. 57 (9), 3348-3358.

Zhang, H., Ye, Q., Qin, J., Xu, H., Li, N., 2014. Design and control of extractive dividing-wall column for separating ethyl acetate-isopropyl alcohol mixture. Ind. Eng. Chem. Res. 53, 1189-1205.

Zhang, L., Zhang, Z., Shen, D., Lan, M., 2017. 2-Propanol dehydration via extractive distillation using a renewable glycerol-choline chloride deep eutectic solvent: vapor-liquid equilibrium. J. Chem. Eng. Data 62 (2), 872-877.

Zhang, X., Li, X., Li, G., Zhu, Z., Wang, Y., Xu, D., 2018a. Determination of an optimum entrainer for extractive distillation based on an isovolatility curve at different pressures. Sep. Purif. Technol. 201, 79-95.

Zhang, X., Zhao, Y., Wang, H., Qin, B., Zhu, Z., Zhang, N., Wang, Y., 2018b. Control of a ternary extractive distillation process with recycle splitting using a mixed entrainer. Ind. Eng. Chem. Res. 57 (1), 339-351.

Zhao, Y., Zhao, T., Jia, H., Li, X., Zhu, Z., Wang, Y., 2017a. Optimization of the composition of mixed entrainer for economic extractive distillation process in view of the separation of tetrahydrofuran/ethanol/water ternary azeotrope. J. Chem. Technol. Biotechnol. 92, 2433-2444.

Zhao, L., Lyu, X., Wang, W., Shan, J., Qiu, T., 2017b. Comparison of heterogeneous azeotropic distillation and extractive distillation methods for ternary azeotrope ethanol/toluene/water separation. Comput. Chem. Eng. 100, 27-37.

Zhao, Y., Ma, K., Bai, W., Du, D., Zhu, Z., Wang, Y., Gao, J., 2018. Energy-saving thermally coupled ternary extractive distillation process by combining with mixed entrainer for separating ternary mixture containing bioethanol. Energy 148, 296-308.

Zheng, H., Li, Y., Xu, C., 2017. Control of highly heat-integrated energy-efficient extractive distillation processes. Ind. Eng. Chem. Res. 56 (19), 5618-5635. 